\title{
Science Programs for a 2-m Class Telescope at Dome C, Antarctica: PILOT, the Pathfinder for an International Large Optical Telescope
}

\author{
M. G. Burton ${ }^{\mathrm{A}, \mathrm{M}}$, J. S. Lawrence ${ }^{\mathrm{A}}$, M. C. B. Ashley ${ }^{\mathrm{A}}$, J. A. Bailey ${ }^{\mathrm{B}, \mathrm{C}}$, C. Blake ${ }^{\mathrm{A}}$, \\ T. R. Bedding ${ }^{\mathrm{D}}$, J. Bland-Hawthorn ${ }^{\mathrm{B}}$, I. A. Bond ${ }^{\mathrm{E}}$, K. Glazebrook $^{\mathrm{F}}$, M. G. Hidas ${ }^{\mathrm{A}}$, \\ G. Lewis $^{\mathrm{D}}$, S. N. Longmore $^{\mathrm{A}}$, S. T. Maddison ${ }^{\mathrm{G}}$, S. Mattila ${ }^{\mathrm{H}}$, V. Minier ${ }^{\mathrm{I}}$, S. D. Ryder ${ }^{\mathrm{B}}$,

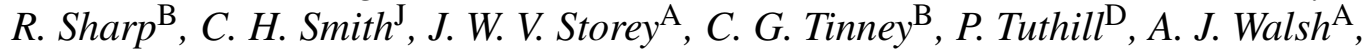

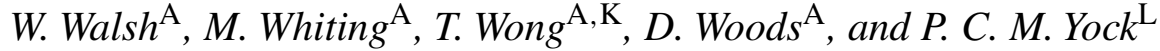 \\ A School of Physics, University of New South Wales, Sydney NSW 2052, Australia \\ ${ }^{B}$ Anglo Australian Observatory, Epping NSW 1710, Australia \\ ${ }^{C}$ Centre for Astrobiology, Macquarie University, Sydney NSW 2109, Australia \\ D University of Sydney, Sydney NSW 2006, Australia \\ E Massey University, Auckland, New Zealand \\ F John Hopkins University, Baltimore, MD 21218, USA \\ G Swinburne University, Melbourne VIC 3122, Australia \\ ${ }^{\mathrm{H}}$ Stockholm Observatory, Stockholm, Sweden \\ ${ }^{\text {I }}$ CEA Centre d'Etudes de Saclay, Paris, France \\ ${ }^{\mathrm{J}}$ Electro Optics Systems, Queanbeyan NSW 2620, Australia \\ ${ }^{\mathrm{K}}$ CSIRO Australia Telescope National Facility, Epping NSW 1710, Australia \\ ${ }^{\mathrm{L}}$ University of Auckland, Auckland, New Zealand \\ ${ }^{\mathrm{M}}$ Corresponding author. E-mail: M.Burton@unsw.edu.au
}

Received 2004 November 12, accepted 2005 April 12

\begin{abstract}
The cold, dry, and stable air above the summits of the Antarctic plateau provides the best groundbased observing conditions from optical to sub-millimetre wavelengths to be found on the Earth. Pathfinder for an International Large Optical Telescope (PILOT) is a proposed $2 \mathrm{~m}$ telescope, to be built at Dome C in Antarctica, able to exploit these conditions for conducting astronomy at optical and infrared wavelengths. While PILOT is intended as a pathfinder towards the construction of future grand-design facilities, it will also be able to undertake a range of fundamental science investigations in its own right. This paper provides the performance specifications for PILOT, including its instrumentation. It then describes the kinds of projects that it could best conduct. These range from planetary science to the search for other solar systems, from star formation within the Galaxy to the star formation history of the Universe, and from gravitational lensing caused by exo-planets to that produced by the cosmic web of dark matter. PILOT would be particularly powerful for wide-field imaging at infrared wavelengths, achieving near diffraction-limited performance with simple tip-tilt wavefront correction. PILOT would also be capable of near diffraction-limited performance in the optical wavebands, as well be able to open new wavebands for regular ground-based observation, in the mid-IR from 17 to $40 \mu \mathrm{m}$ and in the sub-millimetre at $200 \mu \mathrm{m}$.
\end{abstract}

Keywords: telescopes — site testing — atmospheric effects — techniques: high angular resolution — stars: formation - cosmology: observations

\section{Introduction - the Antarctic Plateau}

The highest regions of the Antarctic plateau provide a unique environment on the Earth for conducting observational astronomy. This is because of the extreme cold, the dryness, and the stability of the air column above these locations - leading to a lower sky background, greater transparency, and sharper imaging than at temperate sites.

The Antarctic high plateau includes an area about the size of Australia, all of which is above $3000 \mathrm{~m}$ elevation. With a year-round average temperature of $-50^{\circ} \mathrm{C}$, falling as low as $-90^{\circ} \mathrm{C}$ at times, the sky thermal emission, which dominates at wavelengths longer than $2.2 \mu \mathrm{m}$, is far less than at temperate sites (at shorter infrared wavelengths the emissivity is primarily due to $\mathrm{OH}$ airglow from the upper atmosphere). A reduced concentration of particulates in the atmosphere lowers the sky emissivity (predominantly arising from dust and aerosols at temperate sites), further lowering the background at these wavelengths. Columns of precipitable water vapour are less than $250 \mu \mathrm{m}$ for much of the year, opening atmospheric windows across the infrared and sub-millimetre bands. Wind speeds are low at the summits of the plateau, with violent storms 
non-existent. The thinness of the surface inversion layer, combined with the minimal turbulence above it, provides conditions of extraordinary stability, with the lowest levels of seeing on Earth. These conditions are also particularly suitable for wavefront correction. The plateau also has the lowest levels of seismic activity on the planet. Together with the low wind, this reduces constraints on the required strength and stiffness of large structures.

Taken together, these conditions create an important opportunity for observational astronomy, from the optical to the millimetre wavebands. Indeed, given the relative ease of access compared to space, they may provide the best environment from which to conduct some granddesign experiments, such as the search for exo-earths, for the next several decades. Nevertheless, the astronomy so far conducted in Antarctica has been largely confined to just a few of the competitive niches (see, for example, Indermuehle, Burton, \& Maddison 2005). These include a series of successful cosmic microwave background experiments and sub-millimetre astronomy with the modest aperture telescopes, together with, from particle astrophysics, the installation of networks of cosmic ray facilities, the building of the first neutrino telescope, and the collection of meteorites from blue-ice fields where they have been transported to after falling onto the plateau. Aside from site testing, no astronomy has yet been conducted from any of the summits of the Antarctic plateau. While there is no doubt that the performance of large Antarctic telescopes that operate in the optical and infrared would be significantly better than that of comparable facilities at temperate sites, so far the largest telescope to observe in these wavebands has been the $60 \mathrm{~cm}$ SPIREX telescope at the South Pole (Hereld 1994; Fowler et al. 1998). The South Pole, however, at $2835 \mathrm{~m}$, is on the flank of the plateau and suffers from the katabatic air flow off the summit at Dome A, which disturbs the seeing in the surface inversion layer. Better sites than South Pole are to be found on the summits of the plateau, in particular at the accessible site of Dome C. An intermediate-sized telescope at Dome $\mathrm{C}$ is an important next step in Antarctic astronomy, prior to investing in major optical/IR facilities. Its successful operation would demonstrate that the gains inferred from the site testing campaigns can in fact be realized. It would also allow the logistical and engineering requirements of running such a facility through the Antarctic winter to be appraised.

Operating such an intermediate-sized telescope as a technology demonstrator is only part of the requirement, however. The demands of scientific enquiry also mean that it is essential that such a telescope be able to undertake competitive science as well, even if its primary purpose is as a step towards more powerful facilities to follow. It is the purpose of this paper to consider the scientific case for such an intermediate facility - a 2-m class telescope capable of diffraction-limited imaging from optical wavebands to the mid-infrared (i.e. from 0.5 to $40 \mu \mathrm{m}$ ). We discuss below some of the scientific programs that such a facility, which we have dubbed PILOT — the Pathfinder for a International Large Optical Telescope - could tackle if it were built at Dome C. This $3250 \mathrm{~m}$ elevation site is the location of the new Concordia scientific station $\left(75^{\circ} \mathrm{S}, 123^{\circ} \mathrm{E}\right)$, built by the French and Italian national Antarctic programs (Candidi \& Lori 2003; Storey et al. 2003) and opened for winter operations in 2005. This document also builds on two earlier science cases for Antarctic astronomy, the first when the program was beginning in Australia (Burton et al. 1994), and the second when the emphasis was on building the 2-m Douglas Mawson Telescope, which focussed on wide-field thermal-IR imaging (Burton, Storey, \& Ashley 2001).

\section{The Advantages of Antarctica for Astronomy}

As a result of extensive site-testing programs that have been conducted at the South Pole for over two decades, and at Dome C since 1996, it has been established that there are a number of major advantages that an Antarctic plateau observatory would have over the same facility operating at temperate latitudes. These include:

- Low temperature: At wavelengths shortward of the blackbody-like peak in the sky-emission spectrum, the flux drops considerably for a small fall in temperature. Above the Antarctic plateau the background reduction relative to temperate sites, for a typical mid-winter temperature of $-60^{\circ} \mathrm{C}$, is $\sim 20$ times at near-infrared wavelengths $(2.2-5 \mu \mathrm{m})$ (Ashley et al. 1996; Nguyen et al. 1996; Phillips et al. 1999; Walden et al. 2005). This is equivalent to obtaining the same sensitivity using a telescope of several times the diameter at a temperate site (see Section 4.1.1). Between 2.27 and 2.45 $\mu \mathrm{m}$ the background drop is even greater, around 50 times.

- Low water vapour: With the precipitable water vapour content averaging $\sim 250 \mu \mathrm{m}$ above the plateau in winter (Chamberlin, Lane, \& Stark 1997; Lane 1998), the atmospheric transmission is considerably improved, particularly at mid-IR and sub-millimetre wavelengths (Chamberlain et al. 2000; Hidas et al. 2000; Calisse et al. 2004), over temperate locations. New windows become accessible for ground-based observation between 20 and $40 \mu \mathrm{m}$ and at $200 \mu \mathrm{m}$. In addition, the low water vapour also lowers the emissivity of the atmosphere, further reducing the sky flux. At the very highest location on the plateau, the $4200 \mathrm{~m}$ Dome A, the water vapour content may drop below $100 \mu \mathrm{m}$ at times, further opening new windows right across the far-infrared spectrum (see Lawrence 2004a).

- Low aerosol contribution: The lack of dust and other particulates in the atmosphere greatly reduces the contribution to sky emissivity from aerosols (Chamberlain et al. 2000; Walden et al. 2005), so further reducing the background emission in the mid-infrared bands (7-40 $\mu \mathrm{m})$.

The Line-By-Line Radiative Transfer Model (LBLRTM; Clough \& Iacono 1995) was used to model the atmospheric transmission and sky emission at Dome $\mathrm{C}$ across 


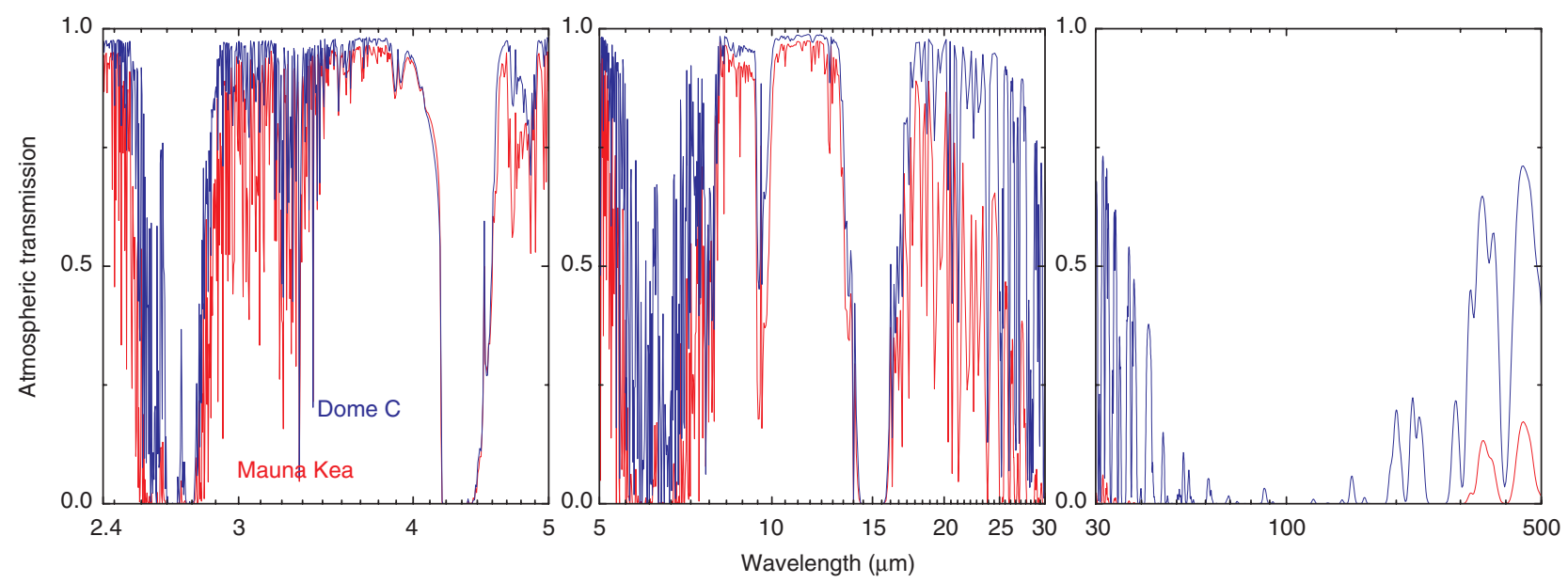

Figure 1 Atmospheric transmission calculated for Mauna Kea (red) and Dome C (blue) using the LBLRTM code, with the measured atmospheric parameters for the sites as input. The three panels show the start of the thermal-IR bands $(2.4-5.0 \mu \mathrm{m})$, the mid-IR $(5-30 \mu \mathrm{m})$ and the far-IR, and sub-millimetre bands $(30-500 \mu \mathrm{m})$, respectively.
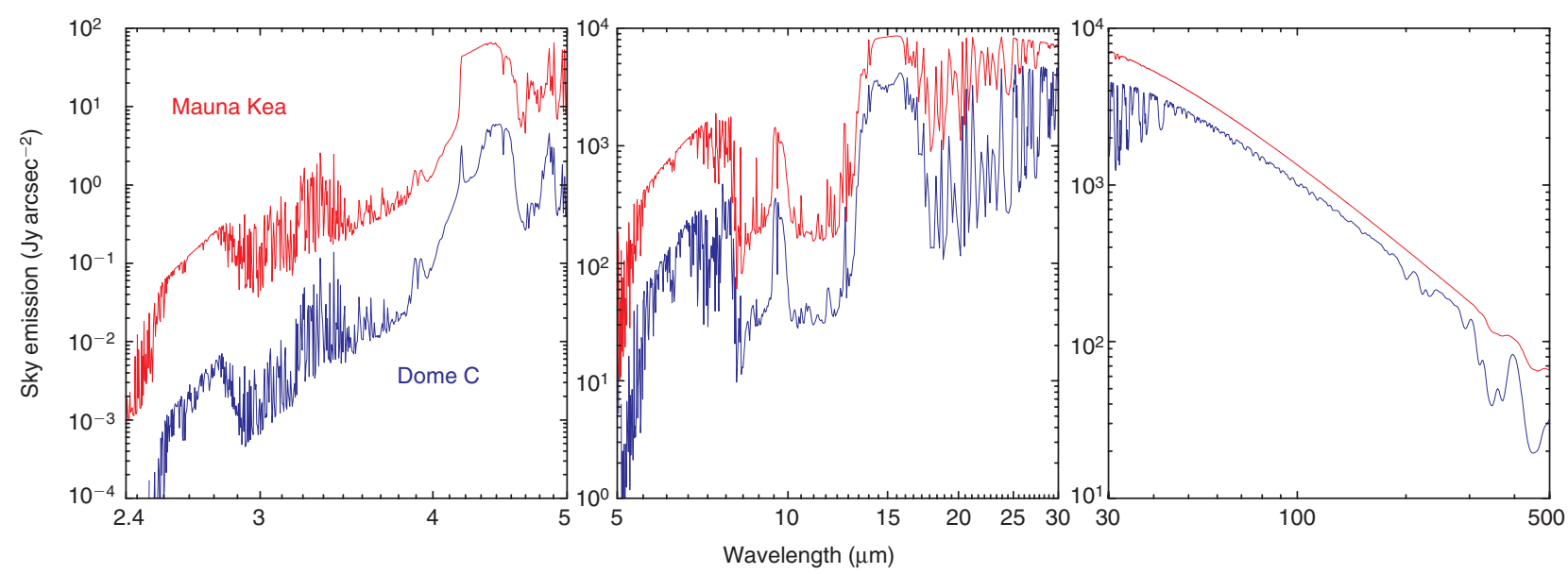

Figure 2 Atmospheric emission calculated for Mauna Kea (red) and Dome C (blue), using the LBLRTM code. The three panels show the start of the thermal-IR bands $(2.4-5.0 \mu \mathrm{m})$, the mid-IR $(5-30 \mu \mathrm{m})$, and the far-IR and sub-millimetre bands $(30-500 \mu \mathrm{m})$, respectively.

the infrared and sub-millimetre bands (see Lawrence 2004a for details). This code makes use of a model atmosphere whose input is the temperature, water vapour, pressure, and gaseous constituent profiles with height, based on data from comprehensive measurements made at the South Pole and from balloons launched at Dome C. Figures 1 and 2 show the results from this modelling, and compare the results calculated for Mauna Kea Observatory in Hawaii, generally regarded as the best temperate observatory site in the world. The background drop across the near- and mid-IR is clear, as are the new windows that open up in the mid-IR and sub-millimetre. The advantage is even greater towards the edges of the windows.

There are further advantages to be gained for the Antarctic plateau sites because of the nature of the atmospheric turbulence that causes astronomical seeing:

- Superb seeing: This results from the exceedingly stable air flow over the summits of the plateau. The major contribution to the wind on the plateau is katabatic in origin, and this implies calm conditions will exist on the summits of the plateau (Marks et al. 1996, 1999; Marks 2002; Travouillon et al. 2003a, 2003b). At Dome C, the average wind speed is just $2.8 \mathrm{~m} \mathrm{~s}^{-1}$, one of the lowest values of any observatory on Earth (Aristidi et al. 2005). The highest ever recorded wind speed there is just $20 \mathrm{~m} \mathrm{~s}^{-1}$. With the high-altitude jet stream rarely intruding, the remaining turbulence which causes astronomical seeing is driven by the wind-speed gradient within a narrow boundary layer, confined to no more than $30 \mathrm{~m}$ in height above Dome C. This leads to extraordinarily good seeing, with the mean value of $0.27^{\prime \prime}$ for the V-band determined from the first six weeks of winter-time data to be obtained from this site (Lawrence et al. 2004). This is less than half the value measured on Mauna Kea. For more than $25 \%$ of the time the seeing is below $0.15^{\prime \prime}$, values never attained from temperate locations. The daytime seeing is also very good, being less 
than $1^{\prime \prime}$ for much of the time (Aristidi et al. 2003), and falling as low as $0.2^{\prime \prime}$. Since the seeing varies as $\lambda^{-1 / 5}$, a 2-m telescope in Antarctica would have near diffractionlimited performance longward of the $\mathrm{H}$-band $(1.65 \mu \mathrm{m})$ in average seeing conditions.

- Wide isoplanatic angle: With small contributions to the seeing originating from the upper atmosphere, the isoplanatic angle is two to three times larger than at temperate sites $\left(\sim 6^{\prime \prime}\right.$ at V; Lawrence et al. 2004). This is because the angular size of turbulent cells, as seen by the telescope, is larger due to their proximity. The isoplanatic angle also depends on the Fried parameter $\left(r_{0}\right.$, the effective maximum aperture size for diffraction-limited performance). Since $r_{0}$ increases as $\lambda^{6 / 5}$, the isoplanatic angle can attain arcminute-sized values in the near-IR.

- Long coherence times: The slow drift of turbulent cells through the telescope field, resulting from the slow wind speed, serves to increase the coherence time to $\sim 8 \mathrm{~ms}$ at V-band (Lawrence et al. 2004). This is of particular importance when wavefront correction is being performed, as it means longer integration times and reduced adaptive optics (AO) system bandwidths. Combined with the larger isoplanatic angle, guide stars for wavefront correction will invariably be available for any source under study (see Marks et al. 1999). In addition, the coherence time also increases with the Fried parameter as $\lambda^{6 / 5}$, thus simplifying the operation of wavefront correction systems at longer wavelengths.

- Low scintillation noise: In addition to a wider isoplanatic angle, the shallower curvature on the wavefront of incident radiation reduces the scintillation noise. Since scintillation provides the principal limit to highprecision photometry, the reduced noise offers the prospect of more accurate measurement of the flux of stars, particularly when small variations are sought. The precision obtainable should be several times better than at temperate sites (Fossat 2003).

- Astrometric interferometry: The precision with which relative positions of objects can be determined over a narrow angle using an interferometer depends on $\int h^{2} C_{N}^{2}(h) \mathrm{d} h$, where $C_{N}^{2}(h)$ is the refractive index structure function at height $h$. Since these fluctuations are largely confined to the same narrow inversion layer as the previous four properties discussed above, rather than arising from a high-altitude jet stream, this leads to large gains in the precision of measurement of source positions. Calculations by Lloyd, Oppenheimer, \& Graham (2002) suggest that a given accuracy could be obtained 300 times faster at the South Pole than from a temperate site, with precisions of a few micro-arcseconds accuracy attainable.

- Continuous observation: At the South Pole any source above the horizon can be observed continuously, weather permitting, providing the opportunity for long time-series measurements. In addition, at thermal infrared wavelengths (i.e. $\lambda>3 \mu \mathrm{m}$ ) daytime observations can readily be undertaken, still with reduced backgrounds compared to temperate sites at night time. At Dome $\mathrm{C}$, while there can be up to a $30^{\circ}$ variation in elevation during the course of the day, sources generally remain accessible for longer time periods than at temperate sites.

- Stability: While limited data currently exist on the sky stability at Dome C (aside from the turbulence discussed above), indications are that the sky conditions are very stable. For instance, measurements with an all-sky camera in 2001 showed clear skies for $74 \%$ of the time during winter (Ashley et al. 2005). The infrared sky during a period of clear sky in 2004 January (Walden et al. 2005) showed only a $10 \%$ variation in flux at $11 \mu \mathrm{m}$ over five days, an exceptionally stable value compared to the variations experienced at temperate sites.

- Costs: Increased costs are not a major factor in Antarctic operations, a facet which often comes as a surprise for scientists new to the field. While some costs are higher than at temperate sites, others are far lower. For instance, the need for a protective dome are minimal in the absence of storms and strong winds. The smaller instrument and telescope sizes required, for the same performance as a temperate site, also reduces the cost. While logistics considerations determine where a telescope may be placed (i.e. at an existing base), they then make transportation relatively simple - Antarctica provides the only observatory sites in the world adjacent to airports with heavy-lift capability, for example. Other cost gains are more indirect, but significant nevertheless. For instance, there are no indigenous species at the site whose habitat may be disturbed.

\section{The Disadvantages of Antarctica for Astronomy}

There are, of course, certain disadvantages to working in Antarctica. The most notable is that the telescope requires winter fastness. Repairing a telescope during mid-winter is particularly difficult. Among the practical difficulties of installation and operation, the dry air increases the risk of static damage to electronic components. The use of liquid helium over the winter months is a major problem because supply lines must be maintained across inter-continental distances. For nine months of the year the site will be physically isolated from the rest of the world. See Ashley et al. (2004) for further discussion of these issues.

The South Pole, lying under the auroral circle, suffers from frequent aurorae. Dome $\mathrm{C}$, however, lies close to the geomagnetic South Pole, so that that aurorae are generally below the horizon. Auroral emission is largely concentrated in a few spectral lines, particularly atomic oxygen and bands from molecular oxygen and nitrogen. Auroral emission has not yet been measured at Dome C, but based on extrapolation from the South Pole, is estimated to have median values in B-band of between 22.9 and 24.0 mag $\operatorname{arcsec}^{-2}$, and in V-band between 23.7 and $24.7 \mathrm{mag} \mathrm{arcsec}^{-2}$ (Dempsey et al. 2005). These estimates are about three magnitudes fainter than at the South Pole, 
and less than the anticipated night sky emission in these bands at Dome C (22 mag $\operatorname{arcsec}^{-2}$ at V; see Table $\left.1^{1}\right)$.

The amount of astronomical dark time (when the Sun is more than $18^{\circ}$ below the horizon, in the absence of the Moon) at Dome $\mathrm{C}$ is only $50 \%$ of that at temperate sites. Fortunately this has no affect on observations beyond $1 \mu \mathrm{m}$. There is also less of the sky accessible for viewing - the converse of being able to view other parts of the sky for longer periods. However, several important targets are well placed; the Magellanic Clouds pass overhead and the Galactic Centre is readily accessible, for instance.

Perhaps the biggest issue to contend with regarding working on the Antarctic plateau is that of human psychology. Spending a winter on the plateau, in several months of continuous darkness, is a challenging experience, and there are relatively few skilled people who would consider doing this. However, with nearly five decades of such experiences from the Amundsen-Scott South Pole station, it is clear that the challenges can be met. A harder challenge may be that of human perceptions of the continent. Most people's knowledge of Antarctica is based on stories from the 'heroic age' of Antarctic exploration in the early 20th century, where events were shaped by the adverse weather conditions faced on the coastal fringes of the continent. The climate in the interior is vastly different, as discussed in Section 2, and modern technology now allows humans to work there in comfort.

\section{PILOT - the Pathfinder for an International Large Optical Telescope}

PILOT is envisaged as a 2-m class telescope, to be sited at Dome C, Antarctica. It would have an alt-azimuth mount, with an $f / 1.5$ primary figured to a wavefront error below $40 \mathrm{~nm}^{2}$. There would be a Cassegrain focus and two Gregorian-fed Nasmyth focii with an $f / 20$ beam, providing an unvignetted field of view of at least one degree. One of these focii would be capable of feeding the beam to a future multi-telescope interferometer. A 45-element adaptive secondary would be used for wavefront correction.

\subsection{Performance Specifications}

\subsubsection{Sensitivity}

The low-infrared background and good seeing at Dome $\mathrm{C}$ implies that a telescope placed there should be an order of magnitude more sensitive than a mid-latitude telescope of the same size for many kinds of observations. In this section we quantify this statement by making a detailed examination of the performance that PILOT would have, in comparison to current 8-m class telescopes on the best temperate sites, as well as to possible future 8-m telescopes in Antarctica.

\footnotetext{
${ }^{1}$ Note that the standard notation $2(-7) \equiv 2 \times 10^{-7}$, etc. is used in all tables in this paper.

2 This high precision, better than normally demanded of optical telescopes, is required to reach the diffraction-limited image quality $\left(0.05^{\prime \prime}\right.$ resolution at $550 \mathrm{~nm}$ ) that the site makes possible.
}

In comparing the relative sensitivity of different-sized telescopes, we must take account of whether point sources or extended objects are being measured, as well as the waveband of interest for a particular study. The comparison also depends on the capabilities of any adaptive optics systems being used. We have thus calculated sensitivities for both point and extended sources and show these in Figure 3 and Tables 1 and 2. We compare these to the values for an 8-m telescope on Mauna Kea, as well as to an $8-\mathrm{m}$ telescope built at Dome $\mathrm{C}$, for the same instrument parameters. The figure shows the limiting magnitudes for SNR $=10$ in an hour of observation in the principal filter bands, for background limited operation. Seeing-limited spatial resolution (or diffraction-limited, if that is larger), as indicated in Table 1, is assumed (see also Figure 4).

At short wavelengths, telescope aperture is the most important parameter determining sensitivity, and this is reflected in the performance figures. When the thermal background dominates; however, the sensitivity of an Antarctic $2 \mathrm{~m}$ is similar to that of a temperate latitude $8 \mathrm{~m}$. An Antarctic $8 \mathrm{~m}$ is typically an order of magnitude more sensitive than a temperate latitude $8 \mathrm{~m}$ in all observing bands. In the thermal infrared $(\lambda>3 \mu \mathrm{m})$, the diffraction limit exceeds the seeing, and so the best spatial resolution is then achieved with the larger facility. Again, an Antarctic $8 \mathrm{~m}$ would have superior performance in all wavebands.

Because of the superb seeing, the spatial resolution attained with a 2-m Antarctic telescope is superior to the temperate $8 \mathrm{~m}$, for all wavelengths less than $2.3 \mu \mathrm{m}$. A gain of a factor of two in resolution is typical in the optical bands.

The sensitivity calculations in Table 1 do not take into account of the gains possible through the further use of a tip-tilt or an AO system. This serves to concentrate the flux into a smaller angular distribution, so improving the sensitivity for background limited operation. As discussed in Section 4.1.2, an AO system could achieve a Strehl ratio as high as 0.8 in V-band at Dome C, whereas only seeinglimited resolution is possible in this band from Mauna Kea. This can lead to another gain of up to $\sim 2$ mag in the point source sensitivity at $\mathrm{V}$ for PILOT, though this would only apply within the isoplanatic angle (of $\sim 6^{\prime \prime}$ at V).

Finally, it should be noted that the raw sensitivity figures do not demonstrate the full gain attainable in mid-IR wavelengths, since the background flux is considerably more stable in Antarctica as well. Fluctuations in the background provide the primary limitation to photometry at temperate sites. These calculations also do not show the greater wavelength range where observations are possible in Antarctica - the result of the opening of new windows because of the drier atmosphere.

\subsubsection{Spatial Resolution and Isoplanatic Angle}

As discussed in Section 2, the data obtained so far suggest that the median seeing at Dome $\mathrm{C}$ is more than a factor of two better than at the best temperate sites. In natural 
Table 1. Calculated Point Source Sensitivities

\begin{tabular}{|c|c|c|c|c|c|c|c|c|c|c|}
\hline \multirow[t]{2}{*}{ Band } & \multirow{2}{*}{$\begin{array}{c}\lambda \\
(\mu \mathrm{m})\end{array}$} & \multirow{2}{*}{$\begin{array}{c}\Delta \lambda \\
(\mu \mathrm{m})\end{array}$} & \multicolumn{3}{|c|}{ Telescope } & \multicolumn{3}{|c|}{ Spatial resolution } & \multicolumn{2}{|c|}{ Background } \\
\hline & & & Mauna Kea 8 m & $\begin{array}{l}\text { Antarctic } 2 \mathrm{~m} \\
\text { (PILOT) }\end{array}$ & Antarctic $8 \mathrm{~m}$ & Mauna Kea 8 m & $\begin{array}{l}\text { Antarctic } 2 \mathrm{~m} \\
(\operatorname{arcsec})\end{array}$ & Antarctic $8 \mathrm{~m}$ & $\begin{array}{l}\text { Mauna Kea } \\
\text { (Jy arcse }\end{array}$ & $\begin{array}{l}\text { Antarctic } \\
\left.\mathrm{ec}^{-2}\right)\end{array}$ \\
\hline V & 0.55 & 0.09 & $\begin{array}{l}26.3 \\
1(-7)\end{array}$ & $\begin{array}{l}25.5 \\
2(-7)\end{array}$ & $\begin{array}{l}27.1 \\
5(-8)\end{array}$ & 0.50 & 0.25 & 0.24 & $6(-6)$ & $6(-6)$ \\
\hline $\mathrm{R}$ & 0.65 & 0.15 & $\begin{array}{l}25.9 \\
1(-7)\end{array}$ & $\begin{array}{l}25.1 \\
3(-7)\end{array}$ & $\begin{array}{l}26.7 \\
6(-8)\end{array}$ & 0.48 & 0.24 & 0.23 & $1(-5)$ & $1(-5)$ \\
\hline I & 0.80 & 0.15 & $\begin{array}{l}25.1 \\
2(-7)\end{array}$ & $\begin{array}{l}24.3 \\
4(-7)\end{array}$ & $\begin{array}{l}25.9 \\
9(-8)\end{array}$ & 0.46 & 0.24 & 0.23 & $2(-5)$ & $2(-5)$ \\
\hline $\mathrm{J}$ & 1.21 & 0.26 & $\begin{array}{l}23.0 \\
1(-6)\end{array}$ & $\begin{array}{l}22.5 \\
2(-6)\end{array}$ & $\begin{array}{l}24.1 \\
4(-7)\end{array}$ & 0.42 & 0.24 & 0.21 & $9(-4)$ & $5(-4)$ \\
\hline $\mathrm{H}$ & 1.65 & 0.29 & $\begin{array}{l}21.8 \\
2(-6)\end{array}$ & $\begin{array}{l}21.4 \\
3(-6)\end{array}$ & $\begin{array}{l}23.2 \\
6(-7)\end{array}$ & 0.40 & 0.26 & 0.20 & $3(-3)$ & $1(-3)$ \\
\hline $\mathrm{K}$ & $\begin{array}{l}2.16 \\
2.30\end{array}$ & $\begin{array}{l}0.22 \\
0.23\end{array}$ & $\begin{array}{l}21.2 \\
2(-6)\end{array}$ & $\begin{array}{l}21.3 \\
2(-6)\end{array}$ & $\begin{array}{l}23.3 \\
3(-7)\end{array}$ & 0.38 & 0.30 & 0.19 & $2(-3)$ & $1(-4)$ \\
\hline $\mathrm{L}$ & 3.76 & 0.65 & $\begin{array}{l}16.7 \\
5(-5)\end{array}$ & $\begin{array}{l}16.3 \\
8(-5)\end{array}$ & $\begin{array}{l}18.6 \\
9(-6)\end{array}$ & 0.35 & 0.42 & 0.19 & $2(+0)$ & $2(-1)$ \\
\hline M & 4.66 & 0.24 & $\begin{array}{l}14.4 \\
3(-4)\end{array}$ & $\begin{array}{l}14.9 \\
2(-4)\end{array}$ & $\begin{array}{l}17.4 \\
2(-5)\end{array}$ & 0.34 & 0.52 & 0.20 & $4(+1)$ & $5(-1)$ \\
\hline $\mathrm{N}$ & 11.5 & 1.0 & $\begin{array}{l}10.7 \\
2(-3)\end{array}$ & $\begin{array}{l}9.1 \\
8(-3)\end{array}$ & $\begin{array}{l}12.0 \\
6(-4)\end{array}$ & 0.40 & 1.2 & 0.32 & $2(+2)$ & $2(+1)$ \\
\hline Q & 20 & 1.0 & $\begin{array}{l}7.2 \\
4(-2)\end{array}$ & $\begin{array}{l}5.5 \\
2(-1)\end{array}$ & $\begin{array}{l}8.5 \\
1(-2)\end{array}$ & 0.57 & 2.1 & 0.53 & $3(+3)$ & $5(+2)$ \\
\hline
\end{tabular}

Comparison of point source sensitivities in Vega-magnitudes (top line of pair) and Janskys (bottom line of pair) for an 8-m telescope on Mauna Kea (e.g. Gemini), a 2-m telescope on Dome C (i.e. PILOT), and an 8-m telescope at Dome C, as a function of waveband (listed are central wavelengths and bandpasses; for $\mathrm{K}$ band different choices are provided applicable to Mauna Kea and Dome C).

The SNR = 10 after one hour of integration, for the spatial resolution listed in the relevant columns. This is calculated by adding, in quadrature, the seeing to the diffraction limit, for the relevant combination of site and telescope parameters (see also Figure 4).

For the background determination, the pixel scale is taken to be half this value, with the object flux assumed to be summed over 25 such pixels. The telescope emissivity has been taken as $3 \%$ with a temperature of $0^{\circ} \mathrm{C}$ at Mauna Kea and $-60^{\circ} \mathrm{C}$ at Dome C. An overall system efficiency of $50 \%$ is assumed in all cases, together with the spectral resolution as listed for each bandpass. The sky fluxes, in Jy arcsec ${ }^{-2}$, adopted for each site are listed in the last two columns.

Further details are provided in Lawrence (2004a). These calculations have not taken into account the further gains possible when an AO system is operating. This is discussed further in Section 4.1.2.
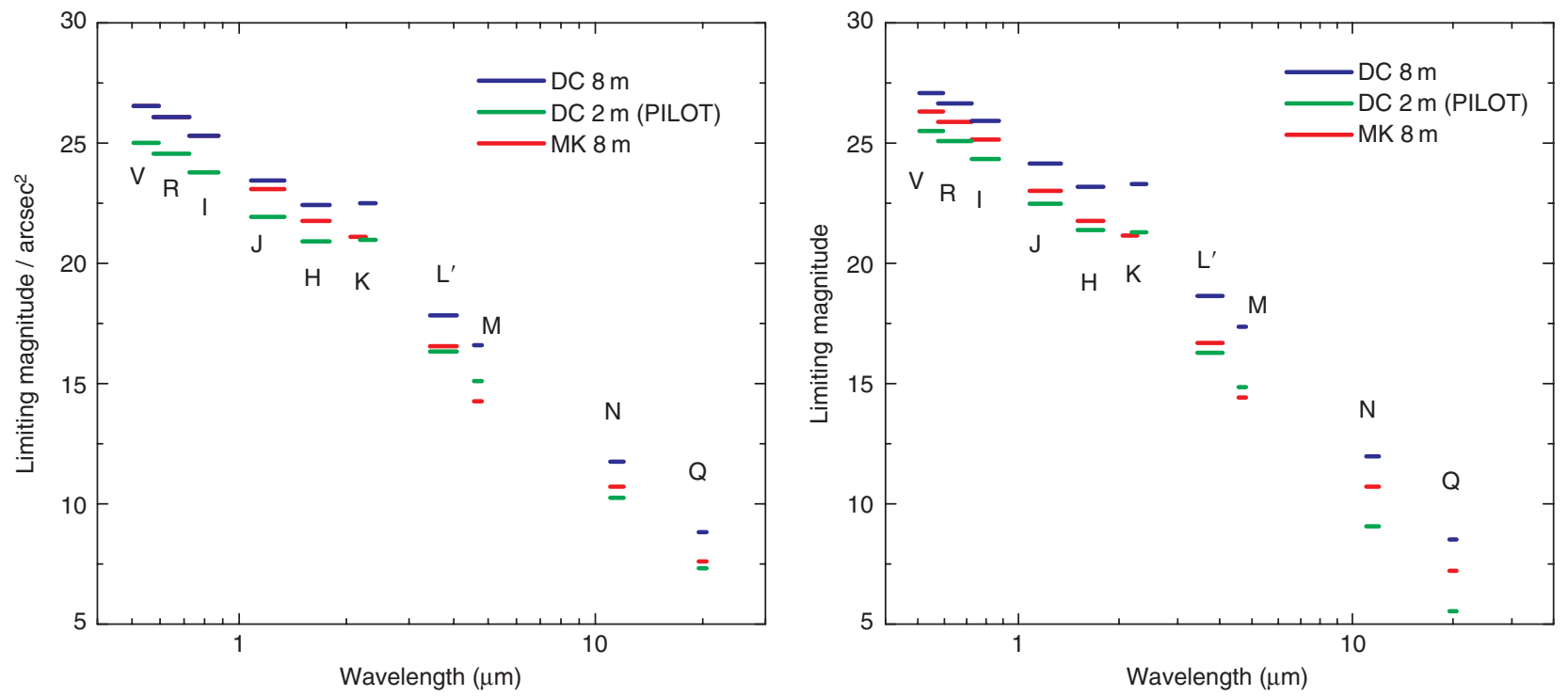

Figure 3 Comparison of extended source (left) and point source sensitivities (right) (in magnitudes per square arcsecond and magnitudes, respectively) for an 8-m telescope on Mauna Kea (red), a 2-m telescope on Dome C (i.e. PILOT; green), and an 8-m telescope at Dome C (blue), as a function of waveband, for a SNR $=10$ after one hour of integration. The width of each bar indicates the filter bandpass adopted. Further details regarding the assumptions made in these calculations are given in Table 1. 
Table 2. Calculated Extended Source Sensitivities

\begin{tabular}{|c|c|c|c|c|c|}
\hline \multirow[t]{2}{*}{ Band } & \multirow[t]{2}{*}{$\lambda(\mu \mathrm{m})$} & \multirow[t]{2}{*}{ Width $(\mu \mathrm{m})$} & \multicolumn{3}{|c|}{ Telescope } \\
\hline & & & Mauna Kea 8 m & $\begin{array}{l}\text { Antarctic } 2 \mathrm{~m} \\
\text { (PILOT) }\end{array}$ & Antarctic $8 \mathrm{~m}$ \\
\hline V & 0.55 & 0.09 & $\begin{array}{l}26.5 \\
9(-8)\end{array}$ & $\begin{array}{l}25.0 \\
4(-7)\end{array}$ & $\begin{array}{l}26.5 \\
9(-8)\end{array}$ \\
\hline $\mathrm{R}$ & 0.65 & 0.15 & $\begin{array}{l}26.1 \\
1(-7)\end{array}$ & $\begin{array}{l}24.6 \\
4(-7)\end{array}$ & $\begin{array}{l}26.1 \\
1(-7)\end{array}$ \\
\hline I & 0.80 & 0.15 & $\begin{array}{l}25.3 \\
2(-7)\end{array}$ & $\begin{array}{l}23.8 \\
7(-7)\end{array}$ & $\begin{array}{l}25.3 \\
2(-7)\end{array}$ \\
\hline $\mathrm{J}$ & 1.21 & 0.26 & $\begin{array}{l}23.1 \\
1(-6)\end{array}$ & $\begin{array}{l}21.9 \\
3(-6)\end{array}$ & $\begin{array}{l}23.4 \\
7(-7)\end{array}$ \\
\hline $\mathrm{H}$ & 1.65 & 0.29 & $\begin{array}{l}21.8 \\
2(-6)\end{array}$ & $\begin{array}{l}20.9 \\
5(-6)\end{array}$ & $\begin{array}{l}22.4 \\
1(-6)\end{array}$ \\
\hline K & $\begin{array}{l}2.16 \\
2.30\end{array}$ & $\begin{array}{l}0.22 \\
0.23\end{array}$ & $\begin{array}{l}21.1 \\
2(-6)\end{array}$ & $\begin{array}{l}21.0 \\
3(-6)\end{array}$ & $\begin{array}{l}22.5 \\
6(-7)\end{array}$ \\
\hline $\mathrm{L}$ & 3.76 & 0.65 & $\begin{array}{l}16.6 \\
6(-5)\end{array}$ & $\begin{array}{l}16.3 \\
7(-5)\end{array}$ & $\begin{array}{l}17.8 \\
2(-5)\end{array}$ \\
\hline M & 4.66 & 0.24 & $\begin{array}{l}14.3 \\
3(-4)\end{array}$ & $\begin{array}{l}15.1 \\
1(-4)\end{array}$ & $\begin{array}{l}16.6 \\
3(-5)\end{array}$ \\
\hline $\mathrm{N}$ & 11.5 & 1.0 & $\begin{array}{l}10.7 \\
2(-3)\end{array}$ & $\begin{array}{l}10.2 \\
3(-3)\end{array}$ & $\begin{array}{l}11.8 \\
7(-4)\end{array}$ \\
\hline Q & 20 & 1.0 & $\begin{array}{l}7.6 \\
3(-2)\end{array}$ & $\begin{array}{l}7.3 \\
4(-2)\end{array}$ & $\begin{array}{l}8.8 \\
9(-3)\end{array}$ \\
\hline
\end{tabular}

Comparison of extended source sensitivities in Vega-magnitudes per square arcsec (top line of pair) and Janskys per square arcsec (bottom line of pair), for an 8-m telescope on Mauna Kea, a 2-m telescope on Dome C (i.e. PILOT) and an 8-m telescope at Dome $\mathrm{C}$, as a function of waveband. The SNR $=10$ after one hour of integration. Other details are as for Table 1.

seeing conditions, without the application of any wavefront correction, a 2-m telescope can therefore achieve better resolution in the visible and near-IR than much larger facilities at temperate sites. Of course, since the seeing improves with wavelength as $\lambda^{-0.2}$, eventually the larger facility will provide superior resolution on account of its smaller diffraction limit. Figure 4 illustrates the seeing-limited resolution attainable at Dome $\mathrm{C}$ and Mauna Kea as a function of wavelength for 2-m and 8-m sized telescopes. For the best quartile conditions, near diffractionlimited performance for a 2-m telescope at Dome $\mathrm{C}$ is obtained in H-band (and longer wavelengths), whereas in median seeing conditions this occurs in K-band. An 8-m telescope on Mauna Kea, however, has superior resolution to a 2- $\mathrm{m}$ telescope at Dome $\mathrm{C}$ for wavelengths greater than $3 \mu \mathrm{m}$ (L-band), for then the diffraction limit exceeds the seeing for the 2-m telescope.

The turbulence profile above Dome $\mathrm{C}$ is unique, being largely confined to a layer close to the surface. This, combined with a lack of high altitude winds, results in significant improvements for the performance of any wavefront correction system compared to a typical midlatitude site. Based on the measurements of the seeing and turbulence profile at Dome $\mathrm{C}$, as measured with a SODAR and MASS (Lawrence et al. 2004), we have calculated in Figure 5 what the Strehl ratio (the ratio of the peak flux to that obtained with diffraction-limited performance) would be for a tip-tilt system on the PILOT telescope (see Lawrence 2004b for further details).

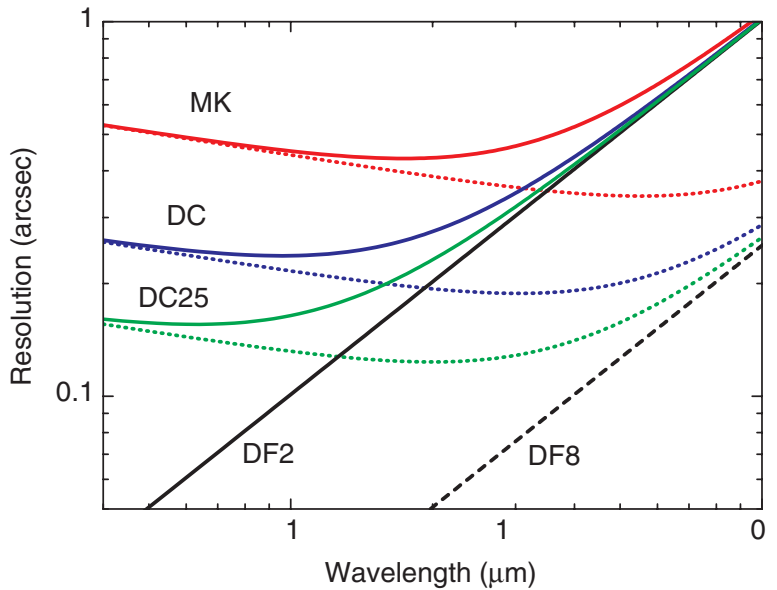

Figure 4 Resolution attainable with a 2-m telescopes (solid lines) and an 8-m telescope (dotted lines) at both Dome C and Mauna Kea, as a function of wavelength. The resolution is calculated by adding, in quadrature, the seeing to the diffraction limit. Curves are shown for Mauna Kea (red), median seeing conditions at Dome C (blue) and for the best $25 \%$ conditions at Dome C (green). Also shown in black, for comparison, is the diffraction-limited performance for a 2-m and an 8-m telescopes.

From these figures it can be seen that at Dome C tiptilt correction recovers most of the diffraction limit in the $\mathrm{J}-, \mathrm{H}-$, \& K-bands (i.e. 1.2-2.4 $\mu \mathrm{m}$ ), even in median seeing conditions. This occurs over a wide field of view of several arcmin (the tilt isoplanatic angle). It can also be achieved 

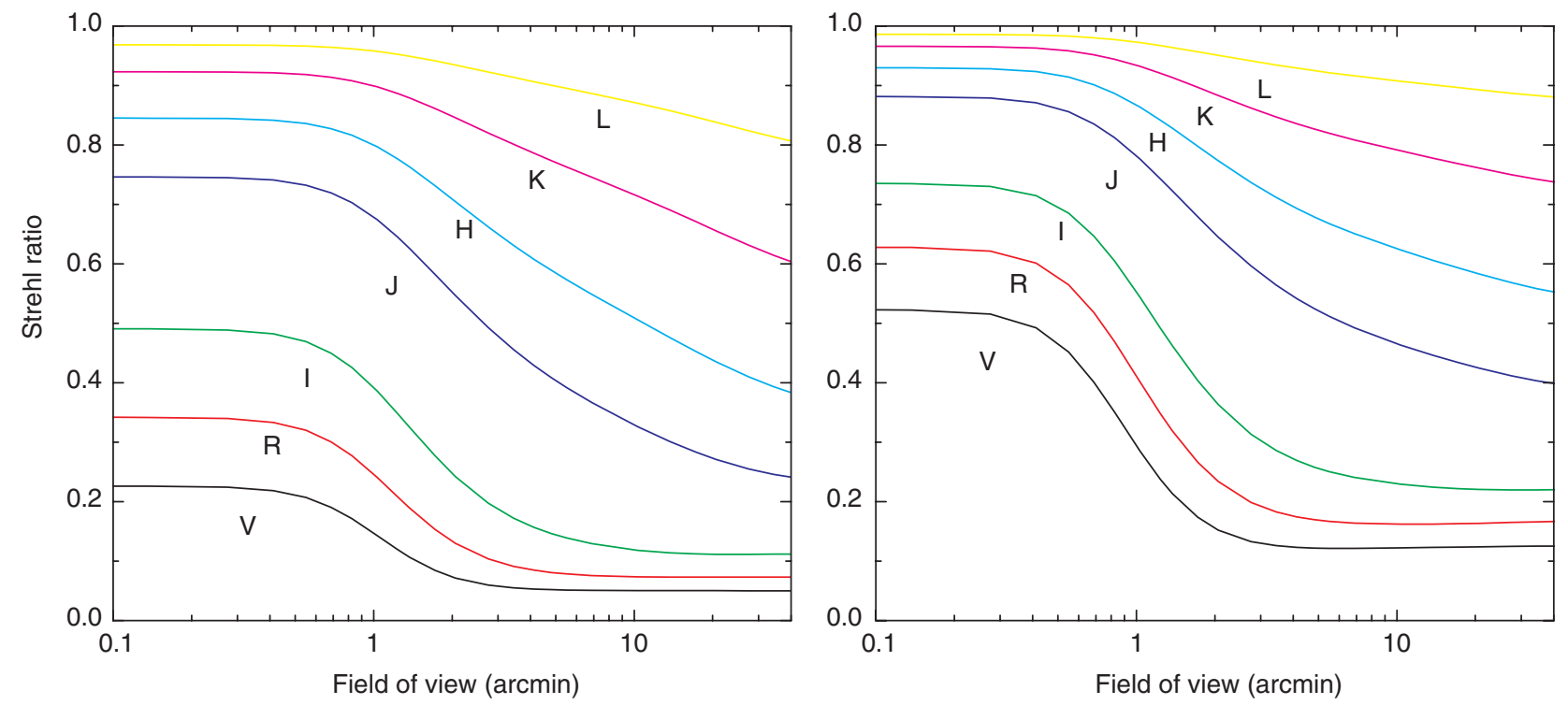

Figure 5 Strehl ratio (the ratio of the peak flux to the flux obtained with diffraction limited imaging), as a function of angular distance from the field centre, for a tip-tilt correction system operating on a 2-m telescope at Dome C, for wavebands from optical (V) to the thermal-IR (L). The left-hand plot shows the values in the median seeing conditions, the right-hand plot in the best $25 \%$ seeing conditions. For longer wavebands (M, N, Q), the Strehl ratio is effectively unity over all feasible fields of view. The right-hand axes of these plots reflect the Strehl ratio obtained in the natural seeing conditions.

over the entire sky, as there will always be a star available for wavefront correction within the tilt isoplanatic angle.

Using a higher order AO system than tip-tilt will, of course, provide superior angular resolution (within the isoplanatic angle) on a temperate 8-m telescope than with PILOT. In the J-band $(1.25 \mu \mathrm{m})$, for instance, this is equivalent to having a guide star bright enough to be used for AO correction within $\sim 10^{\prime \prime}$ of the source on Mauna Kea. However, no correction can be made for larger angles, resulting in seeing-limited performance over larger fields of view on Mauna Kea. In addition, only a small proportion of the sky can be covered with such a system as it relies on the existence of the nearby guide star.

AO systems provide the best spatial resolution possible for a telescope, albeit over much smaller fields of view than a tip-tilt system. The performance of an $\mathrm{AO}$ system is a function of many parameters, including the number of actuators in use, the size of the telescope, the brightness of the guide star being used for correction, and its angular distance from the object under study, in addition to the site seeing and isoplanatic angle. In Figure 6 we compare the Strehl ratio obtainable with an on-axis AO system with 45 actuators on a 2-m telescope at Dome C to that of the same-sized telescope on Mauna Kea (see Lawrence 2004b for details). The Strehl ratio is shown as a function of the magnitude of the star being used for the correction; i.e. within the isoplanatic angle, which is 6 " at $\mathrm{V}$ for Dome $\mathrm{C}$ and $2^{\prime \prime}$ at Mauna Kea. As can be seen, significant correction would be possible in the visible when guide stars brighter than 10th magnitude exist within 6 " of a source at Dome C. In contrast, from Mauna Kea a star brighter than 8th magnitude, less than $2^{\prime \prime}$ from the source,

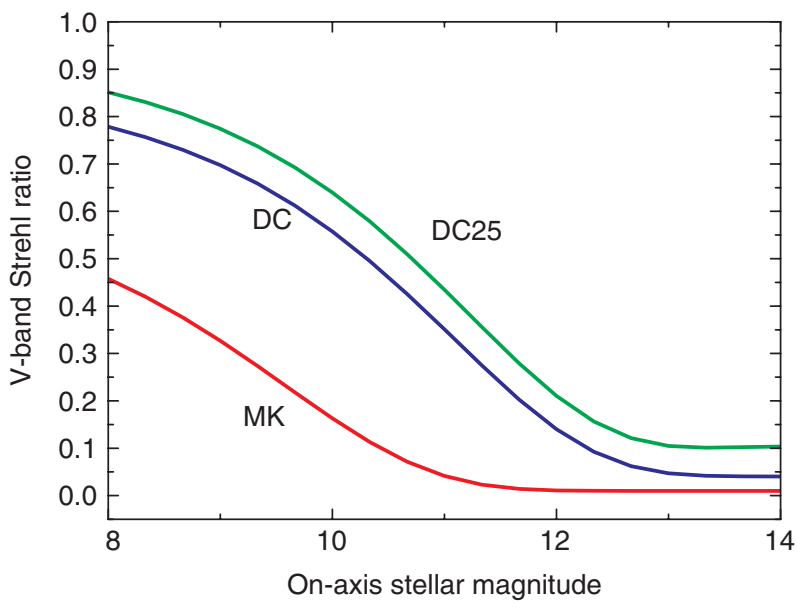

Figure 6 Strehl ratio obtained in the visible (V-band), as a function of the magnitude of an on-axis guide star used for AO correction, for 2-m aperture telescopes operating with 45 actuators at Dome C and at Mauna Kea. For Dome C, the performance is shown for both the median and best $25 \%$ seeing conditions, whereas for Mauna Kea it is for the median seeing. In practice, the guide star must also be found within the isoplanatic angle, which is 6" in V-band at Dome C, about nine times larger in area than at Mauna Kea.

is required for correction to be possible. This tight constraint effectively makes AO systems unusable in optical wavebands at Mauna Kea, whereas they become feasible at Dome C. Furthermore, with a ground layer AO (GLAO) system at Dome C, correction should be obtainable in the visible over a wide field of view (albeit with a slightly lower Strehl ratio; e.g. see Ragazzoni 2004) ${ }^{3}$.

\footnotetext{
${ }^{3}$ With PILOT in a Gregorian configuration, an adaptive secondary conjugates to an atmospheric layer some $20 \mathrm{~m}$ above the telescope. Such a
} 
Table 3. Strawman Instrument Suite for PILOT

\begin{tabular}{lcclccc}
\hline Wavebands & $\begin{array}{c}\text { Wavelength } \\
\text { (microns) }\end{array}$ & $\begin{array}{c}\text { Array } \\
\text { size }\end{array}$ & $\begin{array}{c}\text { Detector } \\
\text { material }\end{array}$ & $\begin{array}{c}\text { Pixel scale } \\
(\operatorname{arcsec})\end{array}$ & $\begin{array}{c}\text { Field of view } \\
(\operatorname{arcmin})\end{array}$ & $\begin{array}{c}\text { Figure of merit } \\
A \Omega / \Delta \Omega\left(\mathrm{m}^{2}\right)\end{array}$ \\
\hline Optical: VRI (High resolution) & $0.5-1.0$ & $4 \mathrm{~K}$ & $\mathrm{Si}$ & $0.03^{\prime \prime}$ & $2.0^{\prime}$ & $1(+7)$ \\
Optical: VRI (Wide field) & $0.5-1.0$ & $4 \mathrm{~K}$ & $\mathrm{Si}$ & $0.1^{\prime \prime}$ & $6.8^{\prime}$ & $1(+7)$ \\
Near-IR: JHK (High resolution) & $1.2-2.4$ & $4 \mathrm{~K}$ & $\mathrm{HgCdTe}$ & $0.08^{\prime \prime}$ & $5.3^{\prime}$ & $1(+7)$ \\
Near-IR: JHK (Wide field) & $1.2-2.4$ & $4 \mathrm{~K}$ & $\mathrm{HgCdTe}$ & $0.30^{\prime \prime}$ & $20.5^{\prime}$ & $1(+7)$ \\
Thermal-IR: KLM & $2.2-5.9$ & $1 \mathrm{~K}$ & $\mathrm{InSb}$ & $0.23^{\prime \prime}$ & $4^{\prime}$ & $8(+5)$ \\
Mid-IR: NQ & $7-40$ & $0.5 \mathrm{~K}$ & $\mathrm{SiAs}$ & $0.7^{\prime \prime}$ & $6^{\prime}$ & $2(+5)$ \\
Sub-mm & $200-450$ & $1 \mathrm{~K}$ & $\mathrm{NbN}$ & $25-60^{\prime \prime}$ & $0.5-1.0^{\prime}$ & $8(-1)$ \\
\hline
\end{tabular}

Outline specifications for seven instrument concepts that could take advantage of the capabilities offered by PILOT. Aside from the wide-field optical and near-IR imagers, all cameras sample at half the diffraction limit — to take advantage of the good natural seeing (the wide-field optical camera samples at half the seeing limit). Their fields of view are then determined by the largest, commercially available array for the relevant waveband. Larger fields would be possible by butting several such arrays together. While the performance has been calculated for broadband filters, a suite of narrowband filters ( $1 \%$ spectral resolution) would also be included in any instrument. If a multi-beam instrument was built for the sub-millimetre, rather than a single-element bolometer, this would of course greatly increase the capabilities in this waveband too. The Figure of Merit is determined by $A \Omega / \Delta \Omega$, where $A$ is the telescope aperture ( $2 \mathrm{~m}$ diameter), $\Omega$ the field of view of the camera, and $\Delta \Omega$ twice the pixel scale (i.e. the effective spatial resolution).

\subsection{Strawman Instrument Suite}

Based on the above resolution and sensitivity calculations, and the detector technologies associated with different wavebands, we suggest six instrument concepts where PILOT provides unique capabilities that could be exploited with simple camera systems.

- High-spatial resolution imaging in the visible (V-, R-, \& I-bands) with a 45-element ground layer adaptive optics system, providing correction over a moderate field, near to the diffraction limit.

- Wide-field optical imaging (V-, R-, \& I-bands), fully sampled for the natural seeing conditions, so also providing good spatial resolution $\left(\sim 0.25^{\prime \prime}\right)$.

- Near-diffraction-limited imaging in the near-infrared (J-, H-, \& K-bands) over moderately wide-fields, using a tip-tilt system.

- Wide-field, near-infrared (J-, H-, \& K-bands) imaging over the whole sky, using a tip-tilt system, but undersampled with $0.3^{\prime \prime}$ pixel scale.

- High sensitivity, wide-field, diffraction-limited imaging in the thermal part of the near-infrared (K-, L-, \& M-bands), without the need for wavefront correction in L- and M-bands.

- Wide-field, high-sensitivity, diffraction-limited imaging in the mid-infrared (N- and Q-bands), without the need for any wavefront correction.

In addition, PILOT could also operate in the terahertz wavebands, and would be particularly effective if equipped with a (multi-beam) spectrometer or bolometer operating in the 200,350 and $450 \mu \mathrm{m}$ windows.

GLAO system is ideally suited for removal of any ground layer turbulence, and would make it possible to achieve near-diffraction limited images in the optical over a field of view of a degree or more. This would open further science opportunities beyond those implied in this paper using the strawman instrument suite in Table 3.
Table 3 gives strawman instrument specifications for imaging cameras to take advantage of these capabilities, as well as for a single-element terahertz-frequency spectrometer. We assume arrays of the largest sizes typically available commercially in their respective wavebands, in order to provide simple camera systems. However, one can readily envisage butting several arrays together (or using a multi-beam sub-millimetre instrument) if the scientific motivation for covering wider fields is sufficiently high. The wide-field near-IR JHK camera is under-sampled ${ }^{4}$, to give a wide field of view across these wavebands, whereas all the other cameras are Nyquist-sampled at the diffraction limit (or the seeing limit for the wide-field optical imager) for the relevant wavelength $(0.5,1.25,3.8$, and $11 \mu \mathrm{m}$, respectively). Each instrument is also listed with a figure of merit.

\subsection{Wide Field Surveys}

As a relatively small telescope, wide-field instruments can be built for PILOT cheaply in comparison to instruments with the same field of view on 8-m class telescopes. Combined with the sensitivity in the infrared due to the low background and the high-spatial resolution due to the good seeing, this makes PILOT a particularly powerful facility for undertaking wide-field infrared surveys. The field sizes that can be achieved easily surpass those obtainable on larger telescopes, at least without large financial investments. In thermal infrared, while the sensitivity cannot compare with surveys conducted from space, the spatial resolution is several times higher than that of, for example, the space infrared telescope Spitzer. Near-IR surveys

\footnotetext{
${ }^{4}$ It is also possible to recover the full resolution through the technique of 'drizzling' — taking multiple exposures with half pixel offsets between them. This technique has been demonstrated using the wide-field cameras of the HST, which have $0.1^{\prime \prime}$ pixel scale for a $0.1^{\prime \prime}$ telescope point spread function.
} 
Table 4. Prospective Wide Field Infrared Surveys with PILOT

\begin{tabular}{|c|c|c|c|c|c|c|c|c|}
\hline \multirow[t]{2}{*}{ Band } & \multirow{2}{*}{$\begin{array}{c}\lambda \\
(\mu \mathrm{m})\end{array}$} & \multirow{2}{*}{$\begin{array}{l}\text { Resolution } \\
\quad(\operatorname{arcsec})\end{array}$} & \multicolumn{2}{|c|}{ Point source } & \multicolumn{2}{|c|}{ Extended source } & \multirow{2}{*}{$\begin{array}{l}\text { Frame time } \\
\qquad(\min )\end{array}$} & \multirow{2}{*}{$\begin{array}{l}\text { Survey area } \\
\qquad\left(\operatorname{deg}^{2}\right)\end{array}$} \\
\hline & & & (mag) & (Jy) & $\left(\mathrm{mag} \operatorname{arc}^{-2}\right)$ & $\left(\mathrm{Jy} \mathrm{arc}^{-2}\right)$ & & \\
\hline \multicolumn{9}{|c|}{ JHK High Resolution Camera, $0.08^{\prime \prime}$ pixel scale, 5.3' FOV } \\
\hline \multirow[t]{2}{*}{$\mathrm{J}$} & 1.25 & 0.24 & 21.0 & $8(-6)$ & 20.4 & $1(-5)$ & 1 & 90 \\
\hline & & & 23.3 & $1(-6)$ & 22.7 & $2(-6)$ & 60 & 1 \\
\hline \multirow[t]{2}{*}{$\mathrm{H}$} & 1.65 & 0.26 & 19.9 & $1(-5)$ & 19.4 & $2(-5)$ & 1 & 90 \\
\hline & & & 22.2 & $2(-6)$ & 21.7 & $3(-6)$ & 60 & 1 \\
\hline \multirow[t]{2}{*}{$\mathrm{K}$} & 2.3 & 0.3 & 19.8 & $8(-6)$ & 19.5 & $1(-5)$ & 1 & 90 \\
\hline & & & 22.1 & $1(-6)$ & 21.8 & $2(-6)$ & 60 & 1 \\
\hline \multicolumn{9}{|c|}{ JHK Wide Field Camera, $0.3^{\prime \prime}$ pixel scale, $20^{\prime}$ FOV } \\
\hline \multirow[t]{2}{*}{$\mathrm{J}$} & 1.25 & 0.3 & 21.0 & $8(-6)$ & 20.4 & $1(-5)$ & 1 & 1200 \\
\hline & & & 23.3 & $1(-6)$ & 22.7 & $2(-6)$ & 60 & 20 \\
\hline \multirow[t]{2}{*}{$\mathrm{H}$} & 1.65 & 0.3 & 19.9 & $1(-5)$ & 19.4 & $2(-5)$ & 1 & 1200 \\
\hline & & & 22.2 & $2(-6)$ & 21.7 & $3(-6)$ & 60 & 20 \\
\hline \multirow[t]{2}{*}{$\mathrm{K}$} & 2.3 & 0.3 & 19.8 & $8(-6)$ & 19.5 & $1(-5)$ & 1 & 1200 \\
\hline & & & 22.1 & $1(-6)$ & 21.8 & $2(-6)$ & 60 & 20 \\
\hline \multicolumn{9}{|c|}{ KLM Camera, $0.23^{\prime \prime}$ pixel scale, $4^{\prime}$ FOV } \\
\hline \multirow[t]{2}{*}{$\mathrm{K}$} & 2.3 & 0.3 & 19.8 & $8(-6)$ & 19.5 & $1(-5)$ & 1 & 50 \\
\hline & & & 22.1 & $1(-6)$ & 21.8 & $2(-6)$ & 60 & 1 \\
\hline \multirow[t]{2}{*}{$\mathrm{L}$} & 3.8 & 0.42 & 14.8 & $3(-4)$ & 14.8 & $3(-4)$ & 1 & 50 \\
\hline & & & 17.1 & $4(-5)$ & 17.1 & $4(-5)$ & 60 & 1 \\
\hline \multirow[t]{2}{*}{ M } & 4.8 & 0.52 & 13.4 & $8(-4)$ & 13.6 & $4(-4)$ & 1 & 50 \\
\hline & & & 15.7 & $1(-4)$ & 15.9 & $5(-5)$ & 60 & 1 \\
\hline \multicolumn{9}{|c|}{ NQ Camera, $0.7^{\prime \prime}$ pixel scale, $6^{\prime} F O V$} \\
\hline \multirow[t]{2}{*}{$\mathrm{N}$} & 11.5 & 1.2 & 7.6 & $3(-2)$ & 8.7 & $1(-2)$ & 1 & 100 \\
\hline & & & 9.9 & $4(-3)$ & 11.0 & $2(-3)$ & 60 & 2 \\
\hline \multirow[t]{2}{*}{ Q } & 20 & 2.1 & 4.0 & $8(-1)$ & 5.8 & $2(-1)$ & 1 & 100 \\
\hline & & & 6.3 & $1(-1)$ & 8.1 & $2(-2)$ & 60 & 2 \\
\hline
\end{tabular}

Prospective wide-field surveys that could be undertaken with PILOT using the strawman instruments listed in Table 3, each with one month of telescope time devoted to it. Sensitivities are given for point source and extended sources, for a $5 \sigma$ detection in the time per frame listed. The area surveyed assumes a $25 \%$ efficiency over the observing period. The spatial resolution listed is the sum in quadrature of the seeing and diffraction limit, as in Table 1.

conducted from space, for instance with NICMOS on the Hubble Space Telescope (HST), suffer from small fields of view. Hence PILOT can readily probe large parts of parameter space not being tackled with other facilities, lying between the deep, pencil-beam surveys being conducted with some 8-m telescopes, and the shallow, low-resolution all-sky surveys conducted using facilities such as 2MASS. Table 4 lists some prospective surveys that could be undertaken using PILOT with the strawman instrument suite listed in Table 3, and sensitivities adopted from Tables 1 and 2 . These surveys are still relatively modest in observing time, requiring one month of data (assuming a 25\% data gathering efficiency over the period). Depending on the importance assessed to a particular science objective, it would be relatively easy to extend them for additional instrumentation costs, for instance by providing dichroic beamsplitters and additional arrays to survey at two wavelengths simultaneously, or by butting several arrays together to increase the field of view, or simply by conducting the survey over an entire observing season, rather than just one month, to increase the survey area.

We now compare these prospective surveys using PILOT to some other planned wide-field surveys. The
UKIDSS survey on the $4 \mathrm{~m}$ UKIRT on Mauna $\mathrm{Kea}^{5}$ will cover somewhat larger areas in the northern sky in $\mathrm{K}$ band as listed for the wide-field camera in Table 4, but to one magnitude shallower in depth (18.4 mag for $4000 \mathrm{deg}^{2}$ and $21 \mathrm{mag}$ for $35 \mathrm{deg}^{2}$ for SNR = 5). The planned survey on the dedicated 4-m VISTA telescope on Cerro Paranal in Chile ${ }^{6}$ will reach a magnitude deeper in K-band for its widest field component $\left(20.5 \mathrm{mag}\right.$ for $5000 \mathrm{deg}^{2}$, $\mathrm{SNR}=5$ ), but will reach a similar limit for its intermediate field (21.5 mag for $100 \mathrm{deg}^{2}$ ). Both these planned survey facilities, however, will dedicate several years of telescope time to these programs, compared to a few months for a similar survey with PILOT. In the case of the VISTA telescope it requires constructing a $1 \mathrm{deg}^{2}$ field of view camera to perform the task, and the survey will take over a decade to complete. The spatial resolution will also be more than a factor of two lower than could be achieved with PILOT.

The 2MASS near-IR sky survey ${ }^{7}$ reached magnitude limits of $\mathrm{J}=16.7, \mathrm{H}=15.9$, and $\mathrm{K}=15.1(\mathrm{SNR}=5)$, but with $2^{\prime \prime}$ spatial resolution, about 4 mag worse than would

\footnotetext{
${ }^{5}$ wWw.ukidss.org

${ }^{6}$ www.vista.ac.uk

${ }^{7}$ www. ipac.caltech. edu/2mass
} 
be achieved with PILOT, but covering virtually ( $~ 95 \%)$ of the sky. The GLIMPSE survey being conducted with the cryogenically cooled Spitzer space infrared telescope (Benjamin et al. 2003), also with $\sim 2^{\prime \prime}$ resolution, will survey $2^{\circ} \times 120^{\circ}$ of the Galactic plane in the thermal-IR, achieving $\mathrm{SNR}=5$ for magnitudes of $15.5,14.6,12.7$, and 11.4 at $\lambda=3.6,4.5,5.8$, and $8.0 \mu \mathrm{m}$, respectively. The shortest two of these wavelengths are similar to those of the L- and M-bands, for which PILOT's equivalent limits for a wide-field survey are $\sim 1$ mag worse, although with four times higher spatial resolution.

No wide-field, high-spatial resolution surveys are planned at all for the thermal-IR L- and M-bands. While the depths that could be reached with PILOT would be shallower than at K-band, this area of parameter space has so far barely been explored because of the poor sensitivity obtainable from temperate sites. Spitzer/GLIMPSE will provide deeper surveys over selected areas of the sky, albeit at significantly worse spatial resolution, but there is a clear role here for the kind of survey that PILOT could undertake at L- and M-bands.

\subsection{Sub-Millimetre Science with PILOT}

The principal areas of sub-millimetre research are the study of broadband thermal radiation, from either the cosmic microwave background or cool $(5-50 \mathrm{~K})$ dust, and atomic and molecular spectral line emission from gas at temperatures from about ten to several hundred degrees $\mathrm{K}$. While such sub-millimetre wavelength science is not the prime focus for PILOT, the telescope will work perfectly well in these wavebands. That a $2 \mathrm{~m}$ diameter primary mirror is sufficient for state-of-the-art sub-millimetre research is evidenced by many publications arising from the AST/RO, VIPER, and DASI telescopes (e.g. see Stark 2002). PILOT's optics and design could be arranged so as to accommodate one of the multi-pixel heterodyne or bolometer arrays currently being developed by numerous groups (e.g. Payne 2002).

At the South Pole it has been shown that terahertz windows open for some 50 days per year (Chamberlin et al. 2003), a figure likely to be significantly bettered at Dome $\mathrm{C}$, where the water vapour content is lower still. Considering the huge reductions in cost, there remain many sub-millimetre projects best engaged with ground-based telescopes rather than in space. For example, the USA is developing a 10-m telescope (the South Pole Telescope, Ruhl et al. 2004) for the sole purpose of exploiting the South Pole's sub-millimetre sky to survey for the Sunyaev-Zel'dovich effect in galaxy clusters.

\section{Operating Regimes for Antarctic Telescopes}

In this section we consider how the conditions in Antarctica translate best into performance gains for telescopes sited there, in comparison with temperatelatitude facilities.
Assuming equal telescope performance (and ignoring atmospheric transparency differences), the integration time, $T$, needed to reach a given sensitivity limit scales as $B(\theta / D)^{2}$ between two telescopes placed at different sites, where $B$ is the background emission, $\theta$ is the spatial resolution, and $D$ the telescope diameter. This then provides for three different operating regimes that should be considered when comparing the performance of an Antarctic telescope to that of a temperate-latitude facility:

- When the diffraction limit is obtained, $\theta \propto 1 / D$, so that $T$ is proportional to $B / D^{4}$. In Antarctica, this regime applies in the thermal-IR, at $3 \mu \mathrm{m}$ and longer wavelengths, when the diffraction limit for a 2-m telescope exceeds the median seeing.

- For observing extended-sources, when the source size $\theta$ is the same between sites. Then $T$ is proportional to $B / D^{2}$. This applies in any regime where $\theta$ is greater than the seeing or diffraction limit. Since the background drop is typically $\sim 20$ times in the $3-5$ and $8-13 \mu \mathrm{m}$ windows, an Antarctic 2-m is more sensitive than a temperate 8-m telescope for imaging extended sources.

- For the seeing-limited regime, performance comparison depends on the achievable spatial resolution, after wavefront correction. This varies significantly with both wavelength and site, as well as on the performance of the AO system being used. It also depends critically on the isoplanatic angle within which any wavefront correction may be made. For instance, in the near-IR, diffraction limited operation can be obtained from both Mauna Kea and Dome C, but the isoplanatic angle is significantly larger at Dome C. In the optical regime, performance close to the diffraction limit can only be obtained using an AO system on an Antarctic telescope; for the temperate latitude telescope only the seeing limit will be achieved, since the site conditions will not allow an $\mathrm{AO}$ system to operate. In this case, a 2-m telescope at Dome $\mathrm{C}$ can be more sensitive for point source imaging in the optical than an $8-\mathrm{m}$ at a temperate site, within the isoplanatic angle. The science focus in this regime will be on projects where the angular resolution is critical to achieve a new result.

There is also a fourth regime which applies:

- In wavebands where the atmospheric transmission makes observations virtually impossible from the temperate site, but feasible from Antarctica, then completely new kinds of investigation can be considered. This applies in the mid-infrared regimes, from 17 to $40 \mu \mathrm{m}$ and in the far-infrared terahertz windows, especially near $200 \mu \mathrm{m}$. From the very highest site on the Antarctic plateau, Dome A, further windows open up, for instance, near 60 and $150 \mu \mathrm{m}$.

The power of an Antarctic 2-m for science, then, is not predicated on its ability to perform just a single experiment well. An Antarctic 2-m telescope provides a versatile tool, able to tackle science programs in a variety of regimes, each using relatively simple (and inexpensive) 
instrumentation. The science program for PILOT thus revolves around its operation as a community facility, and not just that of a dedicated survey facility. In the sections ahead we outline some of the programs that could be undertaken with such a telescope, taking advantage of the range of performance gains that we have quantified.

\section{Science Programs for PILOT}

In this section we discuss science programs that could be carried out using the PILOT telescope, operating under the conditions prevalent at Dome C (discussed in Section 2), with the performance specifications described in Section 4.1 and the instrument suite described in Section 4.2. These fall into three principal categories:

- our solar system and others,

- our galaxy and its environment,

- our universe and its evolution.

\subsection{Our Solar System and Others}

\subsubsection{The Characterization of Orbital Debris Around the Earth}

Since the launch of the first artificial satellite into orbit in 1957, vast numbers of objects have been placed in earth orbit. Over time there have been collisions and explosions of various rocket bodies and casings, ejected hatches, bolts, fuel, and coolants, virtually filling the orbital sphere with debris, ranging in sizes from metres to just a few microns. These objects now present a significant hazard to manned and unmanned space flights, yet the nature and distribution of the global debris population remains poorly quantified.

It is therefore of great interest to characterize debris objects, their sizes, heights, and altitudes. By observing these objects at various sun-illumination phase angles their albedo can be calculated, and when combined with their brightness this yields their size. Variability in brightness also provides information about the object spin rate and stability, and thereby drag and moment of inertia.

Observing satellite and debris from the Antarctic plateau provides a number of potential advantages to temperate locations. As the majority of satellites and debris of interest lie in polar orbits, there is a natural concentration of objects at the highest latitudes. This provides higher observing efficiency for may programs and allows objects of high interest to be observed on every orbit. Since the orbits precess in longitude, at mid-latitude sites some targets are only sporadically available. Furthermore, the extended terminator period at Antarctic latitudes means that satellites can be tracked in every orbit for long periods of time. This capability opens new avenues for detecting, tracking, and characterizing orbital debris. The excellent seeing and infrared performance of an Antarctic-based telescope also allows smaller and fainter targets to be tracked with multi-spectral capability, further enhancing the ability to determine the composition of the debris. Knowing the material type (from spectral information) makes it possible to more accurately assess the crosssectional area of the object, providing for accurate orbit predictions and longevity of predictions.

\subsubsection{High Resolution Planetary Imaging}

By using the selective imaging (or 'lucky imaging') technique (Baldwin et al. 2001; Dantowitz, Teare, \& Kozubal 2000), it should be possible to obtain diffraction-limited planetary images at any wavelength using a 2-m telescope, giving results comparable with the $H S T$. The images can be used for general monitoring of changes to planetary atmospheres and surfaces. Some specific projects that could be carried out include:

- Studies of the atmospheric circulation of Venus in the lower cloud layer. By observing Venus at 1.7 or $2.3 \mu \mathrm{m}$ the cloud structure can be seen, silhouetted against the thermal radiation from the lower atmosphere. Continuous monitoring of cloud motions at high spatial resolution would provide valuable data that could be compared with the predictions of atmospheric general circulation models (GCMs) for Venus. Currently such observations (e.g. Chanover et al. 1998) are limited by the short duration that Venus can be observed from any one site, and by the limited spatial resolution of the images.

- Studies of the Venus oxygen airglow. Imaging of Venus at $1.27 \mu \mathrm{m}$ will enable the strong (mega-Rayleigh), and highly variable, molecular oxygen airglow emission to be observed from the Venusian upper atmosphere. Continuous monitoring of variations in its intensity and spatial structure would place constraints on the dynamics and chemistry of the upper atmosphere.

- Mars surface pressure imaging. By observing Mars in narrow-band filters that isolate the $2 \mu \mathrm{m} \mathrm{CO}_{2}$ bands it should be possible to image the surface pressure distribution (Bailey et al. 2004). This would allow monitoring of weather systems on Mars and provide data that can then be used to test Martian atmospheric GCMs (Forget et al. 1999).

This program of planetary imaging takes advantages of a number of facets of the Antarctic environment: good seeing, the slow seeing variation timescale, 24 hour coverage, low daylight sky brightness, especially near the Sun, combined with good daytime seeing (as Venus must be observed in daylight), and low water vapour.

It requires imaging in broadband filters at any wavelength from 0.3 to $2.5 \mu \mathrm{m}$, and through selected narrowband IR filters. Diffraction-limited resolution is essential for its success (i.e. $0.06^{\prime \prime}$ at $0.5 \mu \mathrm{m}$ and $0.25^{\prime \prime}$ at $2 \mu \mathrm{m}$ ), but is obtained automatically by the selective imaging technique, without even the need for wavefront correction. Continuous short (10-50 ms) exposures are needed, with real-time processing to select and stack frames. Continuous monitoring is needed for several days, at time resolutions of around a minute. For Venus and Mars, in particular, observations are restricted to favourable opportunities when the planets are in the south and close to 
conjunction and opposition, respectively. Venus has to be observed in daylight, which in Antarctica means the summer time. The project is likely to require a specially designed instrument that can provide short exposure times and fast frame rates.

\subsubsection{Imaging and Photometric Follow-Up of Transiting Planet Candidates}

Transit searches attempt to detect close-orbiting extrasolar planets by detecting the periodic dip in the host star's lightcurve as the planet transits it. They are generally performed with wide-field cameras looking at crowded fields. However, $\sim 90 \%$ of candidates detected by such searches will actually be eclipsing binary stars, either undergoing grazing eclipses, or with the depth of the eclipse diluted by a third, blended star (Brown 2003).

High-spatial resolution imaging and multi-colour photometry provide efficient ways to determine the nature of the transiting system for two reasons. Firstly, stars blended in the wide-field search images can be resolved, allowing the star hosting the transiting object to be identified and so the true (i.e. undiluted) transit depth measured. Secondly, the host star's colours yield estimates of its size and mass, and thus constrain models of the transiting system (Drake \& Cook 2004). Many binary systems can be identified by the colour dependence of the eclipse shape, even when diluted (Tingley 2004).

PILOT provides a suitable facility for such observations because of the high spatial resolution obtainable at Dome $\mathrm{C}$ and the ability to provide guaranteed follow-up of candidates with periods of up to a few weeks. The low scintillation and small variation in airmass also improves the photometric precision. It should be possible to improve from the current 2-5 milli-magnitude rms errors of widefield transit searches to significantly better than 1 millimagnitude precision. The scintillation and Poisson-noise limits for a 1-minute exposure on a V $=13$ star with PILOT are each $\sim 0.3$ mmag (based on equations in Kjeldsen \& Frandsen 1992). The higher signal-to-noise ratio of light curves thus obtained would provide tighter constraints on the physical properties of the transiting system. The good access to the Galactic plane is also a benefit as that is where most transits searches are looking. Candidate sources will also generally be bright enough to be used as their own reference stars for $\mathrm{AO}$ correction.

Imaging would be primarily in the V- and I-bands, followed later possibly by imaging in the near-IR J-, H-, and $\mathrm{K}$-bands to constrain stellar types. The science requires the highest possible spatial resolution that can be achieved in the visible. To be truly advantageous over other telescopes, this requires achieving better than $0.5^{\prime \prime}$ resolution. A field of view of at least $1-2^{\prime}$ across around each candidate star is needed. Candidates will generally be near the Galactic plane or in other crowded fields, such as clusters.

For each candidate, a minimum of two complete transit events would need to be observed. Observation at half-period intervals can also be used to record shallow secondary eclipses. A transit event typically lasts $2-5 \mathrm{~h}$, and typical periods will be for 1-7 days. Thus, a total of $\sim 10-20 \mathrm{~h}$ of observing per candidate will be needed, spread over a period of a few days to weeks. The target stars will be relatively bright ( $\mathrm{V} \leq 14 \mathrm{mag})$, so individual exposures will be less than a few minutes.

There are several transit search programs currently underway which are also well located to provide candidates for follow-up by PILOT. These are Vulcan-South (being conducted at the South Pole; Caldwell et al. 2004), the UNSW/APT Planet Search (at Siding Spring Observatory; Hidas et al. 2004), and the All-Sky Automated Survey (at Las Campanas, Chile; Pojmanski 2002).

\subsubsection{Disks and the Earliest Stages of Planet Formation}

As low mass stars form, they pass through a sequence of stages as an accreting disk first forms around a protostar in a molecular cloud, and then is dissipated as the star emerges onto the main sequence (i.e. the familiar Class 0, I, II, and III stages of star formation; see Figure 7). The disks not only appear to play an essential role in the star formation process, funnelling accreting material and retaining angular momentum, they also provide the raw material for planetary formation. If the dust particles which came together to constitute the Earth were spread throughout the proto-solar system as $1 \mu \mathrm{m}$ sized particles in a disk, their emitting area would be $\sim 10^{13}$ times larger! Disks are thus far more readily observed than the planetary systems they evolve into. Identifying disks, and studying their evolution, therefore provides both a probe of the star formation process, and insights into the production of planetary systems.

Disks are most readily studied in the thermal infrared $(\lambda>3 \mu \mathrm{m}$; see Figure 8$)$. These wavelengths not only penetrate to the depths of cloud cores, but also enable the embedded population to be distinguished from background stars. The warm (few hundred Kelvin) disks emit strongly at $\lambda>3 \mu \mathrm{m}$, and thus are readily distinguished from reddened stars in colour-colour diagrams that extend into the thermal infrared (e.g. $[\mathrm{H}-\mathrm{K}] /[\mathrm{K}-\mathrm{L}]$ ). NearIR colour-colour diagrams (e.g. $[\mathrm{J}-\mathrm{H}] /[\mathrm{H}-\mathrm{K}]$ ), while relatively easy to construct because of the better sensitivities available, show only small IR excesses from these disks, which are readily confused with reddening. NearIR surveys also fail to identify the most deeply embedded sources.

Limited work has so far been undertaken at L-band $(3.8 \mu \mathrm{m})$ as achievable sensitivities are typically $4-5 \mathrm{mag}$ worse than at K-band $(2.2 \mu \mathrm{m})$ from temperate observing sites. However, this defect can be remedied using an Antarctic telescope because of the reduced thermal background. As an illustration of this, Figure 9 shows how the two colour-colour diagrams differ for the galactic star forming region RCW 57 in the (from Maercker, Burton, \& Wright 2005). These data were obtained using the $60 \mathrm{~cm}$ SPIREX telescope at the South Pole. There is a clear improvement between using just near-IR $(1-2.2 \mu \mathrm{m})$ data 
and also using thermal-IR data $(3.8 \mu \mathrm{m})$ in the ability to discern the presence of disks. Only a few of the stars identified as having an IR excesses through use of the L-band data would be so identified if just the near-IR data were available for this task. PILOT could be used to survey regions of star formation to search for IR-excesses in this manner, using the fluxes measured in the J-, H-, K-, and L-bands to determine which sources likely had disks around them.

\subsubsection{Planetary Microlensing in the Inner Galaxy}

Gravitational microlensing occurs if the light ray from a 'source' star passes sufficiently close to a massive foreground 'lens' object so that its path is bent, or lensed, into multiple images (see Figure 10). These images cannot be directly resolved but, as the lensing star moves across the line of sight, the total amplified light from the source star can be measured and this generates a symmetrical light curve profile that is now well recognized (Paczynski 1986). If the lens star has a planetary companion, additional lensing may occur, producing a perturbation in the light curve (Mao \& Paczynski 1991; Gould \& Loeb 1992). A positive demonstration of this effect was recently reported by the MOA and OGLE groups, where an observed 7 day perturbation in the light curve of a microlensing event was attributed to a 1.5 Jupiter mass planet in an orbit around a 0.3 solar mass main sequence star with an orbital radius of $\sim 3 \mathrm{AU}$ (Bond et al. 2004).

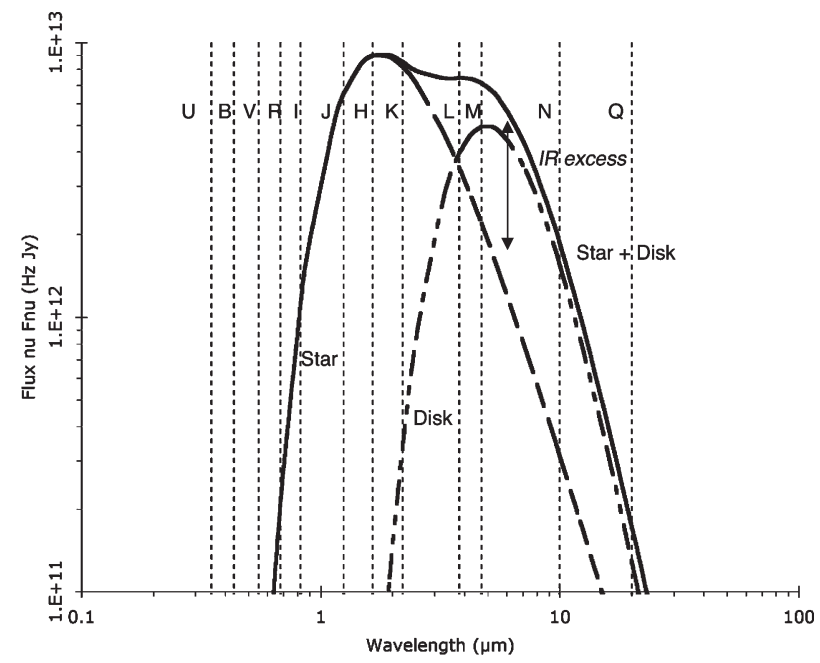

Figure 8 An illustrative example of IR-excess. The Figure shows the spectral energy distribution from a $6000 \mathrm{~K}$ star the same size as the Sun and $150 \mathrm{pc}$ away, surrounded by an edge-on $500 \mathrm{~K}$ disk, of radius $5 \mathrm{AU}$ and height $0.1 \mathrm{AU}$. There is $10 \mathrm{mag}$ of visual extinction to the star, and the thermal emission from the disk is presumed to become optically thin at $\lambda=2 \mu \mathrm{m}$ (with a $\lambda^{-1.7}$ extinction law and emissivity index $\beta=2$ for the grains). The curves show the emission from the star, the disk and their sum. The vertical dashed lines indicate the centres of the photometric passbands from the UV to the mid-IR (i.e. U- to Q-bands). The excess emission from the disk is only weakly discernible at $\mathrm{K}$-band $(2.2 \mu \mathrm{m})$, but is clearly distinguished for observations in L- and M-bands (3.8 and $4.7 \mu \mathrm{m})$. Adapted from an illustration in Lada (1999a).
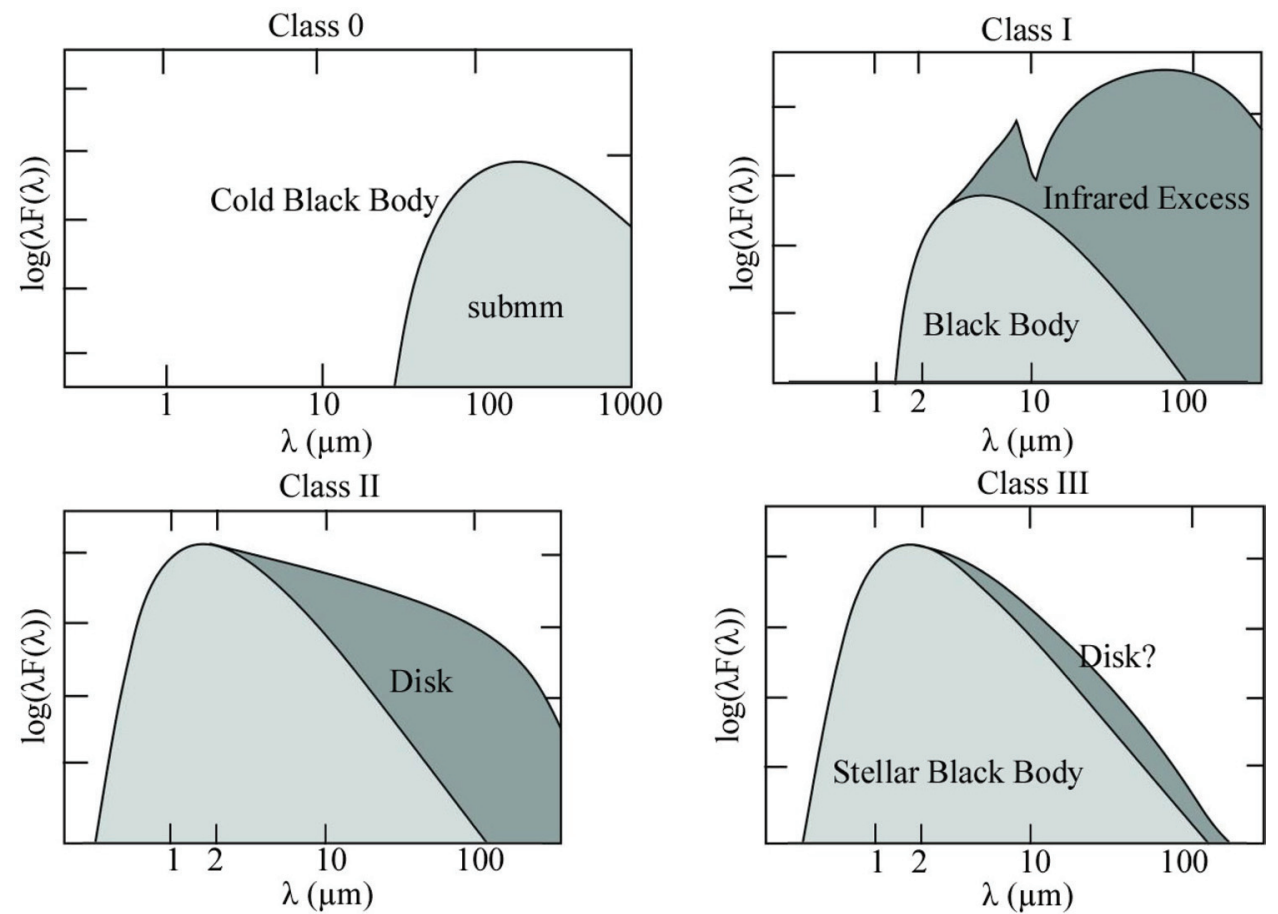

Figure 7 Illustrative sketches show the evolution of the spectral energy distribution during low mass star formation. Initially the core is cold, $T \sim 20-30 \mathrm{~K}$, peaking in the sub-millimetre (Class 0 ). An infrared excess appears and peaks in the far-IR, the emission from a warm envelope heated by the accretion luminosity (Class I). The peak shifts to the mid- and near-IR as a disk forms (Class II). Its spectral shape depends on whether the disk is passive (merely re-processing the radiation from the central star) or active (also kept hot by ongoing accretion). Finally, the disk dissipates (Class III). As is apparent, all stages of disk evolution are best studied at wavelengths longer than $3 \mu \mathrm{m}$; i.e. in the thermal infrared (adapted from Lada 1999b). 
Planetary microlensing is extremely difficult to detect in practice, even though the detection rate of microlensing events in the galactic bulge is high. In general, the planetary perturbations in the light curve are very short lived (occurring on timescales ranging from a few days to a few hours), and can occur anytime during the typically 40 day timescale of a microlensing event. The chances of catching these perturbations can be improved by focussing on the the special class of events where the lens star moves into near perfect alignment with the background star resulting in very high magnifications, in excess of 50. In these events, the planetary perturbations occur within a $24-48$ hour period centred on the time of peak amplification (Griest \& Safizadeh 1998). Furthermore, the sensitivity to planets is greatly enhanced, as was demonstrated in actual observations of high magnification events. In MACHO 1998-BLG-35, which peaked at an amplification of 80, a small perturbation was observed just on the threshold of detectability, probably due to a planet of between 0.5 and 1.5 earth masses (Rhie et al. 2000; Bond et al. 2002). More recent observations of the event MOA 2003-BLG-32 (see Figure 11), which peaked at an extremely high magnification of more than 500, also demonstrated the high planetary sensitivity in these events (Abe et al. 2004). In this case, no planetary perturbations were seen, but earthmass planets were excluded from about 2.3-3.6 AU, and mars-mass planets close to the Einstein ring.

To fully realise the sensitivity to planets in high magnification events, it is necessary to obtain densely sampled uninterrupted observations during a $24-48 \mathrm{~h}$ period. This is currently attempted using a network of collaborating telescopes around the globe. The microlensing survey groups, MOA and OGLE, find around 6-12 high magnification events each year. However, most of these do not receive the required dense sampling - usually because of a combination of unfavourable weather and the lack of a telescope at a suitable longitude at the critical time. Indeed, the event MOA 2003-BLG-32 is the only event to date for which the peak was reasonably fully monitored. In this event, the main observations were made (see Figure 11) with a 1-m telescope towards the galactic bulge from a site, at latitude $+30^{\circ} \mathrm{N}$, with average seeing, yet good sensitivity to Earth-mass, and some sensitivity to Mars-mass, planets was achieved.

PILOT would be ideally suited as a follow-up telescope for high magnification events. The clear skies would greatly improve the 'catchment' of high magnification events alerted by the survey groups. Also, for each event, 'baseline' measurements could be taken at times when the background star is unamplified. Most high magnification events have very faint source stars and the good seeing at Dome $\mathrm{C}$ would allow exposures to achieve a depth greater than could be obtained at similar sized telescopes elsewhere. The source stars typically have $\mathrm{I} \sim 22$ and $\mathrm{V} \sim 23 \mathrm{mag}$, well within the capability of PILOT. Also, because of the small seeing disk, the baseline flux measurements would be much less affected by blending from contamination of nearby stars.

Over a 3-4 year period, 30-40 high magnification events could be monitored at sensitivities comparable to, or better than, MOA 2003-BLG-32. Approximately 300 hours of telescope time would be required per annum. This would provide good statistics on the abundances of gas giants and ice giants, as well as rough statistics on how common terrestrial planets may be. Such results would not be possible using telescopes elsewhere on the globe.
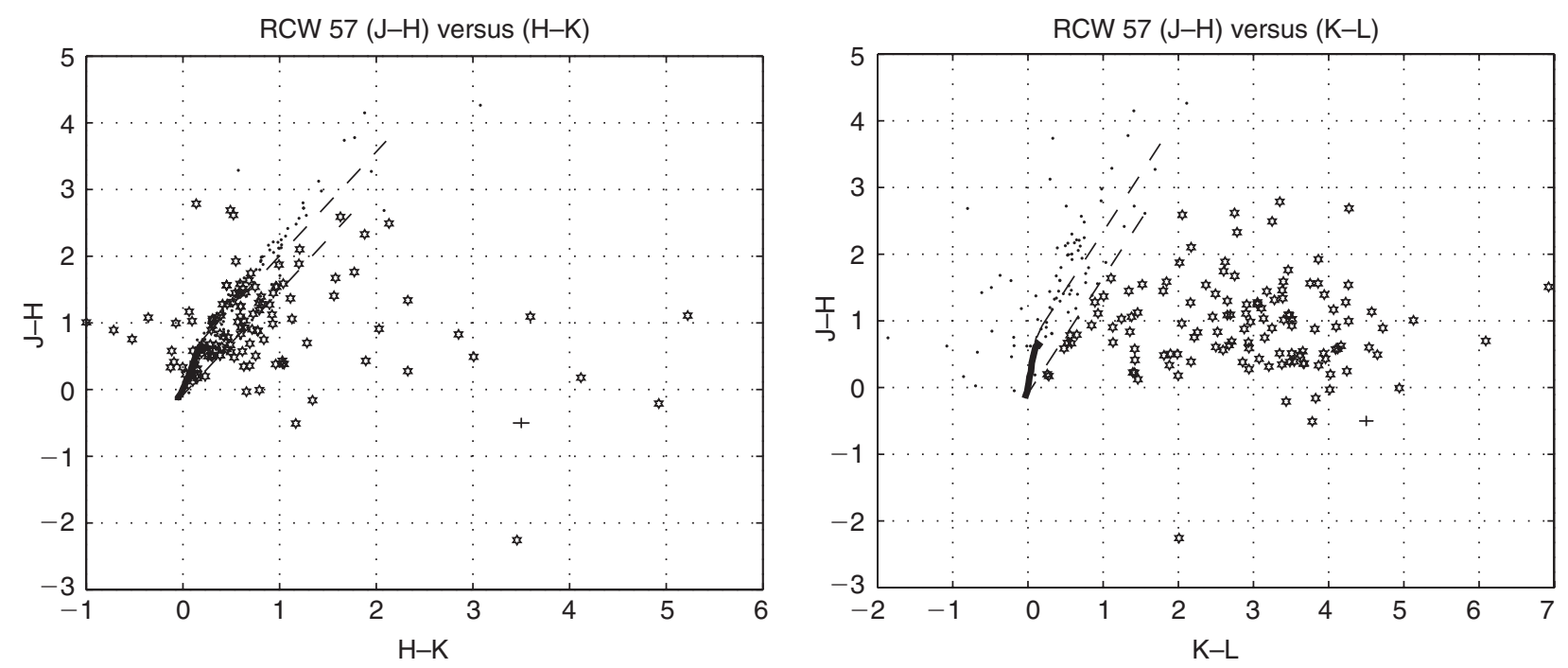

Figure 9 Two infrared colour-colour diagrams for the star forming region RCW 57 constructed from near-IR data obtained with 2MASS (JHK) and SPIREX (L-band) (Maercker, Burton, \& Wright 2005). The near-IR colour-colour plot is shown on the left (J-H versus H-K) and repeated for the same sources using thermal-IR data on the right ( $\mathrm{J}-\mathrm{H}$ versus $\mathrm{K}-\mathrm{L}$ ). The main sequence is shown by the solid line, with reddening vectors up to $A_{V}=30 \mathrm{mag}$ by the dashed lines. Star-shaped symbols show sources identified as having a disk from the L-band data, whereas dots indicate sources which do not show evidence for disks. While a few sources show strong excesses at K-band, it is clear that this comprehensive separation of disks from reddened stars could not be carried out using the JHK data alone. 


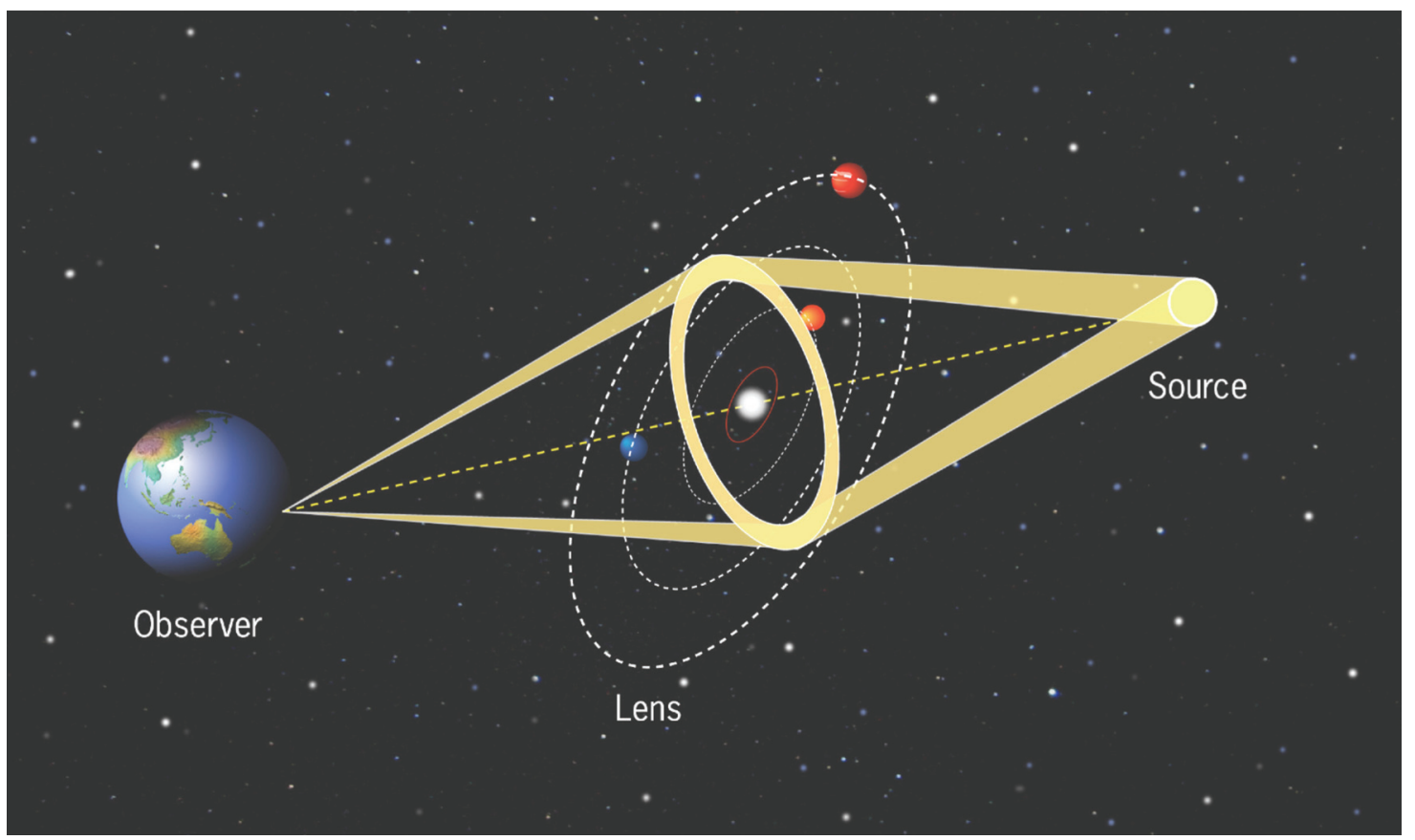

Figure 10 Illustration of gravitational microlensing. In this example, the alignment of the lens and the source is perfect, leading to the formation of a complete Einstein ring. For Galactic microlensing, the radius of the Einstein ring is typically $\sim 2$ AU. Planets orbiting the lens star may therefore perturb the Einstein ring. Several microlensing events are detected annually with alignments of $\sim 0.01 \mathrm{AU}$. These events provide significant sensitivity to the presence of Earth-mass planets.

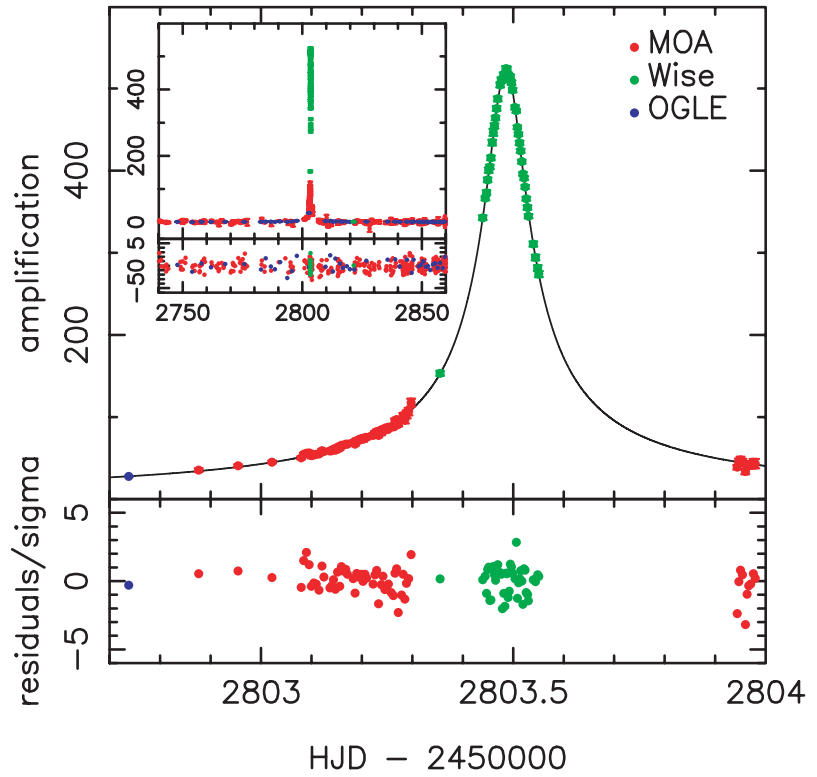

Figure 11 Light curve of the high magnification microlensing event MOA 2003-BLG-32 obtained by MOA and OGLE and the Wise observatory (Abe et al. 2004). Observations of similar events from Dome $\mathrm{C}$ would allow one to obtain a seamless uninterrupted light curve using a single instrument.

If a dedicated wide-field telescope was built for microlensing studies at Dome $\mathrm{C}$, equipped with a large format array camera, this project could be extended to determine how common all these different types of planets are. Such a telescope would ideally provide both optical (V- \& I-bands) and near-IR (J-, H-, \& K-bands) coverage (see Bennett et al. 2004). A field-of-view of $\sim 1.5 \mathrm{deg}^{2}$ would suffice, with two fields of $\sim 0.75 \mathrm{deg}^{2}$, continuously sampled in the two passbands in a chopping mode at intervals of $20 \mathrm{~min}$ or less. This could be achieved with a giga-pixel camera with a pixel size $\sim 0.1^{\prime \prime}$. Several thousand microlensing events could be monitored over a three to four year period - all with high density, uninterrupted sampling measurements. If systems similar to our own solar system are common, such a survey could detect $\sim 50$ terrestrial planets, $\sim 100$ ice giant planets, and $\sim 1000$ gas giant planets.

\subsection{Our Galaxy and its Environment}

\subsubsection{Stellar Oscillations}

A star is a gaseous sphere and will oscillate in many different modes when suitably excited. The frequencies of these oscillations depend on the sound speed inside the star, which in turn depends on properties such as density, temperature and composition. The Sun oscillates in many modes simultaneously. By comparing the mode frequencies to theoretical calculations (helioseismology), significant revisions to solar models have been made (e.g. Christensen-Dalsgaard 2002). For example, the Sun's convection zone has turned out to be $50 \%$ deeper than previously thought; the helium abundance cannot be as low as is required to reproduce the apparent neutrino flux (a result vindicated by recent evidence from the Sudbury Neutrino Observatory for neutrino flavour oscillations; SNO 2002); the angular velocity does not increase rapidly with depth, thereby removing the inconsistency between planetary orbits and general relativity; and the opacities 
had been underestimated immediately beneath the convection zone. Measuring oscillation frequencies in other stars (asteroseismology) will allow us to probe their interiors in exquisite detail and study phenomena that do not occur in the Sun. We expect asteroseismology to produce major advances in our understanding of stellar structure and evolution, and of the underlying physical processes.

Thanks to the steadily improving Doppler precision provided by modern spectrographs, the field of asteroseismology has finally become a reality. For a recent review, see Bedding \& Kjeldsen (2003). However, to make further gains requires that stars be observed as continuously as possible. It would also be valuable to observe clusters of stars photometrically with extremely high precision. Antarctica offers both of these possibilities and is second only to space as the site for an asteroseismology program. The low scintillation at Dome $\mathrm{C}$, combined with the long time period available for observations, presents an opportunity to carry out asteroseismology on solar-like stars and to achieve a breakthrough in our understanding of the role played in stellar evolution by processes such as convection, rotation, and mixing.

\subsubsection{The Earliest Stages of Massive Star Formation}

Massive star formation drives the star-gas cycle of the Galaxy. It is the crucible through which Galactic evolution is played out, creating the most luminous objects in the Galaxy and driving the material cycles of the elements. It is of particular interest to identify the very earliest stages of massive star formation. However, despite being manifest across the Galaxy on account of their luminosity, the process of massive star formation remains something of a mystery. There are no nearby examples to dissect, unlike low mass star formation, and the processes at work take place on short timescales and in obscured environments that have not been readily accessible to observation.

Massive protostars are so cold that they can only be detected at far-IR and sub-millimetre wavelengths. As they warm up, their spectral energy distributions evolve, becoming visible in the mid-IR and then near-IR wavebands. The earliest stages have been nearly inaccessible to study, since the emission from $20-30 \mathrm{~K}$ objects peaks around $100 \mu \mathrm{m}$. From Dome C, however, measurements could be made at $200 \mu \mathrm{m}$, close to this emission maximum. Moreover, they can then be combined with measurements in the sub-millimetre, at 350, 450, and $850 \mu \mathrm{m}$, spectral windows which are open virtually continuously at Dome $\mathrm{C}$, allowing the energy distributions to be determined in the Rayleigh-Jeans part of the spectrum. Such measurements, since they are at optically thin wavelengths, allow the dust mass to be estimated. The slope of the energy distribution then provides a probe of the dust emissivity, which is a function of the nature of the dust grains (e.g. their size, composition, and distribution). Hence a terahertz frequency spectrometer at Dome $\mathrm{C}$ will provide a new tool for investigating the earliest stages of massive star formation across the Galaxy.
As the cores heat up, an embedded object appears at mid-IR wavelengths. It heats the surrounding dust grains, from which molecules are evaporated, driving a rich organic chemistry in the gas phase, all of which is amenable to investigation through sub-millimetre and millimetre-wave spectroscopy. All but the very coldest massive protostars are visible in the mid-IR, and the spectral features (particularly the silicate dust feature) can be used to measure the column density. The colour variations across the mid-IR window, from 8 to $30 \mu \mathrm{m}$, indicate the evolutionary state of the sources (i.e. how deeply embedded they are). Hence multi-wavelength imaging in the mid-IR window, combined with measurements of the spectral energy distribution of the sub-millimetre emitting cores, provides a method of determining the initial embedded population of massive star forming cores, the luminosity and mass of the dominant sources within them, and their differing evolutionary states. There does not yet exist a paradigm for massive star formation equivalent to the Class 0-III states associated with low mass star formation, and one of the goals of such studies is to identify whether there are characteristic observational signatures associated with each stage. For example, Figure 12 shows model plots of the relative energy distributions of embedded massive protostars, as calculated using the DUSTY code (Ivezić \& Elitzur 1997), overlaid with the atmospheric windows available at Dome C. Such objects will become accessible for study with a telescope such as PILOT, equipped with mid-IR and sub-millimetre instrumentation.

\subsubsection{Spectral Features in the Infrared and their Evolution During Star Formation}

The infrared window from 3 to $30 \mu \mathrm{m}$ contains a plethora of spectral features, many of which trace the physical conditions in the circum-protostellar environment. The main modes of excitation seen through these features are: atomic fine structure lines (diagnostics of ionization), gas-phase rotational/vibrational bands, polycyclic aromatic hydrocarbon (PAH) emission, and solid-state vibrational bands (from dust and ice).

Observations from the Infrared Space Observatory (ISO) satellite have shown that there is a wealth of information to be reaped from mid-IR spectroscopic observations (see Figure 13). The sharply rising continuum flux, with absorption along the line of sight by dust and ice mantle components, is characteristic of heavily embedded massive protostars. These ices provide the raw material for the chemical evolution of the dense cores. As a protostar begins to heat up, it evaporates the ices into the gas phase, beginning the hot molecular core phase of massive star formation. Observation of the ice features therefore probes the state of the coldest and densest regions of molecular clouds, prior to the onset of star formation in them.

The depletion of species onto the dust grains is also an important process in the early evolution of the cores. This is partly because the chemistry of star forming regions 


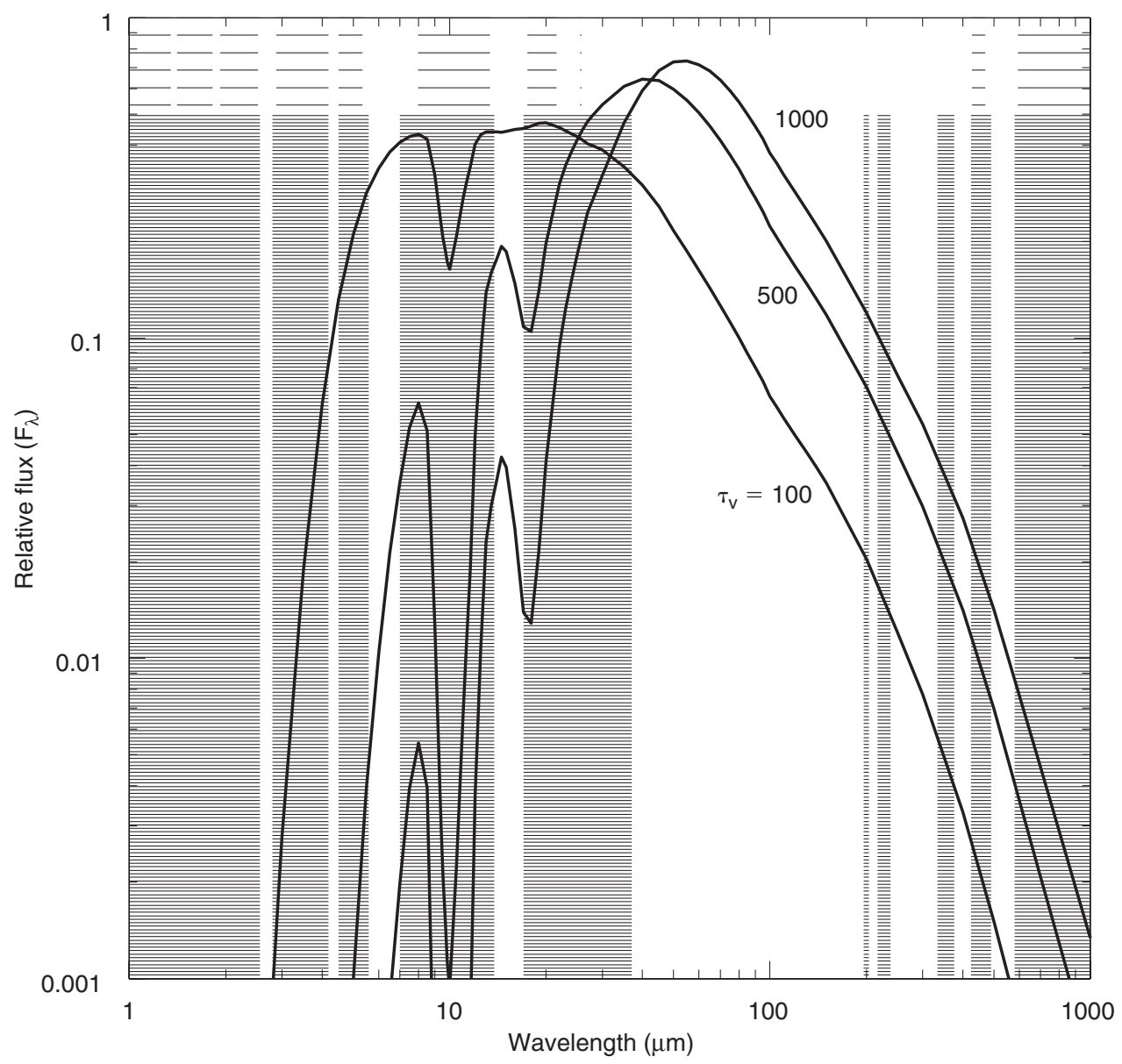

Figure 12 Model spectral energy distributions (SEDs) representative of embedded massive protostars, as a function of the optical depth of the obscuring material to the central star $\left(\tau_{V}=100,500, \& 1000\right)$. It is clear that infrared observations are needed to study such objects. These SEDs have been calculated using the DUSTY code (Ivezić \& Elitzur 1997), for a central source temperature of $30000 \mathrm{~K}$ and an $r^{-1.5}$ density distribution of silicate grains. The features in the spectrum at 10 and $18 \mu \mathrm{m}$ are due to absorption from the silicates. The shaded overlays show the windows accessible from Dome C, and at the top, in lighter shading, from Mauna Kea. They illustrate the new windows that open at Dome C in the mid-IR and sub-millimetre that will facilitate this program.

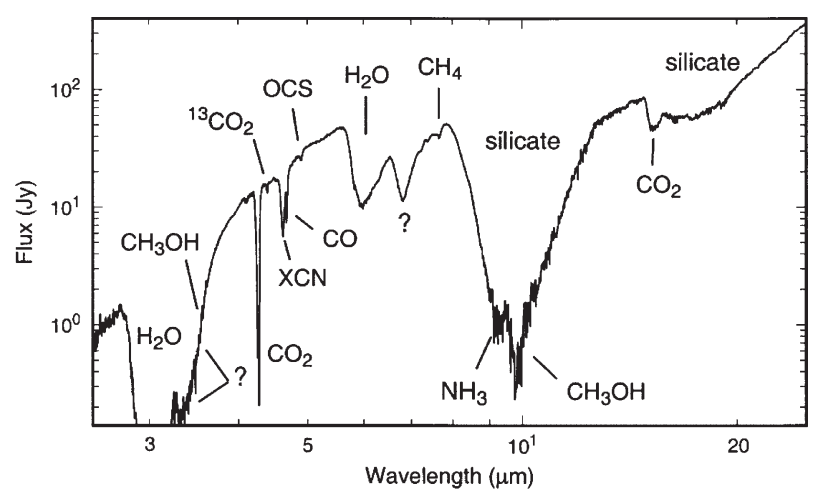

Figure 13 A composite infrared spectrum of W33A, a massive young stellar object, obtained with a resolving power of 500-1000 using several instruments on the $I S O$ satellite (from Gibb et al. 2000). Many of these features are present in the accessible part of the spectrum from Dome C. For instance, ices in the grain mantles produce absorption from $\mathrm{H}_{2} \mathrm{O}$ at 3.1 and $6 \mu \mathrm{m}$, from $\mathrm{CO}$ at $4.7 \mu \mathrm{m}$, from $\mathrm{CH}_{4}$ at $7.7 \mu \mathrm{m}$, from $\mathrm{NH}_{3}$ at $9 \mu \mathrm{m}$, and from $\mathrm{CH}_{3} \mathrm{OH}$ at $10 \mu \mathrm{m}$, as well as silicate absorption at 9.7 and $18 \mu \mathrm{m}$ (see van Dishoeck 2004 for a complete list). There may also be PAH emission features at 3.3 , $6.2,7.7,8.6,11.3$, and $12.7 \mu \mathrm{m}$. is greatly changed as a result, but also because depletion interferes with our ability to study the earliest stages of star formation. If a species depletes onto dust grains it cannot then be used as spectral line probe of the state of the gas where this occurs. Hence, as a result of depletion, the kinematics of the innermost, coldest, densest regions of molecular clouds have not been probed. For instance, even $\mathrm{N}_{2} \mathrm{H}^{+}$, which had been considered as a non-depleter, has recently been found to indeed deplete in B68 (Bergin et al. 2002), so rendering it unsuitable for probing the core.

There appear to be two remaining species which might be used to follow the kinematics of the earliest stages of star formation, the transitions of para $-\mathrm{H}_{2} \mathrm{D}^{+}$at $218 \mu \mathrm{m}$ and ortho- $\mathrm{D}_{2} \mathrm{H}^{+}$at $202 \mu \mathrm{m}$. These ions should be the last to deplete out of the gas in the coldest and densest cores (Bergin, private communication). The two transitions are accessible from Dome C.

The variation in spectral lines features between embedded protostars traces the time-dependent chemistry of the regions, providing an evolutionary clock to classify the earliest stages of star formation. Combined with the derived physical conditions, mid-IR spectral line 
observations provide a powerful and relatively unexplored tool for probing regions of star formation.

The ISO observations shown in Figure 13 are limited by the spatial resolution of the 60 -cm telescope aperture, providing information on only the large scale core properties. Recent high spatial resolution mid-IR observations (e.g. Longmore et al. 2005), show many of the cores that appear as point sources with $M S X$ (a 35 -cm satellite operating in broad bands from 8 to $21 \mu \mathrm{m}$ ) are resolved into multiple sources, altering the interpretation of large scale data. There is a lacuna for larger, ground-based telescopes to extend the spectroscopic observations to higher spatial resolution.

Dome $\mathrm{C}$ is an attractive site for such a telescope, not only because of the significant decrease in the thermal background and the improved transmission and sky stability. The spectrally quiet $30-40 \mu \mathrm{m}$ window that becomes available provides a good continuum baseline, free from the solid state absorption lines that hinder derivation of the optical depth from spectra that only extend to $25 \mu \mathrm{m}$ (i.e. as obtainable from mid-latitude sites).

\subsubsection{A Wide-Field Sub-Millimetre Survey of Star Forming Regions: From Low Mass Protostellar Cores to the Progenitors of OB Stars}

Star formation is a key astrophysical mechanism driving the evolution of the Galaxy. Significant progress has been achieved in our understanding of the formation of low and intermediate mass stars. These stars form from the collapse of dense cloud cores in the molecular interstellar medium of the Galaxy. Two very early evolutionary stages have recently been identified as part of this process: gravitationally bound, starless, pre-stellar cores (e.g. Ward-Thompson, André, \& Kirk 2002), and cold protostars or Class 0 protostars (e.g. André, Motte, \& Bacmann 1999). Yet, several fundamental questions remain open. For instance, how do pre-stellar cores form in the molecular clouds and what governs their collapse and evolution to protostars? What determines the distribution of the initial distribution of stellar mass (i.e. the IMF)?

The formation of high-mass $\left(\mathrm{M}>8 \mathrm{M}_{\odot}\right)$ stars is, in contrast, less well understood. Massive stars are born in heavily obscured regions ( $A_{v}>20 \mathrm{mag}$ ) of cold molecular gas and dust, far away from us (typically a few kpc) and within dense stellar clusters $\left(\sim 10^{4}\right.$ stars $\left.\mathrm{pc}^{-3}\right)$. They also experience short lives $\left(\sim 10^{6}-10^{7}\right.$ years $)$. These observational constraints have limited our knowledge about the processes involved in high-mass star formation (HMSF). An empirical evolutionary sequence has been proposed: massive stars form in hot molecular cores and then evolve to ZAMS stars, that ionise their environment to produce several classes of HiI regions (hyper compact, ultra compact, etc.). Recent (sub-)millimetre observations also claim the discovery of high-mass protostellar objects, which are likely to be protoclusters of embedded young stellar objects (e.g. Minier et al. 2005).

Theoretically, high-mass star formation is problematic. Increasing outward radiative acceleration becomes important for high-mass stars and will ultimately stop accretion. The infall accretion rate must then exceed the outflow rate to produce a high-mass star, i.e. the gravity must overcome the radiative pressure. Two scenarios have been proposed to explain this: HMSF could proceed either through protostellar mergers (Bonnell \& Bate 2002) or through the collapse of a single, supersonically turbulent core with a sufficiently large accretion rate (McKee \& Tan 2003). High-mass, pre-stellar cores are only expected in the second scenario. The main physical processes leading to high-mass stars are then still unclear. We might ask whether massive stars also form through the collapse of a pre-stellar core. Do 'Class 0' high-mass protostars exist?

To address the unanswered questions in these fields of low and high mass star formation, we propose a submillimetre survey to find and classify the cores. Unbiased surveys of Giant Molecular Clouds (GMCs) in the far-IR/sub-millimetre continuum are necessary in order to make a complete census of the pre- and proto-stellar population within star-forming regions. This is because pre-stellar cores, Class 0 protostars, and HMSF protoclusters emit the bulk of their luminosity between 60 and $400 \mu \mathrm{m}$ (André et al. 1999; Ward-Thompson et al. 2002; Minier et al. 2005). The coldest objects have the peak of their spectral energy distribution around $200 \mu \mathrm{m}$.

An unbiased and wide-field survey at 200, 350, and $450 \mu \mathrm{m}$ of nearby regions of star formation will allow us to potentially detect all the pre-stellar cores, protostars and HMSF protoclusters in a given star-forming region. Their mass and luminosity can then be derived via spectral energy distribution modelling, obtaining the early stellar population (i.e. the IMF). To do so, a sample of star forming regions from low mass (e.g. Chamaeleon) to OB star progenitor complexes (e.g. NGC 6334) is needed. A sensitivity to reach a $0.1 \mathrm{M}_{\odot}$ protostar at $0.5 \mathrm{kpc}$ at $200 \mu \mathrm{m}$ is desirable.

Such an investigation benefits considerably from an Antarctic location because observations at 200, 350, and $450 \mu \mathrm{m}$ are possible. The opening of the $200 \mu \mathrm{m}$ window is an exceptional feature as this wavelength is generally unobservable from the ground. The ESA Herschel Space Observatory will observe in the continuum from 60 to $500 \mu \mathrm{m}$ with the PACS and SPIRE instruments. Using a filled bolometer array, as developed for PACS, will greatly facilitate such a survey in Antarctica.

\subsubsection{The Galactic Ecology}

The environment of star forming complexes, which dominate the southern Galactic plane, can be studied in the thermal infrared from 2.4 to $4.1 \mu \mathrm{m}$ through the spectral features emitted from excited gas in these regions. The comparison can be made through studying the emission from three spectral features: $\mathrm{H}_{2}$ emission at $2.4 \mu \mathrm{m}$ in the $v=1-0 \mathrm{Q}$-branch lines, the $3.3 \mu \mathrm{m}$ PAH emission feature, and $\mathrm{Br}-\alpha$ emission at $4.05 \mu \mathrm{m}$. This allows the conditions and extent of the molecular, neutral (i.e. the 
photodissociation or PDR interface) and the ionized gas to be probed. For example, the SPIREX images obtained at the South Pole of the NGC 6334 massive star formation complex (Burton et al. 2000), show shells of fluorescently excited molecular gas surrounding several sites of massive star formation, each $\sim 1 \mathrm{pc}$ apart and spread along a dense molecular ridge. Complex PAH organic molecules pervade the region, and are fluorescently excited by the far-UV radiation that escapes from these stars. These images are displaying the Galactic ecology at work the interaction between young stars and the interstellar medium, in its ionized, neutral, and molecular phases.

The spectral features in the images also provide information on the excitation processes at work in the clouds. Any $\mathrm{H}_{2}$ line emission results from either shocks or UV-fluorescence. The $v=1-0$ Q-branch lines at $2.4 \mu \mathrm{m}$ are both stronger and suffer less extinction than the commonly used 1-0 S(1) line at $2.12 \mu \mathrm{m}$. They emit in the $\mathrm{K}$-dark band, the region of lowest atmospheric thermal emission from Antarctica, and so a particularly sensitive window for observation. Several solid state absorption features are also present, for instance the ice band at $3.1 \mu \mathrm{m}$. Polycyclic aromatic hydrocarbons, organic molecules that are fluoresced by far-UV radiation from the young stars and trace the edge of photodissociation regions, are visible through a spectral feature at $3.3 \mu \mathrm{m}$ (as well as having several features in the mid-IR window). Through these thermal-IR lines, the PDR structure can be imaged at high spatial resolution, unlike other prime PDR tracers, such as the far-IR [CII] $158 \mu \mathrm{m}$ line, which can only provide low resolution maps. Finally, HII and ultra-compact HII regions can be traced in the $\operatorname{Br} \alpha 4.05 \mu \mathrm{m}$ line, even when deeply embedded. These can be compared directly to radio interferometric maps of the same gas to determine the extinction, and to estimate the ionizing luminosity.

\subsubsection{Spectral Line Sub-Millimetre Astronomy}

The formation, structure, dynamics, abundance, composition, and energetics of molecular clouds in the Milky Way and nearby galaxies are all active areas of research. Questions regarding how molecular clouds form, what triggers their collapse, why the process of star formation does not halt as the collapsing gas heats up, which species dominate the cooling, and the role played by magnetic fields all remain significant problems. These issues are best addressed using millimetre and sub-millimetre wavelength observations, since the radiation in these bands contains large numbers of indicative spectral lines which, unlike optical lines, can escape from the dense gas and dust clouds from which they originate.

When molecules in a cloud collide, energy can be transformed from kinetic into rotational, vibrational, and electronic potential energy. It is then released through the emission of photons with wavelengths and intensities characteristic of the species involved, so revealing the abundance, temperature, and density in the gas. The most abundant elements in molecular clouds - hydrogen and helium - are however poor tracers of these conditions as they cannot be collisionally excited to radiate at the low temperatures found in the clouds. On the other hand, carbon, the fourth most common element, can be readily observed in its ionized, neutral, and molecular states, as well as in dust grains, thus sampling the full range of physical conditions found. Nitrogen, the sixth most abundant element, has an important atomic line at $205 \mu \mathrm{m}$, but this has never been observed from the ground.

Neutral carbon has fine structure transitions at $610 \mu \mathrm{m}$ $\left({ }^{3} \mathrm{P}_{1} \rightarrow{ }^{3} \mathrm{P}_{0}\right)$ and $370 \mu \mathrm{m}\left({ }^{3} \mathrm{P}_{2} \rightarrow{ }^{3} \mathrm{P}_{1}\right)$. The $610 \mu \mathrm{m}$ transition is only $23 \mathrm{~K}$ above ground and has a critical density of $1000 \mathrm{~cm}^{-3}$. This implies that it is easily excited and so the [CI] lines will be readily detectable, even when emitted by moderate density interstellar gas exposed only to typical ambient Galactic radiation fields. Excellent atmospheric transmission is required to measure these important lines, however, and so there has been relatively little work done on the neutral lines of carbon compared to the optical (i.e. ionized) lines of the atom. The first detection of [CI $]{ }^{3} \mathrm{P}_{1} \rightarrow{ }^{3} \mathrm{P}_{0}$ in another galaxy (the Large Magellanic Cloud) was made with a $1.7 \mathrm{~m}$ radio-telescope from the South Pole (AST/RO; Stark et al. 1997), and the most extensive mappings of $[\mathrm{CI}]{ }^{3} \mathrm{P}_{2} \rightarrow{ }^{3} \mathrm{P}_{1}$ in any objects have also been made with this instrument (NGC6334 and the Galactic Centre; Martin et al. 2004).

Carbon monoxide (CO), the most abundant molecule after $\mathrm{H}_{2}$, needs to be observed in several rotational $(J)$ levels if the physical conditions are to be inferred from the line strengths. The low- $J$ states are close to local thermodynamic equilibrium (LTE), so their excitation temperatures, $T_{\mathrm{ex}, J}$, are all close to the kinetic temperature, $T_{\text {kin. }}$. Hence the ratio of line brightnesses for transitions between such states is near unity and so independent of $T_{\text {kin. }}$. However, for any temperature and density there will be higher $J$ states that will fail to be in LTE, and so must be sub-thermally excited (i.e., $T_{\mathrm{ex}, J}<<T_{\mathrm{kin}}$, with line ratios $T_{J \rightarrow J-1} / T_{1 \rightarrow 0}$ departing from unity. Thus, measurements of optically thin transitions of $\mathrm{CO}$ from rarer isotopomers (e.g. ${ }^{13} \mathrm{CO}, \mathrm{C}^{18} \mathrm{O}$ ), and from high- $J$ states, are needed to probe the hottest and densest regions in molecular cloud cores. Such measurements require sub-millimetre wavelength observations, and so are wellsuited for an Antarctic telescope.

\subsubsection{Continuum Wavelength Sub-Millimetre Astronomy}

A wide range of astrophysical phenomena can be studied using far-IR and sub-millimetre bolometers, including dust in molecular clouds, hot star-forming cores and stellar remnants, synchrotron emission in our Galaxy, the origin of the far-IR background, and the evolution of IR-luminous galaxies at high redshifts. A wide field instrument on PILOT would complement the new generation of millimetre and sub-millimetre interferometers being built, such as the Atacama Large Millimetre Array (ALMA) in 
northern Chile. Bolometer arrays with thousands of pixels can now be envisaged (e.g. Staguhn et al. 2003), and a strong science case for such arrays on small telescopes has been presented (Stark 2003). By virtue of the extremely dry conditions, a telescope at Dome $\mathrm{C}$ would be well suited for utilizing these bolometer arrays for continuum mapping in the 200, 350, and $450 \mu \mathrm{m}$ atmospheric windows.

\subsubsection{A Survey for Cool Brown Dwarfs and Extrasolar Giant Planets}

Brown dwarfs are sub-stellar mass objects, whose mass is too low for the nuclear fusion of hydrogen to sustain their luminosity over the bulk of their lifetime (though the fusion of lithium, deuterium, and even hydrogen is possible in a brown dwarf, depending on its mass and age). Brown dwarfs are born hot, shining primarily through the release of gravitational potential energy and accretion luminosity, and spend the rest of their lives cooling, emitting in the infrared (e.g. Burrows et al. 2001). As they cool, their spectra change radically, quite unlike stars. Spectroscopically, they can at first look similar to latetype M-dwarfs, they then pass through a L-dwarf stage where warm ( $\sim 1300-2100 \mathrm{~K})$ dust emission dominates, and they finally end in the T-dwarf stage where absorption bands from methane, water, and ammonia dominate (cf. the infrared emission from Jupiter) as they cool further. This means that the spectral type of a brown dwarf depends on both its mass and the age, unlike a main sequence star. Modelling is needed to separate the degeneracy between mass and age for a star of a given surface temperature. Infrared colours (JHKLM-bands from 1 to $5 \mu \mathrm{m})$ can be used to spectrally identify brown dwarfs, though the presence of strong methane absorption bands means that while the $\mathrm{K}-\mathrm{L}$ colour is red (as expected from a cool object), the near-infrared colours (e.g. H-K) of T-dwarfs may be neutral or even blue (as the radiation is forced to shorter wavelengths to escape through spectral windows in the brown dwarf atmospheres). Before such identifications can be made, however, the challenge is to find brown dwarfs - after their first few million years they have cooled so much that they are both faint and emit the bulk of their radiation in thermal infrared wavelengths, and hence are difficult to detect.

Giant extrasolar planets also fall into the same class of objects as brown dwarfs. While their origins are likely quite different (they are formed in the disk surrounding a young stellar object versus a separate gravitational condensation site), physically they are identical objects (although environmental differences, e.g. proximity to a star that provides an external heating source, may result in some different observational characteristics). The study of brown dwarfs therefore overlaps with that of planetary science, and will provide insight into the latter.

The study of brown dwarfs is thus a fundamental part of the study of stars, since they can be formed by the same processes with the only difference being the mass of the resulting object. The future evolution of brown dwarfs is, however, radically different to that of stars, being that of a degenerate body without a central fusion luminosity source. It is less than a decade since the first clear evidence of a brown dwarf was reported (Gliese 229B; Oppenheimer et al. 1995) and there remains much to be learnt about their properties and evolution. While it is now clear that brown dwarfs do not provide for the missing mass needed to account for galactic rotation curves, it is also clear that their number density in the solar neighbourhood is comparable to that of M-dwarfs. The initial mass function is still rising as the mass falls below the fusion-edge defining the main sequence. Isolated brown dwarfs have also been found to be numerous in several galactic clusters. Of particular interest is the occurrence of brown dwarfs in binary systems, for which only limited information is available. Binary frequency decreases with stellar mass, from $\sim 60 \%$ for solar-type stars to $\sim 35 \%$ for M-dwarfs. Furthermore, there are few sub-stellar-mass companions found to solar-type stars in radial velocity surveys targeting planets (the so-called 'brown dwarf desert'; Marcy \& Butler 1998). Understanding why this is the case is important for the theory of star formation, and the cause of the initial mass function, but there are limited statistics so far to provide a clear picture of the degree of binarity in its low mass end. Brown dwarfs also display weather the atmospheric chemistry in their photospheres, which is a strong function of temperature, and changes radically during evolution as the brown dwarf cools. Photometric and spectroscopic variations can be expected due to presence of cloud systems as the sources rotate. Such changes will also be a function of spectral type.

All these facets of brown dwarf behaviour are little understood, as there are still relatively few sources known (a few hundred, dominated by the hotter and younger sources). PILOT, with its sensitivity in the thermal-IR L- and M-bands, is well suited to extend the current surveys, both to greater distances and to the detection of cooler (and thus older, and presumably far more common) brown dwarfs. In particular, a survey at L-band $(3.8 \mu \mathrm{m})$ to a depth of $14.8 \mathrm{mag}$ (as could be reached in $1 \mathrm{~min}$ with PILOT) would provide a similar sensitivity to that reached by the 2MASS survey (Kirkpatrick et al. 1999) in the K-band, but be capable of detecting cooler and redder sources. Table 5 (adapted from Burrows et al. 2001) gives an indication of how far away a $15 \mathrm{M}_{\text {Jupiter }}$ brown dwarf could be found, as a function of its age, in the K-, L-, and M-bands. While such an object can readily be detected when it is young, by the time it has reached $10^{9}$ years in age it can only be found if it is relatively close to the Sun. Note, however, that PILOT would be as sensitive to detecting such objects in the M-band as it is in the $\mathrm{K}$-band, despite the greatly reduced sensitivity, because brown dwarfs this old are so cool. PILOT could therefore be used to conduct the first extensive survey for old, cool brown dwarfs within a few tens of parsecs of the Sun.

The primary advantage of Antarctica for this experiment is the high sensitivity in the L- and M-bands compared to temperate sites. The project described here 
Table 5. Distance to which a $15 \mathrm{M}_{\text {Jupiter }}$ Brown dwarf could be detected

\begin{tabular}{lcccccrrrr}
\hline $\begin{array}{l}\text { Age } \\
(\mathrm{yr})\end{array}$ & $\begin{array}{c}T_{\text {eff }} \\
(\mathrm{K})\end{array}$ & $\begin{array}{c}\text { Spectral } \\
\text { type }\end{array}$ & $\begin{array}{c}\text { K flux } \\
(\mathrm{Jy})\end{array}$ & $\begin{array}{c}\text { L flux } \\
(\mathrm{Jy})\end{array}$ & $\begin{array}{c}\text { M flux } \\
(\mathrm{Jy})\end{array}$ & & \multicolumn{2}{c}{ Distance (pc) } & \\
\cline { 7 - 10 } & & & & & & $\mathrm{K}$ & $\mathrm{L}$ & $\mathrm{M}$ \\
\hline $10^{7}$ & 2225 & $\mathrm{M}$ & $3(-1)$ & $3(-2)$ & $3(-2)$ & 6000 & 300 & 200 \\
$10^{8}$ & 1437 & $\mathrm{~L}$ & $5(-3)$ & $1(-3)$ & $5(-3)$ & 700 & 50 & 70 \\
$10^{9}$ & 593 & $\mathrm{~T}$ & $1(-5)$ & $1(-5)$ & $5(-4)$ & 30 & 5 & 20 \\
\hline
\end{tabular}

Fluxes in Jy of a $15 \mathrm{M}_{\text {Jupiter }}$ object at $10 \mathrm{pc}$ distance, as a function of waveband $(\mathrm{K}, \mathrm{L}, \mathrm{M} \equiv 2.2,3.8$, and $4.6 \mu \mathrm{m}$, respectively) and age (adapted from Burrows et al. 2001). The effective surface temperature is also indicated, as is the spectral class, which passes from $M$ to $L$ to $T$ as the brown dwarf ages. The distance away that PILOT could detect such an object ( $5 \sigma, 1$ hour) is also indicated. The brown dwarfs themselves can be identified through their colours, though imaging through additional filters for on- and off- the methane bands, at 1.7 and $3.3 \mu \mathrm{m}$, would be required for accurate spectral classification.

is a survey project aimed at detecting old $\left(\sim 10^{9}\right.$ years $)$ brown dwarfs, though it is just one of many possible investigations of brown dwarfs that could be conducted. To detect all such brown dwarfs within a distance of $\sim 20 \mathrm{pc}$ in a $1 \mathrm{deg}^{2}$ survey area, as described in Table 5, would take $\sim 1$ month per filter (assuming a 25\% observing efficiency over that time). It is necessary to survey in at least three filters (K, L, M) and ideally also to obtain follow-up measurements in methane on-band and off-band filters (the methane bands are at 1.7 and $3.3 \mu \mathrm{m}$ ) to cleanly spectrally type the brown dwarfs. This project would therefore benefit greatly from the use of a dichroic beamsplitter and multiple arrays. Shallower surveys, covering greater areas of sky, could be conducted of younger (and hotter) brown dwarfs; for instance by spending 1 min per position instead of $1 \mathrm{~h}$, brown dwarfs of age $\sim 10^{7}$ years could be detected to distances of 2000,100 , and $60 \mathrm{pc}$, in the $\mathrm{K}-$, L-, and M-bands respectively, within a $50 \mathrm{deg}^{2}$ survey area.

PILOT's good near-IR image quality over wide fields of view will also allow it to make headway in the task of undertaking astrometry of nearby objects. Brown dwarfs are now regularly being discovered through several survey programs. They represent a 'linking class' of objects, between the relatively well-understood low-mass stars, and the (currently unobservable) extrasolar planets being inferred around nearby stars. A key measurement required to quantify the physical characteristics for these brown dwarfs is their distance, and the only way to obtain it is via astrometric measurements of their parallax.

With its wide-field of view (providing many background reference stars against which to measure positions) and image quality of a factor of five better than that available on current leading astrometric facilities (i.e. 0.2" versus $1^{\prime \prime}$ ), PILOT would be able to push the frontiers for high precision astrometry from the current 0.5 milliarcsecond per epoch limits down to 0.1 milli-arcsecond per epoch. This is equivalent to measuring distances five times further away, extending the reach from 50 to $250 \mathrm{pc}$. This is sufficient to measure a number of Southern star clusters, and so make possible a census of their brown dwarf populations.

\subsubsection{Studies of Pulsar Wind Nebulae (PWNe)}

A PWN represents the interaction of the electromagnetic wind from a young pulsar with the surrounding environment. Some $99 \%$ of the pulsar's spin-down energy is carried away in this electromagnetic wind, which is only seen through the shock features resulting from the interaction. One of the features seen is unequal bipolar jets, extending along the inferred pulsar spin axis. These jets often terminate in one or more bright knots of emission. Other features seen include fibrous arcs, or wisps as they are often called, which are cylindrically symmetric about the spin axis.

Furthermore, these features are dynamic. The Crab Pulsar Nebula is the prototype of an active PWN. The knots, wisps and jets have been observed (Hester et al. 2002) to vary on timescales of a month at both optical and $\mathrm{X}$-ray energies, and a good correspondence has been found between images in both energy ranges (with HST and Chandra). Faster variability, on timescales of days, has also been observed in the near-infrared by Melatos et al. (2005) using adaptive optics on Gemini. The latter images have allowed a good determination of the spectral slopes of the different features, and have provided evidence for different emission processes in the wisps compared to the knot at the base of the jet.

The most promising advantage offered by PILOT is the possibility of high-angular resolution imaging at optical wavelengths, allowing one to probe physical scales previously only achievable with $H S T$. An interesting possibility would be to examine variability of both the non-thermal shock-related features and the emission-line features, such as the $\mathrm{H} \alpha$ filaments in the surrounding environment.

Diffraction-limited imaging in the near-IR over a full field, without the distortions introduced by AO systems, would be desirable for this work. One of the problems encountered by Melatos et al. (2005) was good characterization of the changing point-spread function (PSF) over the AO-corrected field, a problem what would be mitigated somewhat by the large isoplanatic angle available at Dome C.

There are two approaches that could be taken for the observations. One would be to take high-resolution images 
through a range of filters, from the optical to near-IR, to determine the spectral shapes, and hence the emission processes. The second approach would be to look for variability in the features over a period of months. This would require imaging once per week (depending somewhat on the individual PWN being studied), for several months, building up a lightcurve and looking for movement of features. If done at near-IR wavelengths, however, the frequency may need to be higher, due to the potentially faster variability.

\subsubsection{Searching for Obscured AGB Stars in the Magellanic Clouds}

The chemical enrichment of the ISM is largely provided by mass loss from evolved asymptotic giant branch (AGB; i.e. post-helium burning) stars. Though many such stars have been identified in optical and near-infrared sky surveys, those with the highest mass loss rates ( $\geq 10^{-5} \mathrm{M}_{\odot} \mathrm{yr}^{-1}$ ) are often too obscured to be seen optically. Their spectra peak between 2 and $5 \mu \mathrm{m}$. In many ways these are the most interesting sources, yet because they are only bright in the infrared and in molecular lines, they remain a poorly studied population. This is especially the case in the Magellanic Clouds (MCs), due to their greater distance and the limited sensitivity and resolution of the IRAS satellite through which they have been identified. However, the well-constrained distances to the MCs, and the relative lack of Galactic foreground absorption or emission, provide a key advantage: stellar luminosities can be determined more accurately. This has been used, for example, to demonstrate differences in the periodluminosity relation between obscured and optically visible AGB stars (Wood 1998). In addition, the low metallicity of the MCs provides a unique environment in which to study the process of 'early' metal enrichment as would have occurred in the young Universe (see e.g. van Loon 2000).

Our knowledge of the AGB population in the LMC, based for many years principally on IRAS data, has improved significantly with the completion of the 2MASS and MSX surveys (Egan, van Dyk, \& Price 2001). The MSX A-band $(8 \mu \mathrm{m})$ provides better source positions and sensitivity than the IRAS $12 \mu \mathrm{m}$ band. Egan et al. (2001) showed that inclusion of the MSX data can lift the severe colour degeneracies, e.g. between planetary nebulae and HII regions, that are present in near-IR data from 2MASS. However, the sensitivity of MSX was limited to magnitudes brighter than 7.5, whereas a survey with PILOT could reach a limiting sensitivity of $14.8 \mathrm{mag}$ at $3.8 \mu \mathrm{m}, 13.4 \mathrm{mag}$ at $4.8 \mu \mathrm{m}$, and $7.6 \mathrm{mag}$ at $11.5 \mu \mathrm{m}$, over many square degrees (see Table 4), and deeper if just selected regions of the sky were chosen. This would be particularly valuable for identifying the dustenshrouded post-AGB stars that are the progenitors to planetary nebulae. These are expected to be quite rare due to the short duration $\left(\sim 10^{3}\right.$ years $)$ of this phase of stellar evolution, so well-calibrated data with $\lambda>3 \mu \mathrm{m}$ are essential to distinguish them from the far more numerous red giant population. Follow-up studies could include photometric monitoring to study their variability, spectroscopy to look for nebular emission lines, and infrared and (sub-)millimetre spectroscopy to study their dusty, molecule-rich envelopes.

Antarctica is the best place on earth to view the Magellanic Clouds. Around latitude $30^{\circ} \mathrm{S}$, where most southern telescopes are located, the MCs reach only moderate elevations of $40-50^{\circ}$, and then only in the summer if one wishes to observe at night. In Antarctica the MCs can be observed continuously at high elevation. There are other advantages that PILOT has for this work. The wide field helps to conduct blind surveys of the MCs for sources missed by IRAS. The high angular resolution helps to resolve sources in star clusters and crowded fields. The wide spectral coverage, from the visible into the thermal infrared, helps for detailed spectral typing.

\subsubsection{Stellar Populations and Near-Field Cosmology}

Our understanding of galaxy formation and evolution has largely rested to date on the measurement of global quantities like galaxy luminosity, mass, colour, and type. New measurements, however, involving deep optical and near infrared imaging of stellar populations in a few nearby galaxies, have begun to show that these global estimates hide a richer past.

In the Local Group, there are two dominant galaxies (the Milky Way and M31) and more than 40 (mostly dwarf) galaxies. The star formation histories of all dwarfs reveals an old stellar population, followed by a complex and chequered history. While there may be some dependence of the populations on the radial distance of a dwarf to the nearest large galaxy, existing data are not sufficient to provide strong support for such assertions.

For the Galaxy, we know a great deal. For instance, Figure 14 shows the age-metallicity distribution for all components of the Galaxy. This complex plot, however, defies any simple interpretation. The stellar bulge, halo, and thick disk are all dominated by old stellar components.

The deepest colour-magnitude diagram to date has come from the HST (Brown et al. 2003). A small patch of the outer M31 halo was imaged in two bands for a total of 100 orbits. The data reach down to $\mathrm{V}=31$ (with $50 \%$ completeness), well below the main sequence turn off at the distance of M31. These data clearly demonstrate the important contribution of an intermediate age (7-9 Gyr) population in M31, a stellar population not seen in the Galactic halo. This emphasizes the different accretion histories that are possible within two neighbouring galaxies.

Unfortunately, the HST is likely to be decommissioned before this important work can be completed. There is an opportunity for PILOT to fill this gap, through imaging galaxies in the local group in V- and K-bands to resolve their stellar populations. It is necessary to study a variety of galaxies in different Local Group density fields, in order to examine how the accretion histories depend on both the 


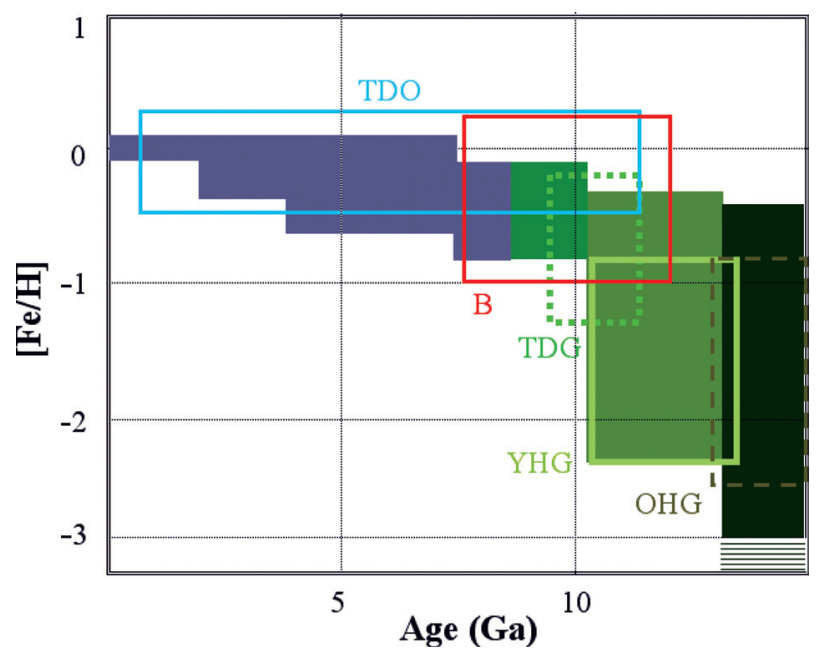

Figure 14 The age-metallicity relation of the Galaxy for its different component: $\mathrm{TDO}=$ thin disk open clusters; $\mathrm{TDG}=$ thick disk globular clusters; $\mathrm{B}=$ bulge; $\mathrm{YHG}=$ young halo globular clusters; $\mathrm{OHG}=$ old halo globular clusters. The blue corresponds to thin disk field stars, the green to thick disk field stars and the black shows the distribution of halo field stars extending down to $[\mathrm{Fe} / \mathrm{H}]=-5$. From Freeman \& Bland-Hawthorn (2002).

halo or the local density field. Around 20 galaxies should be studied, covering four different group density regimes.

Important inroads into this issue can be made out to $2 \mathrm{Mpc}$ with PILOT. In V-band, reaching to $29 \mathrm{mag}$, it is possible to extend below the main sequence turn off point for an old stellar population. Equivalently, it is necessary to reach $\mathrm{K}=25$ or $\mathrm{J}=25 \mathrm{mag}$. While the best colour baseline will include $\mathrm{V}$ and $\mathrm{K}$, the project can be tackled, to a lesser extent, through I-K colours, and even just J-K colours. The main source of confusion comes from signal fluctuations due to unresolved stars. A $0.2^{\prime \prime}$ PSF will be adequate for this project, fully sampled over $5^{\prime}$ fields of view. To go this deep in V-band, will require $\sim 100$ hours of integration to achieve a SNR $=5$. To go beyond the Local Group, however, for instance to reach the Virgo cluster, will require the successor telescope to PILOT.

\subsubsection{Stellar Streams and Dark Matter Halos}

A fundamental prediction of cold dark matter (CDM) cosmologies is that the dark matter halos of massive galaxies, like our own Milky Way, should be significantly flattened. The shape of the dark matter halo influences the motion of orbiting satellites, with asphericities introducing strong torques which act to precess the orbit. Until recently testing this has proven to be problematic due to the lack of suitable kinematic tracers beyond the Galactic disk.

The Sagittarius dwarf galaxy is being slowly dismembered by the tidal forces of the Milky Way. Throughout its demise, stars have been torn from the body of the dwarf and now litter the orbit, and this tidal stream of stars now completely encircles the Galaxy. With its extensive range through the dark matter halo, it was realized that the morphology and kinematics of this stream provided an ideal tracer of the underlying mass distribution of the halo. Several analyses of the stream, however, have concluded that the dark matter must be essentially spherical, at odds with theoretical expectation.

While not completely ruled out by CDM, the apparent spherical form of the halo of the Milky Way raises questions as to the nature of dark matter in general. Clearly, it is important to determine the shapes of the halos of other galaxies. While tidal streams tend to be extremely faint ( $\sim 29-30$ mag $\operatorname{arcsec}^{-2}$ ), and hence difficult to detect when unresolved, they stand out morphologically when considering the distribution of individual stars (Ibata et al. 2001; McConnachie et al. 2004). As pointed out in Section 6.2.11, PILOT can play an important role in the detection of resolved stellar populations within the Local Group. With a wide-field camera, it will allow an expansion of this program, allowing global stellar population properties to be determined (Ferguson et al. 2002). Mapping the halos of Local Group galaxies and nearby groups (such as the Sculptor Group) will clearly reveal any tidal streams associated with disrupting systems, calibrating the current rate of accretion. The morphology of any tidal streams will provide a measure of the mass and shape of the dark matter halo, allowing a catalogue of halo shapes to be determined.

To fully characterize the shape of the dark matter halo, the detailed orbital properties of a dwarf undergoing disruption need to be determined. To do so, the kinematic properties of the stellar streams are required. Current studies of the extensive stellar stream discovered in M31 reveal that, with the 10-m Keck telescope, stellar kinematics can be determined to an accuracy of $10 \mathrm{~km} \mathrm{~s}^{-1}$ for a star with $m_{I} \sim 21 \mathrm{mag}$ in a one-hour integration (Ibata et al. 2004). Since orbital velocities are of the order $250 \mathrm{~km} \mathrm{~s}^{-1}$, this velocity resolution is sufficient to accurately determine the kinematics of the stream. While PILOT may be too small to compete with Keck in this measurement, a larger Antarctic telescope equipped with a spectrometer would readily provide kinematic measurements of tidal stream stars in various galaxies, and hence place significant constraints on the shape of their dark matter halos.

This programme, to image at high resolution and sensitivity in the V-and K-bands the halos of galaxies, takes advantage of the high angular resolution and low sky background afforded by Antarctica. It requires imaging with $0.1^{\prime \prime}$ pixel scale, and a wide field in order to include the halos. It is focussed on Local Group galaxies; i.e. those within $\sim 3 \mathrm{Mpc}$ of the Sun.

\subsection{Our Universe and its Evolution}

\subsubsection{A Survey for Supernovae in Starbursts}

Core-collapse supernovae (CCSNe) are responsible for generating, then liberating, the bulk of the light metals in the Universe. As such, all models for the chemical evolution of galaxies have the CCSNe rate as a fundamental input parameter. Despite the number of amateur and robotic supernova searches now underway, these can only deliver a lower limit to the actual rate.

The CCSNe rate will of course be greatest in the regions where the star formation rate is high, i.e. starbursts, 
Luminous (LIRG), and Ultra-Luminous (ULIRG) Infrared Galaxies. Unfortunately, these regions are also heavily obscured by dust $\left(\mathrm{A}_{v}>10\right)$, and crowded with young star clusters, making early detection of CCSNe in these regions extremely difficult. Near-infrared, diffractionlimited observations with PILOT would yield the spatial resolution required $\left(0.2^{\prime \prime} \equiv 100 \mathrm{pc}\right.$ at $\left.100 \mathrm{Mpc}\right)$ to reveal $\sim 5$ new CCSNe per year (based on current models; Mattila \& Meikle 2001) against a crowded and dusty background in 15 LIRGs/ULIRGs, that would be missed by existing searches. The ability to re-visit each galaxy at any time during the four month winter increases the odds of a discovery, as well as enabling a light curve to be built up. Adaptive optics systems such as NAOS-Conica (NACO) on the ESO-VLT do not perform as well as had been hoped when guiding on the bright, but partly-resolved ULIRG nucleus, so truly diffraction-limited imaging with a 2-m telescope in Antarctica should be competitive with this facility.

Observations in the $\mathrm{K}$-band $(2.2 \mu \mathrm{m})$ are best suited for discovering, then confirming CCSNe, while $\mathrm{J}$ and $\mathrm{H}$ (1.25, $1.65 \mu \mathrm{m})$ images assist in determining the line-of-sight extinction and in confirming the type of supernova found. Observations are needed of 15 LIRGs/ULIRGs three times per year for 20 minutes each. The opportunity for discovery increases with the frequency of visits, as does the quality of the light curves and hence the accuracy of the sub-classification. Each CCSNe discovered allows us to probe the circumstellar medium, which contains a fossil record of the progenitor's mass-loss history and/or a binary companion, as in the case of SN 2001ig (Ryder et al. 2004).

\subsubsection{Time Delays in Gravitational Lenses}

One of the more elegant ways to measure the Hubble Constant, $H_{0}$, is to utilize strongly lensed quasars. When a quasar is lensed by intervening matter into two or more images, the distance that the light travels to form each image is different. This path length difference will translate into a time delay measured by the observer between the images. If the quasar shows significant photometric variations, the light curves for each image will be shifted relative to each another. One can obtain a measurement of $H_{0}$ since the time delay depends on the geometric distances to the lens and the source, which in turn depends on the value of $H_{0}$. Such a measurement of $H_{0}$ is independent of systematics found in determinations that use the 'cosmic distance ladder'. Recent reviews of the topic can be found in Kochanek \& Schechter (2004) and Kochanek (2002).

In practice, the measurements are more complicated than simply measuring a time delay. It is also necessary to obtain good astrometry of the lensed images, and good quality imaging of the entire system. The astrometry is required to determine by how much the images have been deflected. Good quality imaging is important for the construction of the lens model, to constrain the nature of the lensing object (i.e. is it a galaxy or a cluster?), and to determine the location of the lensed images. Images with good spatial resolution are thus crucial for accurate measurements. Such studies are currently done with ground-based telescopes but often require some degree of deconvolution, which carries with it potential systematic effects.

The evaluation of $H_{0}$ from gravitational lenses allows comparison of measurements made in the local universe, such as the value of $H_{0}=72 \pm 8 \mathrm{~km} \mathrm{~s}^{-1} \mathrm{Mpc}^{-1}$ from the HST Key Project on the extragalactic distance scale (Freedman et al. 2001). Recent lens determinations yield a similar value $\left(71 \pm 3 \mathrm{~km} \mathrm{~s}^{-1} \mathrm{Mpc}^{-1}\right.$; Kochanek \& Schechter 2004) only under the assumption that the lensing galaxies have a constant mass-to-light ratio. The more likely case (based on theory and other observations), of an isothermal density profile in these galaxies, results in a significantly lower value of $48 \pm 3 \mathrm{~km} \mathrm{~s}^{-1} \mathrm{Mpc}^{-1}$, raising the possibility that local determinations of $H_{0}$ are too high. These results are based on just four systems, however, so more lensed systems need to be investigated. A telescope such as PILOT provides an opportunity to address this problem, and offers two important advantages for observing programs over other telescopes:

1. High spatial resolution at optical and near-infrared wavelengths: Since PILOT will provide near-diffraction limited images from the optical to the near-IR, the performance makes it comparable to the HST. Good angular resolution will enable accurate modelling of the lens system, crucial for correct interpretation of the time-delays and a measurement of $H_{0}$.

Diffraction-limited imaging in the near-infrared is a niche not being exploited elsewhere. As well as high angular resolution, K-band $(2.2 \mu \mathrm{m})$ imaging will be important for studies of dusty, reddened, lensed systems, where extinction in the lensing galaxy makes the quasar images fainter, increasing the photometric errors for a given exposure time. Additionally, near-infrared light is more representative of the total stellar mass of a galaxy, so K-band imaging of the lens galaxy will allow better mass density determination.

2. Ability to monitor continuously for long periods: For good determination of the time-delay, good temporal sampling is necessary, particularly when the timescale of photometric variations is not known, a priori. Furthermore, the time-scale can range from days to months, and so a long baseline may well be needed to determine the delays.

A typical observing scheme that can be envisaged would be a 'snapshot'-style program of relatively short exposures, repeated regularly to build up a light curve. These exposures could thus fit into other scheduled programs being executed on the telescope. An example program would be an image every $12-24 \mathrm{~h}$, over the winter. This would build up a dense lightcurve that would enable a quite precise time-delay measurement.

Known quasar lenses are rarely more than a few arcseconds in size (given by the separation of the images), 
but the lensing system will often be larger. The field of view of the strawman AO-corrected optical imager, or the high-resolution near-IR imager, will be sufficient for these purposes.

\subsubsection{Galaxy Evolution: a Deep Near-IR Extra-Galactic Survey}

This program addresses three key areas studied extensively with recent HST deep field observations, through a wide-field near-IR survey:

1. I-band drop out galaxies at $\mathrm{z}>6$ : With the release of the HST ultra-deep field (UDF), attention has focused on the possibility of $z^{\prime}$ band drop outs and higher redshift objects. However, such work requires IR photometry in order for candidates to 'drop out' of the $z^{\prime}$ band. The scarcity of such objects requires wider field observations than are practical with HST/NICMOS or 8-m class telescopes. Using the PILOT near-IR camera it would be possible to take this technique to the next level, with observations in the ZYJHK-bands replacing the classical Lyman break selection using UBVRI at lower redshifts $(z<4)$. The wide field of PILOT makes this project competitive in this field with an investment of some tens of hours of telescope time.

2. New classes of object: Dickinson et al. (2000) discovered a quite extraordinary objects (HDF-N $\mathrm{J} 123656.3+621322$ ) in the NICMOS observations of the HDF-N. This object was detected only in the NICMOS H-band image and then in follow-up groundbased K-band imaging. Recently, Yan \& Windhorst (2004) reported several similar extremely red objects in the NICMOS UDF image. However, their nature and space density is currently unknown, with plausible explanations ranging from $z>10$ galaxies to extreme carbon stars. A wide field survey will make it possible to address problems associated with small number statistics and define a plausible spectral energy distribution for such types of object.

A third program, studying galaxy evolution by examining the morphology of galaxies at $z>1$ through their rest frame optical light red-shifted into the near-IR, is described in more detail in Section 6.3.6.

A 2-m telescope at Dome C offers a critical combination of depth and area for these survey projects. The deep optical HST surveys (HDF-N/S, GOODS, UDF) have shown what can be achieved with excellent quality data. The near-IR surveys undertaken with the HST/NICMOS camera, however, are limited in their angular coverage. PILOT, however, could deliver images to depths and resolutions on a par with NICMOS, over fields of view that are far larger.

Unfortunately, the southerly location means that PILOT will struggle to observe the southern HST GOODS field, centered on the Chandra Deep Field South. However, the HDF-S is well placed for study, providing excellent optical data to use in tandem with the PILOT observations.
One could envisage a series of survey projects, with different depths and area coverage, to address complementary science goals. An excellent model for such a survey would be the three planned UKIRT extragalactic near-IR surveys, which provide a sequence of increasing depth for smaller areal coverage (namely, the UKIDSS Large Area Survey (LAS), Deep Extragalactic Survey (DXS) and Ultra Deep Survey $\left.(\mathrm{UDS})^{8}\right)$. The key opening for PILOT is improved spatial resolution, which will uniquely allow morphological studies, for a large sample of galaxies, in the rest frame optical bands over the redshift range $z=1-3$.

To tackle such projects requires the use of large-format near-IR cameras, with a $0.1^{\prime \prime}$ pixel scale to adequately sample the PSF at the shortest wavelengths. The filter set should include ZYJH and K. With a field of view of $5^{\prime}$, this would be well matched to the HST deep fields. With a ten hour exposure a sensitivity of $22.3 \mathrm{mag} \operatorname{arcsec}^{-2}$ would readily be obtained in K-band, on a par with those of the NICMOS HDF-S surveys. The resolution and area combination will allow ground breaking studies. The basic program therefore requires $50 \mathrm{~h}$ of observation $(10 \mathrm{~h}$ in each of five filters) on one field.

\subsubsection{Emission-Line Mapping of the High Redshift Universe}

While classical imaging and long-slit spectroscopy have provided a wealth of astronomical data, our understanding of the detailed properties of objects in the high redshift universe requires spatially resolved spectroscopy. The good seeing and low sky background available in Antarctica immediately lends itself to integral field spectroscopy. Two potential astrophysical studies include:

1. Gravitational lensing: Massive galaxies can split the light from distant quasars into a number of images, separated by $0.5-1^{\prime \prime}$ on the sky. As well as producing multiple images, these gravitational lenses can also induce significant magnification, producing gross distorted images of normally unresolved features, such as the Broad Emission Line Region (BLR; Mediavilla et al. 1998; Motta et al. 2004). With gravitational lens inversion, the fine detail of such emission regions can be exquisitely mapped (e.g. see Wayth et al. 2005). Furthermore, additional differential magnification effects are expected to occur, resulting in differing variability for continuum and emission sources (Lewis et al. 1998). For such a study, spectroscopic monitoring is required, but it is also important for the emission from various lens images to be clearly separated. Hence, an integral field unit taking advantage of the good seeing afforded by an Antarctic telescope is ideal.

2. Star-forming galaxies: In recent years, the submillimetre/infrared view of the high-redshift universe has revealed that much of the star formation in young systems is hidden, buried in dust cocoons that re-radiate

\footnotetext{
${ }^{8}$ www . ukidss . org
} 
the intense UV produced by massive young stars at much longer wavelengths. Sub-millimetre imaging has uncovered a substantial population of these galaxies at at redshifts of $z>1$, and integral field spectroscopy of the brightest examples have revealed complex structure and dynamics, interpreted as violent interactions and star burst induced superwinds (e.g. Swinbank et al. 2005). Furthermore, similar star forming populations in the early universe have now been uncovered by the space infrared telescope Spitzer. Integral field spectroscopy of these galaxies will be vital in uncovering the physical properties driving star formation in these young systems.

Of these two studies, gravitational lens systems provide a simpler observational challenge, requiring integration times of order one hour with current integral field spectrographs on 4-m class telescopes in order to make detailed maps. The high angular resolution, however, is needed to ensure that the resultant continuum subtracted images reflect real emission features, rather than poor PSF subtraction. The study of star forming galaxies is more technically challenging, with Ly $\alpha$ imaging of the brighter Spitzer sources requiring $8 \mathrm{~h}$ on current 8-m class telescopes. Both Ly $\alpha$ and $\mathrm{H} \beta$ imaging would take advantage of the significantly lower sky background in Antarctica, so bringing these projects into the grasp of PILOT.

To conduct this program requires using a lenslet array with $0.1^{\prime \prime}$ spatial resolution. Widths of typical lines are from 500 to $5000 \mathrm{~km} \mathrm{~s}^{-1}$, targeting Ly $\alpha$ at $z>2.2$ $(\lambda>3900 \AA$ ) or $\mathrm{H} \beta$ in the near-IR (for $z>1$ ).

\subsubsection{The Complete Star Formation History of the Early Universe}

The rise of the Universal star-formation rate (SFR) from $z=0$ to $z=1$ is well established from a variety of indicators (ultraviolet, Balmer lines, far-IR emission). The history of the Universal SFR for $z>1$ is poorly determined; it appears to peak and decline from $z=1$ to $z=6$ (the infamous 'Madau-Lilly diagram'; Madau et al. 1996; Lilly et al. 1996). However this result comes from the measured ultraviolet light of high-redshift star-forming galaxies ('Lyman Break Galaxies' or LBGs) and there is a big problem: dust (see e.g. Steidel et al. 1999; Glazebrook et al. 1999).

Interstellar grains are thick in star-forming regions and are very effective at absorbing ultraviolet (UV) light from young stars. For example, typical visual extinctions observed in star-forming galaxies locally predict large extinctions ( $>2 \mathrm{mag}$ ) in the UV. In fact, there is now extensive evidence that this is also true at high-redshift. The slopes of the UV spectra of LBGs are consistent with around $2 \mathrm{mag}$ of extinction. There is also a population of sub-millimetre sources (the 'SCUBA galaxies') which can only be explained by very dusty starbursts. Typically when plotting a SFR versus $z$ diagram constant extinction is assumed for lack of anything better. The majority, 90\%, of the UV light is absorbed.
Paradoxically, it turns out that the best place to measure the SFR of a galaxy is in the deep red. This is because this is where the $\mathrm{H} \alpha$ emission line comes out. The $\mathrm{H} \alpha$ line strength is a direct measure of the number of ionizing photons in a galaxy and hence the number of young stars. Being emitted at $6563 \AA$ it is relatively little affected by dust, compared to Ly $\alpha$, with a mean $\mathrm{A}_{v} \sim 1$ mag through the galaxies. At $z>1 \mathrm{H} \alpha$ is observed in the near-IR, and some limited spectroscopy has been obtained from $z=1$ galaxies (Glazebrook et al. 1999; Doherty et al. 2004) and $z=3$ LBGs (Erb et al. 2003). These typically show the SFRs to be several times larger than was calculated from the UV light, consistent with most of it being absorbed by dust. However, only a handful of galaxies have been studied due to the extreme difficulty of obtaining near-IR spectroscopy, even on 8-m class telescopes.

To truly measure the evolution of the cosmological SFR we need deep, $\mathrm{H} \alpha$-selected samples to allow the construction of the $\mathrm{H} \alpha$ luminosity function from $z=1$ to $z=4$. The only existing dataset which comes close to doing this is from HST, using the NICMOS camera in a slitless grism mode in $\mathrm{J} \& \mathrm{H}$. Because of the low space background, slitless images can be used to directly search for lines down to a limiting $\mathrm{H} \alpha$ flux. The resulting $\mathrm{H} \alpha$ luminosity function has been measured at $z=1.5$ (Yan et al. 1999; Hopkins, Connolly, \& Szalay 2000) for only a few dozens of objects over just tens of square arcminutes.

The 'cosmologically dark' K-band window from PILOT would allow us to extend this work to high-redshift and backwards in time - in particular to $z=3$ and the era of Lyman break galaxies. Deep imaging in the K-band at a series of redshifts from $z=2.0$ to $z=3.0$ with narrow band filters is required. In conjunction with deep broad $\mathrm{K}$ images, narrow-line excess objects can be identified using standard techniques.

Is star-formation really declining from $z=1$ to $z=3$ ? Such a long baseline (2-6 Gyr after the Big Bang) will reveal the epoch of peak galaxy formation. The measured SFR-distribution function will probe how it depends on galaxy size, and when combined with the deep broad-K observations discussed in Section 6.3.6, on galaxy mass. In fact, the combination of direct SFR measurements and stellar mass measures of the same galaxies has proved powerful at $1<z<2$ in the Gemini Deep Deep Survey direct evidence for 'downsizing' (the motion of peak SFR to smaller mass galaxies with time) is seen (Juneau et al. 2005). Both these measurements at $z>2$ are ideally suited for deep K-band observations.

This program requires narrow-band observations which are most sensitive in the darkest windows; i.e. the $\mathrm{K}$-dark window is ideal and confers a considerable advantage for this type of work. Diffraction limited imaging $\left(0.3^{\prime \prime}\right)$ over a wide field, only possible from Antarctica, makes it possible to search for extremely compact dwarf star-forming galaxies at these redshifts. The number of galaxies found is the determining factor for luminosity function calculations, and so the uniquely wide field is an advantage. 
Narrow band filters, of $1 \%$ bandwidth, will be required from 2 to $2.6 \mu \mathrm{m}$. A $10 \mathrm{~h}$ exposure through a $1 \%$ filter at $2.2 \mu \mathrm{m}$ will reach a line flux of $10^{-17} \mathrm{ergs} \mathrm{cm}^{-2} \mathrm{~s}^{-1}$ for PILOT parameters. At $z=2.35$ this is $\mathrm{L}(\mathrm{H} \alpha)=$ $4 \times 10^{41} \mathrm{ergs} \mathrm{s}^{-1}$, or a SFR of $2.8 \mathrm{M}_{\odot}$ per year, an exceptionally low level to be sensitive to.

The line limit corresponds to an equivalent width of $20 \AA$ in the rest frame if we match the galaxies detected through their emission lines in the narrow band filter to those that would be seen in a deep (i.e. $100 \mathrm{~h}$, $\mathrm{K}<23.5 \mathrm{mag}$ ) broad band image (as discussed in Section 6.3.6). This would result in complete emission line identification of the K-selected sample (the limit would detect $80 \%$ of star-forming galaxies locally and even more when extrapolated to high redshift) with corresponding unambiguous photometric redshift identification from the line detections + broad-band colour constraints. Comparing the measurements of stellar mass and SFR would allow us to study the 'downsizing' of galaxy assembly at $z>2$ when the most massive ones form.

The number of objects that would be found can be estimated using the luminosity function of Hopkins et al. (2000) and transposing it to $z=2.35$ (i.e. with no evolution). For $S>10^{-17} \mathrm{ergs} \mathrm{cm}^{-2} \mathrm{~s}^{-1}$ we calculate a $\mathrm{H} \alpha$ source density of 300 per $20^{\prime}$ diameter PILOT field, an ample number for eliminating cosmic variance which plagues smaller surveys. (At $z=2.35$ the survey size is $\sim 40 \times 35 \times 35$ comoving Mpc, which is many clustering scales.)

One might also want to cover a larger area $(3 \times 3$ pointing) grid with shallower surveys as in the 'galaxy stellar mass' survey discussed in Section 6.3.6. This two tier approach would better sample rare, brighter objects as well as the more common, fainter ones.

With such deep narrow-band observations there might be many unidentified lines, some with objects too faint for reliable photometric redshifts and some which only appear in the line (i.e. no continuum). These would be excellent candidates for follow-up multi-object spectroscopy - the flux limit of $10^{-17} \mathrm{ergs} \mathrm{cm}^{-2} \mathrm{~s}^{-1}$ is easily in reach of telescopes such as Gemini with forthcoming new instruments (a typical calculation shows $\mathrm{S} / \mathrm{N}=5$ at the limit in $10 \mathrm{~h}$ ). An Antarctic ELT would do considerably better. Spectroscopy at moderate resolution $(\mathrm{R}=3000)$ could distinguish between various possibilities: one would observe $\mathrm{H} \alpha+[\mathrm{NII}]$ or $\mathrm{H} \beta+[\mathrm{OIII}]$ or the [OII] doublet or the asymmetric Ly $\alpha$ line (at $z=17$ ). Spectroscopy with wide wavelength coverage could directly measure the dust extinction (from $\mathrm{H} \alpha / \mathrm{H} \beta$ ) and metallicity (via R23 or O3N2 indices) of the $z>2$ star-forming gas.

Finally we can speculate on the serendipitous discovery of $z=17$ Ly $\alpha$ emission line galaxies. Is the possibility even reasonable? The serendipitous potential of really high-redshift searches $(z>10)$ from any deep K-band observations is high. It is unexplored parameter space. The Ly $\alpha$ luminosity at $z=17$ would be $>4 \times 10^{43}$ ergs $\mathrm{s}^{-1}$, which is a few times that of the $z=6.5$ object that Rhoads et al. (2004) found in a narrow band optical search in a survey of comparable sky area. Of course the SFR at $z>10$ is unknown, so its measurement is a powerful test of the hypothetical Population III and re-ionization. One point to bear in mind is that low-metallicity Population III galaxies would have much higher Ly $\alpha$ equivalent widths than their low-z counterparts. One might want to pursue this with a deeper narrow-band survey of $>100$ h exposure.

\subsubsection{The Evolution of Galaxy Mass and Morphology: High Resolution Imaging Beyond the Hubble Limit - a PILOT Ultra Deep Field}

The HST has revolutionized our view of the highredshift Universe with its deep, high-resolution images. Galaxies paint the sky in numbers up to $10^{7}$ per square degree and star-formation rates are much higher than they are today. The picture of the $1<z<4$ Universe is one of great disturbance: many anomalous looking galaxies abound with little sign of the regular Hubble sequence.

However, HST Deep Fields (HDFs) are inherently biased: They are predominantly taken in the optical and have very tiny fields of view of only a few $\operatorname{arcmin}$. At $z>1$ the optical samples the rest-frame ultraviolet — in such pictures only the scattered star-forming regions containing young UV luminous OB stars can be seen. Young 'Lymanbreak galaxies' (LBGs) with prodigious star-formations rates dominate the picture. Older, redder more regular stellar components cannot be seen. HST does have J- and $\mathrm{H}$-band imaging with the NICMOS camera, but here the field is even smaller and so no large surveys are possible.

To truly characterize the high-redshift Universe ultradeep surveys are needed in the near-infrared, ideally in the reddest possible band to pick up more normal galaxies at high-redshift. It is also necessary to cover much larger areas - the typical cosmic variance on the scale of the Hubble Deep Fields is $100 \%$, leading to large uncertainties in measurements.

Tantalizing results from deep ground-based near-IR surveys have revealed populations of massive galaxies beyond $z>1$ with much more regular morphologies. The Gemini Deep Deep Survey has shown spectroscopically that massive, old galaxies exist to $z=2$ at $\mathrm{K} \sim 20$ (GDDS; Glazebrook et al. 2004), and images from the ACS instrument on the HST reveal regular elliptical and spiral galaxies. Franx et al. (2003) have found a substantial population of 'Distant Red Galaxies' (DRGs) with J-K > 2.3, $z>2$ and $\mathrm{K}<22.5$; these appear to be massive and much redder/less UV luminous than LBGs. One has an HST NICMOS image showing a classical bulge plus disk.

Why is the near-IR so important? This is where the bulk of the light from old stars come out, and so to weigh a galaxy by stellar mass it is necessary to measure the light at wavelengths greater than that of the $4000 \AA$ break. For $z>2$ this means that the K-band is absolutely essential to measure stellar mass at high-redshift, rather than the transient UV bright episodes of star-formation. Massive galaxies tend to be highly clustered as well; there are zero 
at $z>2$ in the HDF-N and 3 in the HDF-S (as determined from deep VLT K band imaging) for example.

Ultra-deep, wide-area K-band imaging will obtain resolved images over substantial areas. The goal is to image large numbers of $z>2$ red galaxies and measure their morphologies. Are they ancestors to spiral galaxies? Do they have disks? How far back in time can we see disks or genuine elliptical galaxies? Such galaxies are the most interesting; galaxies which are already old and at $z=3-4$ constrain the epoch of first star-formation in the Universe, pushing it back to $z>>6$.

Quantitative morphological measures (bulge/disk decomposition, concentration, asymmetry, 'Gini' coefficients) will be applied. Comparison with deep optical images will establish photometric redshifts. Stellar mass functions will be established as a function of redshift and morphology, which will measure the nature of the growth of galaxies ('hierarchical assembly' or 'down-sizing'?) and hence test galaxy formation models. By covering large areas we will be able to measure clustering and hence obtain constraints on the dark mass of their halos.

This project takes advantage of the 'cosmological window' at $2.4 \mu \mathrm{m}$, where the sky is very dark, to enable ultra deep fields to be obtained. It also takes advantage of the wide diffraction-limited field offered by Dome C. The southern latitude means most of the southern sky is circumpolar and visible all winter, there is no daylight to interrupt observing. This is ideal for the accumulation of long exposures on deep fields. PILOT is the only facility conceived which can go to the required depth, over a large enough area.

Wide-field, K-band imaging, with a resolution of $0.3^{\prime \prime}$ or better, is required. $\mathrm{J}$ and $\mathrm{H}$ images are also desirable and perhaps $\mathrm{V}$ and I images (though these images could be obtained elsewhere, albeit not in as good seeing).

To study rare bright objects as well as more common fainter objects a two-tier approach is needed:

1. A $100 \mathrm{~h}$ exposure will reach $\mathrm{K}=23.5 \mathrm{mag}$ for extended sources, thus $\mathrm{K}<22.5$ mag galaxies will have $\mathrm{S} / \mathrm{N}>25$, suitable for determining the morphology of resolved objects with $0.3^{\prime \prime}$ resolution. A $20^{\prime}$ diameter FOV will contain 1000 galaxies of the type studied by GDDS and Franx et al. and an unknown number of fainter objects yet to be studied. The field would represent a 100 fold improvement in cosmological volume on any comparable large telescope imaging (e.g. the FIRES VLT field).

2. A $10 \mathrm{~h}$ exposure reaches $\mathrm{K}=22.3$; a $3 \times 3$ pattern of such fields will cover $1^{\circ} \times 1^{\circ}$ on the sky. A $1^{\circ}$ scale corresponds to a transverse size of 110 comoving Mpc at $z=3$, this field will be ample for studying the clustering and large-scale structure of $1<z<5$ red galaxies (with correlation lengths $\sim 10 \mathrm{Mpc}$ ).

The brightest galaxies ( $\mathrm{K}<21 \mathrm{mag}$ ) found at high-redshift would be followed up spectroscopically with Gemini (FLAMINGOS-2, GNIRS) to study stellar populations and velocity dispersions. Fainter galaxies could one day be followed up by JWST.
Such a uniquely wide-area deep K-band survey has the potential to detect new populations of objects at very high redshift $(z>13)$ which are invisible at shorter wavelengths. There is quite possibly a second epoch of star-formation in Population III objects at these redshifts (see Section 6.3.7). If this is the case then the number of galaxies that would be bright enough to be seen is unknown. The $\mathrm{K}=23.5 \mathrm{mag}$ limit corresponds to a rest frame unobscured UV luminosity from a SFR of $50 \mathrm{M}_{\odot}$ per year at $z=15$ and would be a factor two to three times more luminous than the brightest LBGs at $z=3$. Of course, the abundance of $z=15$ objects is a subject of educated speculation; for example these UV luminosities assume a Salpeter IMF, and a Population III IMF would result in even more UV output. These deep fields would lay the ground work for the cosmological science case for any future Antarctic ELT or future space telescopes such as JWST.

\subsubsection{Probing the First Light in the Universe with Gamma Ray Bursts}

Gamma Ray Bursts (GRBs) are the most powerful, energetic explosions in the Universe. For a period of a few days they are 100-1000 times more luminous than Quasars. Current satellite missions are capable of detecting the gamma ray flux of GRBs to $z=20$ and the SWIFT mission (launched in 2004) will be able to reach $z=70$ (Lamb \& Reichart 2001). Note that $z=20$ is $180 \mathrm{Myr}$ after the Big Bang (just over $1 \%$ of the current age of the Universe), $z=70$ is $28 \mathrm{Myr}(0.2 \%$ of now).

We now know that GRBs are associated with starformation in galaxies. They occur in off-nuclear starforming disks. Spectra have been obtained to redshifts as high as $z \sim 4$. The best theoretical model is that GRBs represent a 'hypernova' associated with the core-collapse of a super-massive star directly in to a black hole.

However $20-40 \%$ of GRBs are 'dark bursts'; i.e. they have no optical counterpart. Given that GRBs are detectable to very high-redshifts the natural conclusion is these dark bursts have $z>7$. At these redshifts all optical light ( $\lambda<\operatorname{Ly} \alpha$ in the rest frame) is removed by neutral hydrogen absorption in the IGM.

This would imply that a considerable amount of starformation occurred in the Universe at $z>7$, an epoch not yet probed by any observations, whether from ground or space. This would address a fundamental problem in cosmology: the re-ionization of the early Universe.

After its early fireball phase, the Universe consisted of neutral hydrogen until the first stars were formed at some, currently unknown, time. This is often referred to as the time of 'First Light'. These early stars would have produced ultraviolet radiation which would then ionize the Universe, some time between $z=20$ and $z=7$. Observations of galaxies and quasars at $z=6$ show that the amount of ultraviolet produced at this 'late' time is insufficient to ionize the Universe and further, that the Universe is already almost completely ionized so that this event must have 
happened earlier. Observations of the Cosmic Microwave Background are consistent with a range of $z=7-20$.

The early universe contained no heavy elements these are made in stars. Calculations of the likely modes of star-formation in pristine material shows that the very first generation of stars was likely to be have been very different from current day star formation, which occurs in a 'dirty' interstellar medium. The first stars are expected to be much more massive, on average, than stars born today. They are known as 'Population III' stars. Being massive, they would produce considerable ultraviolet radiation and hence be capable of ionizing the Universe. Many models predict an early peak of star-formation due to this Population III at $z>10$ (e.g. Cen 2003).

Because GRBs are ultra-luminous and trace massive star-formation, they can be used to trace Population III stars and re-ionisation. The opportunity is timely with the imminent launch of SWIFT, which will be rapidly followed by further, more powerful, gamma-ray satellites.

PILOT can provide rapid JHKLM imaging of GRB locations to search for afterglows; the position of the break will be revealed by colours and hence the redshift can be determined. Redshifts at $z>20$ can be probed. The M-band can reach $z=35$ if star-formation ever occurred back then - only 80 Myr after the Big Bang. Studying the redshift distribution of GRBs will reveal any Population III stars and the epoch of 'First Light'.

The L- and M-bands can be superbly probed at cosmological distances from Antarctica, where the sky is uniquely dark. The K-band is also enormously more sensitive in the Antarctic than from temperate sites, providing a window on $10<z<15$ GRBs. In other words, the regime where the Antarctic has its greatest advantage is exactly the redshift range that needs to be probed. GRBs are also point sources, which means the good seeing is a major advantage as it provides much better sensitivity.

The observations need rapid response, which rules out space telescopes (such as Spitzer and JWST) and even large conventional ground-based telescopes. The latter are not well suited to following up large numbers of events. In contrast PILOT could follow-up hundreds. The long polar night means any rapid response is not going to be interrupted by any untimely daylight-GRB light-curves could be followed continuously. Thus, PILOT is a uniquely capable probe of the $z>20 \mathrm{GRB}$ regime due to its singular combination of sensitivity and availability for frequent rapid response.

This project needs a $1^{\prime}$ field of view and K-, L-, and M-bands imaging capability; J- and H-band imaging, in addition, would be useful (SWIFT will produce 10" accuracy in positions, at the rate of about 100-200 per year, so that $10-80$ would have $z>7$ ). If the FOV was as large as $10^{\prime}$, it would be possible to work with the $\pm 4^{\prime}$ SWIFT $\gamma$-ray burst alert positions - it would be possible to follow-up GRBs with weak or absent X-ray afterglows which has never been done before.

Gamma ray bursts remain bright to very high-redshifts. Lamb \& Reichart (2001) calculate $\mathrm{L}=\mathrm{M}=15 \mu \mathrm{Jy}$ for a $z>20 \mathrm{GRB}$. One beautiful reason for this is that time dilation makes the fading slower at high-redshift, so if one observes at a fixed time after the event, the time dilation effect approximately cancels the cosmological dimming.

The Lamb and Reichart number above refers to $24 \mathrm{~h}$ post-burst. A typical afterglow fades at $t^{-4 / 3}$, so one hour after the burst these would be 70 times brighter (i.e. $\sim 1000 \mu \mathrm{Jy}$ ).

These fluxes are easily detectable with PILOT. A 5 minute exposure reaches a noise limit of $300 \mu \mathrm{Jy}$ in L; if it is not detected a 1 hour M-band exposure would reach $200 \mu \mathrm{Jy}$. This would correspond to a $z=36$ object. For the 'easy' $z=10$ case, we estimate that the GRB would be $700 \mu \mathrm{Jy}$ in K-band, whereas a 1 minute exposure in this band reaches to $15 \mu \mathrm{Jy}$. A filter sequence, JHKLM, would provide the desired combination of observations for find the GRBs.

We note there is considerable variation in GRB properties - many would be a lot brighter but some would be fainter. Part of the science objective is to pin down in detail their elusive properties.

An obvious upgrade would be a spectrograph working in the K- and L-bands. This will allow one to probe the gas in the Universe and the host galaxies at $z>10$. It would be possible to measure the elemental abundances, the largescale structure of the early Universe from metal forest absorption lines, and the amount of re-ionization from the shape of the Ly $\alpha$ edge.

Spectroscopy at $\mathrm{R}=100-300$ would be possible even on a 2-m telescope. Such a telescope is also an obvious pathfinder for an Antarctic ELT, which would be capable of making very high spectral and spatial resolution observations of Galaxies at $z>10$.

\subsubsection{Cosmic Shear}

Light rays follow geodesics, which bend in the presence of matter. It follows that a coherent shape distortion is imprinted in the distribution of distant background galaxies by mass fluctuations in the intervening cosmic web. This pattern of cosmic shear is a powerful cosmological probe. It is directly sensitive to the dark matter distribution predicted by theory, and does not depend on the details of how galaxy light traces mass.

The most serious limitation for ground-based optical cosmic shear experiments is the systematic variation in the PSF which arises from inevitable changes in atmospheric seeing and telescope properties with position and time (e.g. Kaiser, Squires, \& Broadhurst 1995). These PSF distortions are typically an order of magnitude larger than the cosmological shear that we wish to measure. The outstanding natural seeing performance (i.e. stable atmosphere) obtainable at Dome $\mathrm{C}$ is therefore advantageous for controlling systematic PSF variability and, equally importantly, resolving high-redshift galaxy shapes.

In addition to excellent image quality, cosmic shear surveys demand high galaxy surface densities (10$100 \mathrm{arcmin}^{-2}$ ) to reduce statistical noise. This requirement is much more important than measuring accurate 
shapes for individual galaxies, because there is an irreducible experimental scatter in the shape information owing to the unknown galaxy ellipticity before shear. Galaxy shapes must be resolved, but are not required to be measured at high signal-to-noise.

Cosmic shear experiments may be broadly divided into two categories (for useful reviews see Hoekstra, Yee, \& Gladders 2002; Refregier 2003):

\section{Experiments mapping 'blank fields' to detect the shear} pattern resulting from gravitational lensing by the cosmic web of large-scale structure: Current state-ofthe-art surveys cover a few square degrees and have measured the amplitude of mass fluctuations (denoted $\sigma_{8}$, where the subscript refers to a scale of $8 \mathrm{Mpc}$ ) to $10 \%$ (e.g. Bacon et al. 2003). In 10-15 years, cosmic shear surveys will cover the whole sky and will have the power to characterize the properties of the dark energy and matter as a function of redshift.

2. Experiments targeting the most massive clusters where the shear distortions are the largest: The shear pattern can be used to reconstruct the cluster gravitational potential (Kaiser \& Squires 1993), that can be compared with CDM (cold dark matter) theory. Currently this has been performed for tens of clusters and also from space using the $H S T$.

The required magnitude limits in optical or near-infrared wavebands to deliver the minimum source density of 10 $\operatorname{arcmin}^{-2}$ (of galaxies that are usable for lensing studies) are $\mathrm{R} \sim 24$ or $\mathrm{K} \sim 21 \mathrm{mag}$. To date, most cosmic shear experiments have been performed in the optical — with existing facilities, the attainable surface density of background galaxies (in a fixed integration time) is roughly an order of magnitude higher with optical observations than with near-IR imaging. Moreover, advances in infrared detector technology have lagged significantly in terms of the available field-of-view.

However, given the greatly reduced near-IR background available in Antarctica, it is timely to reassess this situation. Mapping cosmic shear in infrared wavebands offers a number of advantages: (1) galaxy shapes are smoother, tracing older stellar populations rather than knots of star formation, thus ellipticities are easier to measure; (2) background galaxies at high redshift $(z>1)$ are more readily detected; (3) the availability of infrared colours greatly enhances the efficacy of photometric redshifts, allowing a more accurate determination of the redshift distribution of the background galaxies; (4) for observations targeting cosmic shear behind galaxy clusters, the availability of K-band imaging enables the 'contamination' from foreground objects and cluster members to be greatly reduced (e.g. King et al. 2002); and (5) combining the K-band luminosity of such clusters with the weak lensing mass estimate yields a mass-to-light ratio.

Let us assess the competitiveness of some specific cosmic shear observational programmes possible

\section{with PILOT:}

- 'Blank-field' cosmic shear survey over 100 square degrees: This would be competitive if completed within $\sim 5$ years (the CFHT Legacy 'wide' survey is intending to map $\approx 170$ sq. deg.). PILOT can reach the required detection sensitivity of $\mathrm{K} \approx 21$ in a $\sim 1$ hour pointing; the $20^{\prime}$ field-of-view of the near-IR wide-field imager implies that $100 \mathrm{deg}^{2}$ can be mapped in $\sim 900$ hours of integration time. The strawman optical imager has a much smaller field-of-view and so would not be competitive for this type of survey.

- Targeted observations of the outskirts of galaxy clusters: PILOT could map the outer regions of clusters (or bright galaxies) where cosmic shear observations can discriminate between different dark matter halo profiles (see Hoekstra et al. 1998; Hoekstra, Yee, \& Gladders 2004). The viability of the cluster shear observational technique in infrared wavebands has recently been demonstrated by King et al. (2002).

- Targeted observations of superclusters: By mapping known superclusters, a cosmic shear survey can quantify the degree of filamentary structure present in the web of dark matter (see Gray et al. 2002), a critical observable discriminating between theories of structure formation.

PILOT could also make a valuable contribution in the regime of strong gravitational lensing, via observations of lensed arcs in the vicinity of high-redshift clusters. It has been shown that the probability of giant arc formation due to galaxy clusters is a sensitive measure of global cosmic parameters, particularly for the dark energy model (Bartelmann et al. 1998). Intriguingly, numerical simulations of clusters in the currently favoured cosmological constant model fall short by an order of magnitude in reproducing the observed abundance of large gravitational arcs (Bartelmann et al. 2003). However, another study suggests that simulations using realistic redshift distributions for background galaxies resolves this discrepancy in the observed arc statistics (Wambsganss, Bode, \& Ostriker 2004). Also, possible modifications to our current understanding of cluster sub-structure and evolution may be necessary to help resolve this difference in theory and observation.

A recent project addressing these issues is the 'RedSequence Cluster Survey', which mapped $90 \mathrm{deg}^{2}$ in Rand $z$-bands, discovering eight strong gravitational arcs (Gladders et al. 2003). Near-infrared imaging is a prerequisite for pursuing these studies to higher redshift. Hence we suggest another program addressing strong gravitational lensing:

- Survey for strongly lensed gravitational arcs in galaxy clusters: A $100 \mathrm{deg}^{2}$ survey to $\mathrm{K} \sim 21$ would yield $100-500$ clusters with velocity dispersions in excess of $700 \mathrm{~km} \mathrm{~s}^{-1}$ in the interval $1.0<z<1.5$. Doing this type of wide-field, near-IR 'arc survey' of galaxy clusters would address the above questions and obtain an 
accurate, independent measurement of cosmological parameters.

\section{An Observing Program for PILOT}

This paper has outlined an extensive range of challenging science projects that a telescope like PILOT could be used to tackle, from imaging of our planetary neighbours to searching for signatures from the first stars to form in the Universe. The projects also require a diverse range of instrumentation, from high resolution optical imagers using AO, through to sub-millimetre spectrometers. Some projects involve monitoring, making periodic measurements of a few hours duration every few weeks, while others are dedicated surveys that will require several months to accomplish. We anticipate that PILOT could be fully operational within three years of funding approval. While it is indeed possible that these projects could all be tackled with the same telescope, it is also unlikely that this would happen in practice. Indeed, given the low cost of an Antarctic 2-m telescope compared to substantially larger telescopes at temperate sites, it is quite possible that once PILOT is operating, with just one or two of the strawman instruments, the demand will be such that another similar-sized telescope would be built equipped with a different instrument complement, perhaps dedicated to a single project.

Nevertheless, it is still a useful exercise to examine the overall time requirements for the projects discussed in this document. These projects are summarized in Table 6 . Taken together, about three to four years of telescope time is needed to undertake them all. In practice, of course, as with all observing programs, this requires a detailed assessment of their individual needs, based on the final performance specifications achieved by the facility, and modified by the experience gained as the program is undertaken. Several programs, however, would take the lions

Table 6. Strawman Observing Program for PILOT

\begin{tabular}{|c|c|c|c|c|}
\hline Program & Cat. & Instrument & Type of observation & Time needed \\
\hline Orbital debris & $\mathrm{P}$ & $\begin{array}{l}\text { VRI WF } \\
\text { JHK WF }\end{array}$ & Monitor & 50 hours, twilight \\
\hline Planetary imaging & $\mathrm{P}$ & $\begin{array}{l}\text { VRI HR } \\
\text { JHK HR }\end{array}$ & Monitor & $\begin{array}{l}\text { Several days continuously } \\
\text { (Opp. Venus, Conj. Mars) }\end{array}$ \\
\hline Exo-planet transits & $\mathrm{P}$ & $\begin{array}{l}\text { VRI HR } \\
\text { JHK HR }\end{array}$ & Follow-up detections & $\begin{array}{l}10-20 \text { hours per candidate, } \\
\text { spread over } \sim 1 \text { week }\end{array}$ \\
\hline Disks & $\mathrm{P}$ & KLM & $\begin{array}{l}\text { Selected sources } \\
- \text { mini surveys }\end{array}$ & 100 hours \\
\hline Planetary microlensing & $\mathrm{P}$ & VRI HR & $\begin{array}{l}\text { Follow-up detections } \\
\text { - overrides }\end{array}$ & $\begin{array}{l}\text { 1-2 days continuous, } \\
\sim 6 \text { times per year }\end{array}$ \\
\hline Asteroseismology & $\mathrm{P}$ & VRI WF & $\begin{array}{l}\text { Monitor selected } \\
\text { sources }\end{array}$ & $\begin{array}{l}5-10 \text { days continuous, } \\
\text { once per year }\end{array}$ \\
\hline Massive Protostars & G & $\begin{array}{l}\text { NQ } \\
\text { Sub-mm }\end{array}$ & Selected sources & 50 hours \\
\hline Early SF census & G & Sub-mm & Selected sources & 50 hours \\
\hline Galactic ecology & G & KLM & Selected sources & 30 hours \\
\hline Molecular core Spect. & G & Sub-mm & Selected sources & 50 hours \\
\hline Brown Dwarfs & $\mathrm{P}+\mathrm{G}$ & KLM & Survey & 3 months \\
\hline Pulsar wind nebulae & G & VRI HR & Monitoring & Few minutes, weekly \\
\hline AGB Stars in LMC & G & KLM & Survey + Monitor & Few hours, weekly \\
\hline Stellar populations & G & $\begin{array}{l}\text { VRI WF } \\
\text { JHK HR }\end{array}$ & Survey & $\begin{array}{l}1 \text { month per field selected } \\
\text { (up to } 10 \text { fields) }\end{array}$ \\
\hline Stellar streams & G & $\begin{array}{l}\text { VRI WF } \\
\text { JHK HR }\end{array}$ & Survey & 1 month per field selected \\
\hline $\mathrm{SNe}$ in Starbursts & $\mathrm{C}$ & JHK HR & Monitor & 8 hours, 3 times per year \\
\hline Time delays in lenses & $\mathrm{C}$ & $\begin{array}{l}\text { VRI WF } \\
\text { JHK HR }\end{array}$ & Monitor & Daily images \\
\hline SF History Early Univ. & $\mathrm{C}$ & JHK WF & Survey & 50 hours \\
\hline Ultra deep field & $\mathrm{C}$ & JHK WF & Survey & 100 hours \\
\hline First light via GRBs & $\mathrm{C}$ & $\begin{array}{l}\text { JHK HR } \\
\text { KLM }\end{array}$ & Follow-up detections & 2 hours per override \\
\hline Cosmic shear & $\mathrm{C}$ & JHK WF & $\begin{array}{l}\text { Survey }+ \text { Selected } \\
\text { sources }\end{array}$ & 900 hours \\
\hline Strong lensing & $\mathrm{C}$ & JHK WF & Survey & 150 hours \\
\hline
\end{tabular}

Potential science programs for PILOT, as described in this paper (see Section 6), their instrument requirements and the approximate observing time they need. The programs are divided into the three major science categories $(\mathrm{P}=$ planetary, $\mathrm{G}=$ galaxy environment, $\mathrm{C}=$ cosmology) used here. The instruments are as described in Table 3 , with HR= high resolution and $\mathrm{WF}=$ wide field. The type of observation that each program requires is listed in the fourth column (e.g. survey, monitoring, follow-up from other observations, selected individual sources). An approximate time requirement to accomplish the program is given in the last column. 
share of the observing time. Surveys for old brown dwarfs, the determination of stellar populations in Local Group galaxies and in tidal streams, the ultra deep field in the near-IR searching for high-redshift galaxies, and mapping of the cosmic web of dark matter through gravitational lensing, each require several months to be devoted to them. A few programs need continuous monitoring, but only over short periods of time (e.g. orbital debris, planetary imaging, asteroseismology). Most of the monitoring programs could be simultaneously executed with the surveys by setting aside two to three hours each 'day' for their conduct (e.g. exo-planet transits, pulsar wind nebulae, SNe in starbursts, time delays in gravitational lenses). A few programs will need to make use of program overrides to be undertaken, most notably the follow up of GRB detections to search in the IR for signatures of the First Light at extremely high redshift, and to monitor planetary microlensing candidates. Programs which require mid-IR and sub-millimetre instrumentation, which are generally the most complex to operate in view of their cryogenic requirements, can however be conducted during daylight and so could be undertaken during the summer period. This particularly applies to studies of the early stages of star formation and the chemical environment of molecular cores. In addition, monitoring of global atmospheric changes in Mars and Venus also can be conducted during daylight hours, particularly in twilight. Characterizing orbital debris is also best undertaken in the twilight period. Finally, there are a number of smaller projects, generally studying selected sources at thermal-IR wavelengths, that would require smaller time allocations (e.g. searches for proto-planetary disks, studying the Galactic ecology, signatures of the early stages of massive star formation).

\section{Beyond PILOT: ELTs and Interferometers}

PILOT would be capable of a wide range of science but, as its name suggests, the telescope is primarily envisaged as a pathfinder for more powerful facilities to follow, able to fully exploit the most advantageous conditions available for ground-based astronomy on earth. PILOT may lead to larger telescopes, from the size of the current 8-m class telescopes to the era of Extremely Large Telescopes (ELTs), with apertures of $20 \mathrm{~m}$ or more. As well, PILOT may provide a path to networks of telescopes, as envisaged with the proposed KEOPS (Vakili et al. 2004) and Antarctic Planet Interferometer (API; Swain et al. 2003), several 2-4-m class telescopes placed over baselines of up to one kilometre at Dome $\mathrm{C}$ and operating in the thermalIR, and the API's own pathfinder facility, the API Science Demonstrator (API-SD; Coudé du Foresto et al. 2003). We briefly consider here what such facilities might be able to accomplish.

As indicated by the performance calculations in Section 4.1.1, an 8-m telescope at Dome $\mathrm{C}$ would be more sensitive than any temperate-latitude 8 -m telescope, by between 1 and $3 \mathrm{mag}$, depending on the part of the optical and infrared bands being compared. It would also provide superior spatial resolution on account of the better seeing, and a wider wavelength coverage through opening windows in the mid-IR. The performance of an Antarctic $8 \mathrm{~m}$ can also be compared to that of a temperate latitude ELT, in much the same way that we compared the performance of the 2-m PILOT to a temperate 8-m telescope in Section 4.1.1. For instance, the sensitivities of the Antarctic 8-m and a temperate 30-m ELT would typically differ by less than a magnitude (cf. Tables 1 and 2), and the same advantages of wavelength coverage and good seeing would apply to the Antarctic telescope as before. Adaptive optics is critical to the future success of ELTs, for their science drivers require not just extraordinary sensitivity, but superlative spatial resolution as well. As discussed in Section 4.1.2, the good seeing, wider isoplanatic angle and longer coherence times at Dome $\mathrm{C}$ all serve to make $\mathrm{AO}$ operation there significantly easier than at temperate locations, for the same level of performance.

The science cases for these grand design facilities are predicated on two major objectives. The first is to conduct ultra deep field surveys in the infrared, able to probe back through the entire history of the Universe to the epoch of CMBR formation, in particular to the time when the first stars formed. The second is the detection of exo-earths, planets like our own orbiting other stars.

The sensitivity of a telescope operating in background limited conditions at the diffraction limit, $S_{\lambda}$, is proportional to $D^{2} \sqrt{B / t}$, where $D$ is the telescope diameter, $B$ the background flux and $t$ the integration time. A figure of merit of its power to survey a field of solid angle $\Omega$ (larger than the field of view of the telescope) in total time $T$ is thus inversely proportional to $S_{\lambda}$ and given by $D^{2} \sqrt{T \Omega / B}$ (see also Angel 2005). Two performance comparisons of relevance to the discussion may be made here.

The first is when comparing an Antarctic 8-m to a temperate ELT. Whereas the background reduction is equivalent to needing a telescope of half the diameter to complete the survey in the same time, it is also notable that it is significantly easier to provide a wide field of view on the smaller facility, and this will be reflected through the increased cost of instrumentation for the larger facility.

The second comparison is with future planned space facilities, such as the 6-m JWST (see Angel, Lawrence, \& Storey 2004). At shorter infrared wavelengths, where the further background reduction from Antarctica to a spacebased location is less than two orders of magnitude, a $20 \mathrm{~m}$ at Dome $\mathrm{C}$ will be competitive to the JWST for spectroscopic applications for $\lambda<4 \mu \mathrm{m}$. It will also provide far easier access for the necessarily sophisticated instrumentation requirements for spectroscopy. Such follow-up of ultra deep field sources is an essential aspect of interpreting the data that surveys from the JWST will produce.

The task of detecting exo-earths is even more formidable. For a sun-like star $10 \mathrm{pc}$ from the Earth, for which there are about a dozen, an exo-earth would be separated from its parent star by $0.1^{\prime \prime}$. It would be $\sim 10^{-10}$ times as bright in the visible, rising to a maximum contrast of $\sim 10^{-6}$ times around $20 \mu \mathrm{m}$. It would also induce a 
wobble in the star's position of about 1 micro-arcsec with a period of 1 year. Detecting the signature of the planet flux from within the point spread function of the star is the challenge for ELTs. A crucial aspect of being able to do so is extremely good correction of the atmospheric turbulence in order to minimize the size of the point spread function (including any scattered light) of the AO system. The technology to accomplish such a feat has yet to be developed, but it is possible to calculate the performance of prospective systems. By virtue of the superior conditions for AO correction at Dome $\mathrm{C}$, a telescope capable of detecting exo-earths can have smaller size if built there than at a temperate site. Indicative calculations by Lardière et al. (2004), for example, suggest that a 15-m telescope at Dome $\mathrm{C}$ might suffice for such a detection in the visible and near-IR, whereas a 30-m telescope would be needed from Mauna Kea.

While ELTs will provide superb sensitivity, many of the most topical astrophysical research frontiers entail observation of matter in the close environment of stars or in highly luminous cores - i.e they require exquisite spatial resolution to conduct. Studies of exo-planets, star formation, stellar winds, and active galactic nuclei are all limited by the extreme dynamic ranges, high angular resolutions, and high measurement precision needed to discriminate the faint signals against the glare of the central star. These considerations drive optical designs towards an interferometer; however, at mid-latitude sites there is a heavy penalty caused by the seeing in the turbulent atmosphere. Quantifying just how strong this penalty will be depends in detail on the experiment; however, interferometer performance will in general be highly sensitive to all three fundamental seeing parameters: $r_{0}$ (spatial), $t_{0}$ (temporal), and $\theta_{0}$ (angular) coherence properties of the incoming wavefront. From the Antarctic plateau, all three of these atmospheric properties attain their most favorable values on the surface of the earth, as described in Section 2.

For techniques such as nulling interferometry and astrometric interferometry, the improved atmospheric conditions should result in orders of magnitude increase in sensitivity on top of gains already available from the extreme low temperature and water vapour. For instance, as discussed in Section 2, the mean square astrometric error of a dual-beam interferometer depends on the strength of the turbulence, weighted by the square of the height at which that turbulence occurs. At temperate sites turbulence sets a limits of around 100 micro-arcseconds in the accuracy to which positions can be measured (Shao \& Colavita 1992). While this may be sufficient to detect Jupiter-type systems, it is two orders of magnitude away from the precision needed to detect exo-earths. The necessary precision is within the range of an instrument operating at Dome C (Lloyd et al. 2002).

Imaging nulling interferometers may not only be able to detect exo-earths, but be able to analyze their atmospheres. This would permit, for example, the signature of nonequilibrium chemistry, that is readily apparent in the earth's atmosphere and induced by the presence of biota in the environment (as indicated by the simultaneous presence of $\mathrm{CO}_{2}, \mathrm{H}_{2} \mathrm{O}$, and $\mathrm{O}_{3}$ in the mid-IR spectral window; see Angel \& Woolf 1997), to be detected.

The potential for Antarctic interferometry is fundamentally different from that at a conventional site. The proposed Antarctic Plateau Interferometer could be operating before the immensely more expensive space missions, and so begin the task of characterizing exo-planets and searching for objects down to Earth masses (see also Storey et al. 2002; Storey 2004). Furthermore, a wealth of other stellar astrophysics could be addressed using such a device. Observations of disks around young stellar objects should reveal substructure such as spiral density waves and gap clearing due to planet growth. Mass loss phenomena in evolved stars, and substructure such as disks and jets within dusty compact micro-quasars, will be within reach of imaging observations. Studies of fundamental stellar properties - sizes, effective temperatures, distances, and (using binary stars) masses - will mean that almost every branch of stellar astronomy will benefit.

The roadmap towards the construction of a fullycapable Antarctic interferometer is being articulated through the proposal to build the API-SD. The first phase aims to ensure that the required infrastructure for an interferometer is constructed at Dome $\mathrm{C}$. In the second phase, the modest initial 2-element $40 \mathrm{~cm}$ optics of the Science Demonstrator would be upgraded to a number (up to six) 2-4-m sized telescopes. PILOT would have direct and immediate utility to the API project. It could be used as one array element on a part-time basis for dedicated projects, or used to perform initial sky-testing and sensitivity measurements before the upgrade path to larger apertures is finalized. Furthermore, PILOT and API have many common requirements apart from being winter-fast 2-m telescopes. Nulling interferometry, for example, will require delivery of high and stable Strehl ratios from the adaptive optics system, a feature which can be verified with PILOT.

\section{Conclusions}

Modest-sized telescopes, built for optical and infrared imaging and placed at Dome $\mathrm{C}$, would be able to perform a wide range of competitive science for a fraction of the cost incurred by larger facilities that are built at temperate locations. Wide-field infrared imaging surveys, particularly in the K-, L-, and M-bands (from 2.3 to $5 \mu \mathrm{m}$ ), conducted with near-diffraction limited resolution, provide the greatest performance gains for Antarctic telescopes. Near-diffraction limited imaging could also be obtained at optical wavelengths with a 2-m sized telescope, a regime not attempted from temperate sites because of their adverse site seeing characteristics. An Antarctic telescope can also explore the cosmos through new ground-based windows, from 17 to $40 \mu \mathrm{m}$ and at $200 \mu \mathrm{m}$.

The performance characteristics we describe for such an Antarctic telescope assist science projects aimed at studying planetary systems, star formation within the 
Milky Way, the formation and evolution of the galaxies in our Local Group, the star formation history of the Universe from the time of formation of the first massive stars, and the structure of the cosmic web of dark matter.

PILOT, the Pathfinder for an International Large Optical Telescope, the 2-m sized telescope described in this paper as a facility able to tackle this diverse range of science, is, however, only designed as a pathfinder. Antarctica offers an opportunity to pursue projects that would require either much larger facilities if they were sited elsewhere, or need considerably more expensive space facilities. Before such projects can be contemplated, it has to be first demonstrated that it is indeed possible to operate the complex instrumentation needed for the science from Antarctica. PILOT's principal aim is to do this, by showing that the special conditions of the Antarctic plateau can indeed be utilized for the conduct of sophisticated experiments. PILOT may indeed serve as a prototype towards the construction of large optical and infrared telescopes in Antarctica, even the so-called ELTs. However PILOT will equally well serve as a technology demonstrator towards the construction of infrared interferometers, operating with kilometre-sized baselines. Both these kinds of facilities would be able to further two of the grand design astrophysical projects being pursued today: The imaging of ultra deep fields to uncover the complete star formation history of the Universe from the time of the First Light, and the search for, and subsequent characterization of, other earth-like planets in the Galaxy.

\section{Acknowledgements}

Many of our colleagues have contributed to this paper through vigorous scientific discussions, and we wish to acknowledge them for the ideas this has help generated. Our thanks go to David Bennett, Paolo Calisse, Jessie Christiansen, Jon Everett, Suzanne Kenyon, Will Saunders, and Peter Wood. We also thank the Australian Research Council for funding support through the Discovery Grant and Australian Postdoctoral Fellowship programs, the Australian Antarctic Division for a postgraduate scholarship, and the University of New South Wales for its continued support of the Antarctic astronomy program. We also thank the referee, Gerry Gilmore, for his helpful comments on the paper.

\section{References}

Abe, F., et al. 2004, Sci, 305, 1264

André, P., Motte, F., \& Bacmann, A. 1999, ApJL, 513, L57

Angel, J. R. P., \& Woolf, N. J. 1997, ApJ, 475, 373

Angel, R. 2005, Proc. SPIE, 5487, 1

Angel, R., Lawrence, J., \& Storey, J. 2004, Proc. SPIE, 5382, 76

Aristidi, E., et al. 2005, A\&A, 430, 739

Aristidi, E., Agabi, K., Vernin, J., Azouit, M., Martin, F., Ziad, A., \& Fossat, E. 2003, A\&A, 406, L19

Ashley, M. C. B., Burton, M. G., Calisse, P. G., Phillips, A., \& Storey, J. W. V. 2005, in IAU Pub. Highlights in Astronomy, 13, eds. O. Engvold, \& M. G. Burton (Ast. Soc. Pac.), in press
Ashley, M. C. B., Burton, M. G., Lawrence, J. S., \& Storey, J. W. V. 2004, AN, 325, 619

Ashley, M. C. B., Burton, M. G., Storey, J. W. V., Lloyd, J. P., Bally, J., Briggs, J. W., \& Harper, D. A. 1996, PASP, 108, 721

Bacon, D. J., Massey, R. J., Refregier, A. R., \& Ellis, R. S. 2003, MNRAS, 344, 673

Bailey, J., Chamberlain, S., Walter, M., \& Crisp, D. 2004, in Proc. 3rd European Workshop in Exo-Astrobiology, ESA-SP 545, 7, eds. A. Harris, \& L. Ouwehand (Noordwijk: ESA Publications)

Baldwin, J. E., Tubbs, R. N., Cox, G. C., Mackay, C. D., Wilson, R. W., \& Andersen, M. I. 2001, A\&A, 368, L1

Bartelmann, M., Huss, A., Colberg, J. M., Jenkins, A., \& Pearce, F. R. 1998, A\&A, 330, 1

Bartelmann, M., Meneghetti, M., Perrotta, F., Baccigalupi, C., \& Moscardini, L. 2003, A\&A, 409, 449

Bedding, T. R., \& Kjeldsen, H. 2003, PASA, 20, 203

Benjamin, R. A., et al. 2003, PASP, 115, 953

Bennett, D. P., et al. 2004, Proc. SPIE, 5487, 145

Bergin, E. A., Alves, J., Huard, T., \& Lada, C. J. 2002, ApJ, 570, L101

Bond, I. A., et al. 2002, MNRAS, 333, 71

Bond, I. A., et al. 2004, ApJ, 606, L155

Bonnell, I. A., \& Bate, M. R. 2002, MNRAS, 336, 659

Brown, T. M. 2003, ApJ, 593, L125

Brown, T. M., Ferguson, H. C., Smith, E., Kimble, R. A., Sweigart, A. V., Renzini, A., Rich, R., \& VandenBerg, D. A. 2003, ApJ, 592, L17

Burrows, A., Hubbard, W. B., Lunine, J. I., \& Liebert, J. 2001, RvMP, 73, 719

Burton, M. G., Storey, J. W. V., \& Ashley, M. C. B. 2001, PASA, 18,158

Burton, M. G., et al. 1994, PASA, 11, 127

Burton, M. G., et al. 2000, ApJ, 542, 359

Caldwell, D. A., Borucki, W. J., Showen, R. L., Jenkins, J. M., Doyle, L., Ninkov, Z., \& Ashley, M. 2004, in Bioastronomy 2002: Life Among the Stars, Proc. IAU Symp. 213, 93, eds. R. Norris, \& F. Stootman (San Fransisco, CA: ASP)

Calisse, P. G., Ashley, M. C. B., Burton, M. G., Phillips, M. A., Storey, J. W. V., Radford, S. J. E., \& Peterson, J. B. 2004, PASA, 21,256

Candidi, M., \& Lori, A. 2003, MmSAI, 74, 29

Cen, R. 2003, ApJ, 591, 12

Chamberlain, M. A., Ashley, M. C. B., Burton, M. G., Phillips, M. A., \& Storey, J. W. V. 2000, ApJ, 535, 501

Chamberlin, R. A., Lane, A. P., \& Stark, A. A. 1997, ApJ, 476, 428

Chamberlin, R. A., Martin, R. N., Martin, C. L., \& Stark, A. A. 2003, Proc. SPIE, 4855, 9

Chanover, N. J., Glenar, D. A., \& Hillman, J. J. 1998, JGR, 103, 31355

Christensen-Dalsgaard, J. 2002, RvMP, 74, 1073

Clough, S. A., \& Iacono, M. J. 1995, JGR, 100, 16519

Coudé du Foresto, V., Swain, M., Schneider, J., \& Allard, F. 2003, MSAIS, 2, 212

Dantowitz, R. F., Teare, S. W., \& Kozubal, M. J. 2000, AJ, 119, 2455

Dempsey, J. T., Storey, J. W. V., \& Phillips, M. A. 2005, PASA, 22, 91

Dickinson, M., et al. 2000, ApJ, 531, 624

Doherty, M., et al. 2004, MNRAS, 354, 7

Drake, A. J., \& Cook, K. H. 2004, ApJ, 604, 379

Egan, M. P., van Dyk, S. D., \& Price, S. D. 2001, AJ, 122, 1844

Erb, D. P., Shapley, A. E., Steidel, C. C., Pettini, M., Adelberger, K. L., Hunt, M. P., Moorwood, A. F. M., \& Cuby, J. 2003, ApJ, 591, 101

Ferguson, A. M. N., Irwin, M. J., Ibata, R. A., Lewis, G. F., \& Tanvir, N. R. 2002, AJ, 124, 1452

Forget, F., Hourdin, F., Fournier, R., Hourdin, C., Talagrand, O., Collins, M., Lewis, S. R., Read, P. L., \& Huot, J.-P. 1999, JGRE, 104,24155

Fossat, E. 2003, MSAIS, 2, 139

Fowler, A. M., et al. 1998, Proc. SPIE, 3354, 1170 
Franx, M., et al. 2003, ApJ, 587, L79

Freedman, W. L., et al. 2001, ApJ, 533, 47

Freeman, K., \& Bland-Hawthorn, J. 2002, ARAA, 40, 487

Gibb, E. L., et al. 2000, ApJ, 536, 347

Gladders, M. D., Hoekstra, H., Yee, H. K. C., Hall, P. B., \& Barrientos, L. F. 2003, ApJ, 593, 48

Glazebrook, K., et al. 2004, Natur, 430, 181

Glazebrook, K., Blake, C., Economou, F., Lilly, S., \& Colless, M. 1999, MNRAS, 306, 843

Gould, A., \& Loeb, A. 1992, ApJ, 396, 104

Gray, M. E., Taylor, A. N., Meisenheimer, K., Dye, S., Wolf, C., \& Thommes, E. 2002, ApJ, 568, 141

Griest, K., \& Safizadeh, N. 1998, ApJ, 500, 37

Hereld, M. 1994, in Astrophys., \& Sp. Sci. Lib., 190, Infrared Astronomy with Arrays, the Next Generation, ed. I. McLean (Dordrecht: Kluwer), 248

Hester, J. J., et al. 2002, ApJ, 577, L49

Hidas, M. G., Burton, M. G., Chamberlain, M. A., \& Storey, J. W. V. 2000, PASA, 17, 260

Hidas, M. G., et al. 2005, MNRAS, 360, 703

Hoekstra, H., Franx, M., Kuijken, K., \& Squires, G. 1998, ApJ, 504,636

Hoekstra, H., Yee, H. K. C., \& Gladders, M. D. 2002, NewAR, 46, 767

Hoekstra, H., Yee, H. K. C., \& Gladders, M. D. 2004, ApJ, 606, 67

Hopkins, A. M., Connolly, A. J., \& Szalay, A. S. 2000, AJ, 120, 2843

Ibata, R., Irwin, M., Lewis, G., Ferguson, A. M. N., \& Tanvir, N. 2001, Natur, 412, 49

Ibata, R., Chapman, S., Ferguson, A. M. N., Irwin, M., Lewis, G., \& McConnachie, A. W. 2004, MNRAS, 351, 117

Indermuehle, B. T., Burton, M. G., \& Maddison, S. T. 2005, PASA, 22,73

Ivezić, Z., \& Elitzur, M. 1997, MNRAS, 287, 799

Juneau, S., et al. 2005, ApJL, 619, 135

Kaiser, N., \& Squires, G. 1993, ApJ, 404, 441

Kaiser, N., Squires, G., \& Broadhurst, T. 1995, ApJ, 449, 460

King, L. J., Clowe, D. I., Lidman, C., Schneider, P., Erben, T., Kneib, J.-P., \& Meylan, G. 2002, A\&A, 385, 5

Kirkpatrick, J. D., Allard, F., Bida, T., Zuckerman, B., Becklin, E. E. Chabrier, G., \& Baraffe, I. 1999, ApJ, 519, 802

Kjeldsen, H., \& Frandsen, S. 1992, PASP, 104, 413

Kochanek, C. 2002, ApJ, 578, 25

Kochanek, C., \& Schechter, P. 2004, in Measuring and Modeling the Universe, Carnegie Ob. Ast. Ser., ed. W. L. Freedman (Cambridge: Cambridge University Press), 117

Lada, C. 1999a, in NATO ASIC Proc. 540, The Origin of Stars and Planetary Systems, 143 (Dordrecht: Kluwer)

Lada, E. 1999b, in NATO ASIC Proc. 540, The Origin of Stars and Planetary Systems, 441 (Dordrecht: Kluwer)

Lamb, D. Q., \& Reichart, D. E. 2001, Proc. 20th Texas Symp. on Relativistic Astrophysics, AIPC, 586, 605

Lane, A. P. 1998, in ASP Conf. Proc., Vol. 141, eds. G. Novak, \& R. H. Landsberg, 289 (San Fransisco, CA: ASP)

Lane, A. P., \& Stark, A. A. 1997, Ant. J. US, 30, 377

Lardière, O., Salinari, P., Jolissaint, L., Carbillet, M., Riccardi, A., \& Esposito, S. 2004, Proc. SPIE, 5382, 550

Lawrence, J. 2004a, PASP, 116, 482

Lawrence, J. 2004b, ApOpt, 43, 1435

Lawrence, J. S., Ashley, M. C. B., Tokovinin, A., \& Travouillon, T. 2004, Natur, 431, 278

Lewis, G. F., \& Belle, K. E. 1998, MNRAS, 297, 69

Lewis, G. F., Irwin, M. J., Hewett, P. C., \& Foltz, C. B. 1998, MNRAS, 295, 573

Lilly, S. J., Le Fevre, O., Hammer, F., \& Crampton, D. 1996, ApJ, 460, L1

Lloyd, J. P., Oppenheimer, B. R., \& Graham, J. R. 2002, PASA, 19,318

Longmore, S. N., Burton, M. G., Minier, V., \& Walsh, A. J. 2005, MNRAS, submitted
Madau, P., Ferguson, H. C., Dickinson, M. E., Giavalisco, M., Steidel, C. C., \& Fruchter, A. 1996, MNRAS, 283, 1388

Maercker, M., Burton, M. G., \& Wright, C. 2005, A\&A, submitted

Mao, S., \& Paczynski, B. 1991, ApJ, 374, L37

Marcy, G. W., \& Butler, R. P. 1998, ARAA, 36, 57

Marks, R. D. 2002, A\&A, 385, 328

Marks, R. D., Vernin, J., Azouit, M., Briggs, J. W., Burton, M. G., Ashley, M. C. B., \& Manigault, J. F. 1996, A\&AS, 118,385

Marks, R. D., Vernin, J., Azouit, M., Manigault, J. F., \& Clevelin, C. 1999, A\&AS, 134, 161

Martin, C. L., Walsh, W. M., Xiao, K., Lane, A. P., Walker, C. K., \& Stark, A. A. 2004, ApJS, 150, 239

Mattila, S., \& Meikle, W. P. S. 2001, MNRAS, 324, 325

McConnachie, A. W., Irwin, M. J., Lewis, G. F., Ibata, R. A., Chapman, S. C., Ferguson, A. M. N., \& Tanvir, N. R. 2004, MNRAS, 351, 94

McKee, C. F., \& Tan, J. C. 2002, Natur, 416, 59

Mediavilla, E., et al. 1998, ApJ, 503, 27

Melatos, A., et al. 2005, ApJ, in press

Minier, V., Burton, M. G., Hill, T., Pestalozzi, M. R., Purcell, C., Longmore, S., Garay, G., \& Walsh, A. 2005, A\&A, 429, 945

Motta, V., Mediavilla, E., Munoz, J. A., \& Falco, E. 2004, ApJ, 613,86

Nguyen, H. T., Rauscher, B. J., Severson, S. A., Hereld, M., Harper, D. A., Lowenstein, R. F., Morozek, F., \& Pernic, R. J. 1996, PASP, 108, 718

Oppenheimer, B. R., Kulkarni, S. R., Matthews, K., \& van Kerkwijk, M. H. 1995, Sci, 270, 1478

Paczynski, B. 1986, ApJ, 304, 1

Payne, J. M. 2002, in ASP Conf. Ser., 278. Single-Dish Radio Astronomy: Techniques and Applications, 453 (San Fransisco, CA: ASP)

Phillips, M. A., Burton, M. G., Ashley, M. C. B., Storey, J. W. V., Lloyd, J. P., Harper, D. A., \& Bally, J. 1999, ApJ, 527, 1009

Pojmanski, G. 2002, AcA, 52, 397

Ragazzoni, R., et al. 2004, Proc. SPIE, 5489, 481

Refregier, A. R. 2003, ARAA, 41, 645

Rhie, S. H., et al. 2000, ApJ, 533, 378

Rhoads, J. E., et al. 2004, ApJ, 611, 59

Ruhl, J., et al. 2004, Proc. SPIE, 5498, 11

Ryder, S. D., Sadler, E. M., Subrahmanyan, R., Weiler, K. W., Panagia, N., \& Stockdale, C. 2004, MNRAS, 349, 1093

Shao, M., \& Colavita, M. M. 1992, A\&A, 262, 353

Staguhn, J. G., et al. 2003, Proc. SPIE, 4855, 100

Stark, A. A. 2002, AIP Conf. Proc., 616, Experimental Cosmology at Millimetre Wavelengths, 83

Stark, A. A. 2003, The Future of Small Telescopes in The New Millennium, Vol. II: The Telescopes We Use, 269 (Dordrecht: Kluwer)

Stark, A. A., Bolatto, A. D., Chamberlin, R. A., Lane, A. P., Bania, T. M., Jackson, J. M., \& Lo, K.-Y. 1997, ApJ, 480, L59

Steidel, C. C., Adelberger, K. L., Giavalisco, M., Dickinson, M., \& Pettini, M. 1999, ApJ, 519, 1

Storey, J. W. V. 2004, Proc. SPIE, 5491, 169

Storey, J. W. V., Ashley, M. C. B., Lawrence, J. S., \& Burton, M. G. 2003, Mem. Soc. Ast. It., 2, 13

Storey, J. W. V., Burton, M. G., \& Ashley, M. C. B. 2002, Proc. SPIE, 4835,110

Swain, M. R., Coudé du Foresto, V., Fossat, E., \& Vakili, F. 2003, MSAIS, 2, 207

Sudbury Neutrino Observatory Collaboration, 2002, PRvL, 89, 011301

Swinbank, A. M., et al. 2005, MNRAS, 359, 401

Tingley, B. 2004, A\&A, 425, 1125

Travouillon, T., Ashley, M. C. B., Burton, M. G., Storey, J. W. V., \& Loewenstein, R. F. 2003a, A\&A, 400, 1163 
Travouillon, T., et al. 2003b, A\&A, 409, 1169

Vakili, F., et al. 2004, Proc. SPIE, 5491, 1580

van Dishoeck, E. F. 2004, ARAA, 42, 119

van Loon, J. Th. 2000, A\&A, 354, 125

Walden, V. P., Town, M. S., Halter, B., \& Storey, J. W. V. 2005, PASP, 117,300

Wambsganss, J., Bode, P., \& Ostriker, J. P. 2004, ApJ, 606, 93

Ward-Thompson, D., André, P., \& Kirk, J. M. 2002, MNRAS, 329,257
Wayth, R. B., Warren, S. J., Lewis, G. F., \& Hewett, P. C. 2005 , MNRAS, 360, 1333

Wood, P. R. 1998, A\&A, 338, 592

Yan, L., McCarthy, P. J., Freudling, W., Teplitz, H. I., Malumuth, E. M., Weymann, R. J., \& Malkan, M. A. 1999, ApJ, 519, L47

Yan, H., \& Windhorst, R. A. 2004, ApJ, 612, 93 